\title{
Radiative Feedback from Massive Stars: Ionizing Radiation and Its Fate in Starburst Galaxies
}

\author{
by \\ Jordan Alexandra Zastrow \\ A dissertation submitted in partial fulfillment \\ of the requirements for the degree of \\ Doctor of Philosophy \\ (Astronomy and Astrophysics) \\ in The University of Michigan \\ 2013
}

Doctoral Committee:

Associate Professor Sally Oey, Chair

Professor Lee William Hartmann

Professor Timothy A. McKay

Professor Sylvain Veilleux, University of Maryland 
Copyright (C) Jordan Alexandra Zastrow 2013

All Rights Reserved 
To my parents, for giving me the freedom and love to chase my dreams 


\section{ACKNOWLEDGMENTS}

I give my deepest gratitude to Sally Oey; I couldn't have asked for a more incredible mentor during my years at Michigan. Thank you for your patience, for the observing opportunities, and for giving me the independence to make mistakes and learn from them along the way. I could't have done this without your valuable advice and support. Thank you for showing by example effective ways to teach, both on a classroom level and as a mentor.

I have been lucky to work with many incredible astronomers over the years. To Eric Pellegrini, thank you for the many hours you willingly spent discussing research and problem solving with me. Thank you to Colin Slater for help with observations and for answering my numerous python questions. Thank you to Sylvain Veilleux and Michael McDonald for their help reducing and analyzing MMTF data, and to Crystal Martin for helpful discussions. Thanks to the FANG research group who have helped me become a better scientist through group discussion of weekly arXiv posts, mock TAC meetings, research feedback and by providing a sounding board for ideas. Among these members are: Sally Oey, Eric Pellegrini, Anne Jaskot, Joel Lamb, Carolina Kehrig, Dan Hanish, Andrew Graus, and Dominique Segura-Cox. I want to particularly thank Joel Lamb and Anne Jaskot, my fellow writing group members, who have helped me develop my written communication and composition

skills. I especially thank the members of my thesis committee, Lee Hartmann, Sylvain Veilleux, and Timothy McKay, for their helpful comments and feedback on my thesis work.

I am grateful for the support that I have received from the University of Michigan Astronomy department. I thank our administrative staff, in particular Brian Cox, for making the wheels of the department turn and for answering my numerous questions 
and requests; I thank Shannon Murphy for help teaching and in the planetarium. I additionally want to thank Nuria Calvet for always being willing to offer advice and support, even after the end of her term as graduate-student advisor.

And of course, I thank my fellow grad students, without whom my time at Michigan would have been much poorer. In particular, I thank Melissa McClure, my officemate and friend of five years, for putting up with my cluttered office habits; to Laura Ingleby, Jess Werk, Shannon Schmoll, and Jen Blum, thanks for taking me under your 'wings' and showing me the ins and outs of life in Ann Arbor. A big thanks to Ashley King, my dear friend and fellow triathlete, for being my partner-in-crime on our many Ann Arbor adventures.

I would like to give a special thanks to Tony Diaz Tushman for standing by me all these years. Thank you for always being there to offer emotional support, the occasional distraction, and for giving me the courage to stand up for the things that I need and want.

To my parents, Jon Zastrow and Rachael Rice, I offer my sincere appreciation and gratitude. You have always believed that I could accomplish anything that I desired. Your unconditional love and support has helped me through the challenging, yet rewarding, experience of graduate school.

Chapter 2 of this work is published in The Astrophysical Journal Letters, Vol 741, page L17 (Zastrow et al., 2011), and is reproduced here with permission and minor modifications. It was supported by NSF grants AST-0806476 to M.S. Oey and AST-1009583 to Sylvain Veilleux. Chapter 3 of this work has been submitted to be published in The Astrophysical Journal (Zastrow et al., submitted) and is reproduced here with permission. This work was supported by NSF grants AST0806476, AST-0907758, and AST-1210285 and a Margaret and Herman Sokol Faculty award to M.S. Oey. This work was also funded by NSF grants AST-0606932 and AST1009538 to Sylvain Veilleux and Michael McDonald, as well as a Hubble Fellowship grant HST-HF51308.01-A awarded to Michael McDonald by the Space Telescope Science Institute, which is operated by the Association of Universities for Research in Astronomy, Inc., for NASA, under contract NAS 5-26555. Chapter 4 of this work is 
published in The Astrophysical Journal Vol 769, page 94 (Zastrow et al., 2013), and is reproduced here with permission and minor modifications. It was also supported by AST-0806476 and AST-0907758. In addition, I appreciate the numerous travel grants given by the Rackham Graduate School to support presenting my work at conferences. 


\section{CONTENTS}

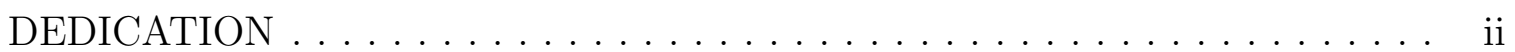

ACKNOWLEDGEMENTS ....................... iii

LIST OF FIGURES . . . . . . . . . . . . . . . . . . . viii

LIST OF TABLES . . . . . . . . . . . . . . . . .

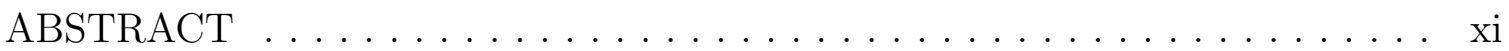

CHAPTER

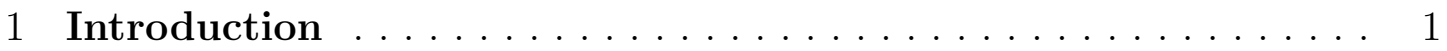

2 An Ionization Cone in NGC $5253 \ldots \ldots \ldots \ldots$

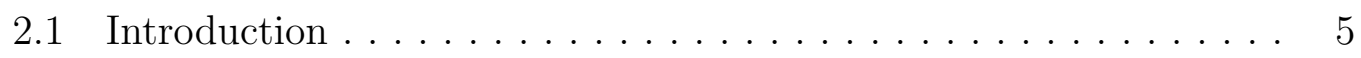

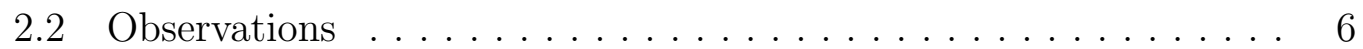

2.3 Ionization Cone in NGC $5253 \ldots \ldots \ldots \ldots \ldots$

2.3.1 Morphology and Excitation of the Ionization Cone . . . 8

2.3 .2 Kinematics . . . . . . . . . . . . . . . . 12

2.3 .3 Possible AGN . . . . . . . . . . . . . . . . . . . . 14

2.4 Implications: Orientation Bias . . . . . . . . . . . . . . . 15

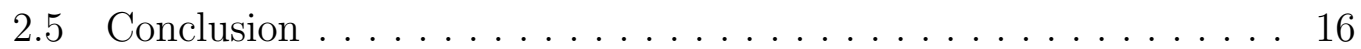

3 New Constraints on the Escape Fraction of Ionizing Photons from Local Starbursts Using [SIII]/[SII] Ionization Parameter

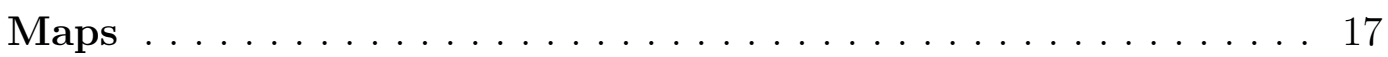

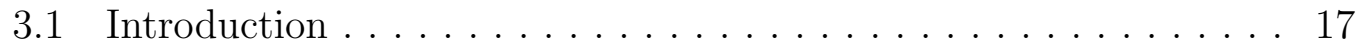

3.2 Method and Data . . . . . . . . . . . . . . . . . . . 19

3.2 .1 Method........................ 19

3.2 .2 Data........................... 20 


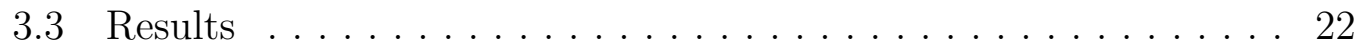

3.3.1 Galaxies with Extended emission . . . . . . . . . . . 22

3.3 .2 Non-Detections . . . . . . . . . . . . . . . . . . . 36

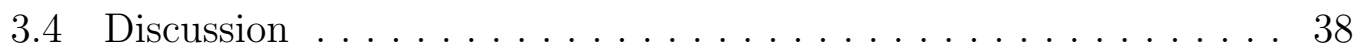

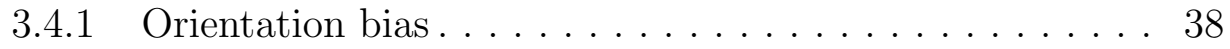

3.4.2 Possible Trends with Galaxy Properties . . . . . . . . . . 40

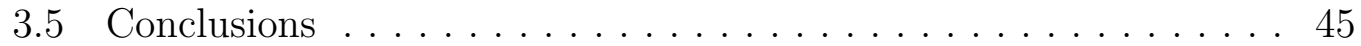

4 Single-Star HII Regions as a Probe of Massive Star SEDs . . . . 50

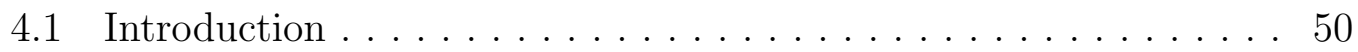

4.1.1 Description of the Model Atmosphere Codes . . . . . . . 52

4.2 Observations and Method .................. 54

4.2 .1 Observations . . . . . . . . . . . . . . . . . . . . 54

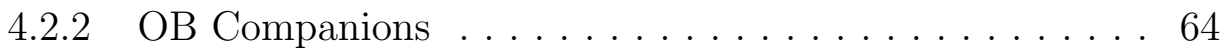

4.2 .3 Method.......................... 65

4.3 Photoionization Models . . . . . . . . . . . . . . . . . 70

4.3.1 Uniform Density Models . . . . . . . . . . . . . . . 70

4.3 .2 Density Clumps . . . . . . . . . . . . . . . . . . 73

4.3.3 Rate of Ionizing Photons _ . . . . . . . . . . . . 78

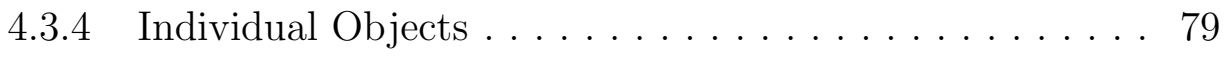

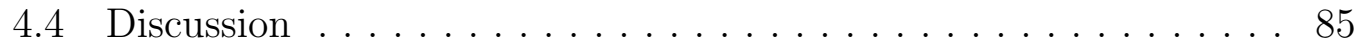

4.4.1 Comparison of Different Atmosphere Models . . . . . . 85

4.4.2 Calibration between $\mathrm{SpT}$ and $T_{\text {eff }} \ldots \ldots \ldots \ldots . \ldots 7$

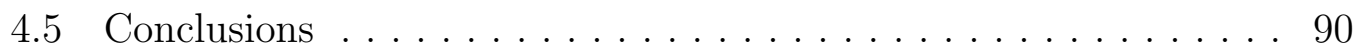

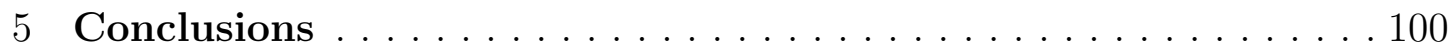

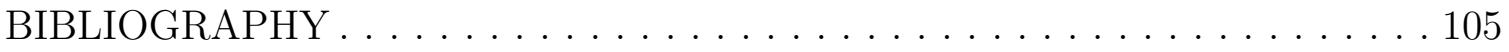




\section{LIST OF FIGURES}

Figure

2.1 Composite image of NGC $5253 \ldots \ldots \ldots \ldots \ldots \ldots \ldots$

$2.2[\mathrm{SIII}] /[\mathrm{SII}]$ ratio map for NGC $5253 \ldots \ldots \ldots \ldots \ldots \ldots$

2.3 Emission-line ratios vs. galactocentric radius. . . . . . . . . . . . . 11

2.4 NGC $5253 \mathrm{H} \alpha$ image, minor axis oriented along the $y$-axis. . . . . . . 13

3.1 Emission-line images of NGC 3125. . . . . . . . . . . . . . . . . 24

3.2 Line ratios as a function of height above and below the major axis for the ionization cone (red points) and the mean line ratio from rest of

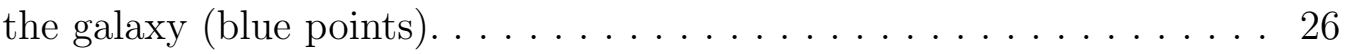

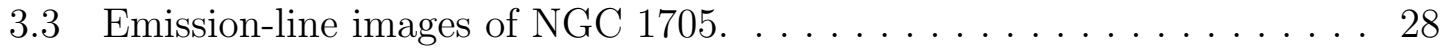

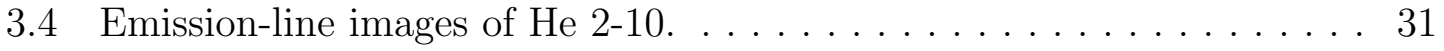

3.5 Emission-line images of NGC 1482 . . . . . . . . . . . . . . . 33

3.6 Shock-corrected ionization parameter map of $[\mathrm{SIII}] /[\mathrm{SII}] \ldots \ldots$. . . . . . 35

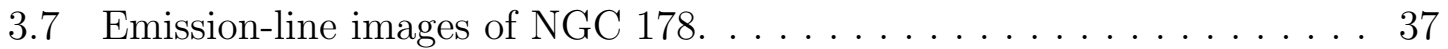

3.8 Emission-line images of NGC $7126 \ldots \ldots \ldots \ldots \ldots \ldots$

3.9 Emission-line ratio maps of $[\mathrm{SIII}] /[\mathrm{SII}]$ for the galaxies in our sample. . 47

3.10 Emission-line ratio maps of $[\mathrm{SII}] / \mathrm{H} \alpha$ for the galaxies in our sample. . . 48

3.11 Emission-line ratio maps of $[\mathrm{SIII}] / \mathrm{H} \alpha$ for the galaxies in our sample. . . 49

4.1 MCELS images for the objects in our sample. . . . . . . . . . . 58

4.2 Stellar spectra from the ionizing stars of the HiI regions. . . . . . . . . 59

4.3 Nebular spectra of the Hir regions in our sample for 3600-4500 А. . . . 60

4.4 Ratio between the predicted and observed emission-line intensities for MCELS L $28 . \ldots \ldots \ldots \ldots \ldots \ldots \ldots \ldots$ 
4.5 Predicted line strengths of [OIII] vs. [OII] and [OII] vs. HeI for

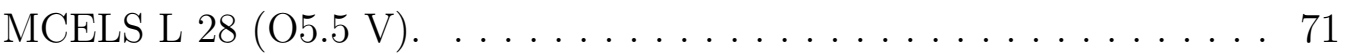

4.6 Same as Figure 4.5 but for MCELS L 43 (O8 V). . . . . . . . . . . . . 72

4.7 Predicted emission-line ratios of $[\mathrm{OIII}] \lambda 5007 / \mathrm{H} \beta$ and $[\mathrm{OII}] \lambda 3727 / \mathrm{H} \beta$ as a function of metallicity are shown for simulations of MCELS L 28 ionized by WM-basic atmospheres with $T_{\text {eff }}=39-43 \mathrm{kK}$ (thin lines). . . 73

4.8 The ratio of the predicted to observed emission-line intensities is plotted as a function of the ionization potential of the emission line for the simulation that has the closest match to our observations. . . . . . . . 93

4.9 Same as Figure 4.8, but for MCELS L 344, MCELS L 43, MCELS L 390, and MCELS L $394 \ldots \ldots \ldots \ldots \ldots$. . . . . . . . . . . . . . . 94

4.10 Same as Figure 4.8, but for MCELS L 032, MCELS L 035, MCELS L 345, and MCELS L $346 . \ldots \ldots \ldots \ldots \ldots \ldots$

$4.11[\mathrm{OI}] \lambda 6300 / \mathrm{H} \beta$ and $[\mathrm{SiI}] \lambda 6716 / \mathrm{H} \beta$ vs. HeI $\lambda 6876 / \mathrm{H} \beta$ for MCELS L 28. . 96

$4.12 F_{\text {pre }} / F_{\text {obs }}$ as a function of spectral type for [OII] $\lambda 3727,[\mathrm{NII}] \lambda 6584$, [OIII] $\lambda 5007$ and [NeIII] $\lambda 3869$ in panels $a-d$, respectively. . . . . . . 97

4.13 SEDs for stars with $T_{\text {eff }}=41,000 \mathrm{~K}$ and $\log \left(L_{\mathrm{bol}}\right)=37.09$ (top) and $T_{\text {eff }}=35,000 \mathrm{~K}$ and $\log \left(L_{\mathrm{bol}}\right)=37.78$ (bottom) for each stellar atmosphere model. . . . . . . . . . . . . . . . . . . . . . . 98

4.14 A comparison of the $T_{\text {eff }}$ from this paper to different $S p T-T_{\text {eff }}$ calibrations. 99 


\section{LIST OF TABLES}

$\underline{\text { Table }}$

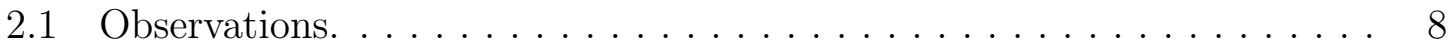

3.1 Sample of Local Starbursts . . . . . . . . . . . . . . . . . . . . . 22

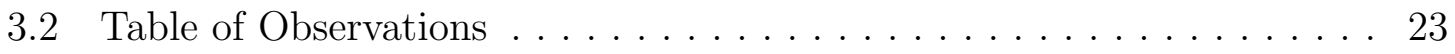

3.3 Star Formation Rates . . . . . . . . . . . . . . . . . . . . . . 41

4.1 Observed Nebular and Stellar Properties _. . . . . . . . . . . . . . 55

4.2 De-reddened Emission Lines Fluxes and Derived Properties . . . . . . . 61

4.3 Parameters of the Best Fit Simulations. . . . . . . . . . . . . . . . 74

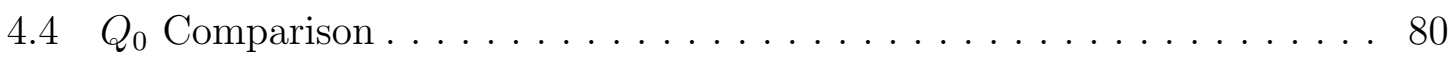




\section{ABSTRACT}

The ionizing light emitted by massive stars represents a critical feedback process in the universe, from potentially providing the ionizing photons for cosmic reionization to forming the basis for diagnostics of fundamental galactic properties. However, the ultimate fate of ionizing photons in a galaxy and the energy distribution of this radiation remain uncertain. The radiation is challenging to study directly; instead, we use both narrowband images and long-slit spectra of photoionized gas to investigate the galactic properties that control the fate of ionizing radiation and to evaluate the spectral energy distributions (SED) predicted by stellar atmosphere codes. First, we generate $[\mathrm{S} \mathrm{III}] /[\mathrm{S} \mathrm{II}]$ maps to trace the passage of ionizing radiation through seven starburst galaxies. With these maps, we discover optically thin ionization cones in NGC 3125 and NGC 5253. Both ionization cones are narrow, which indicates that the low-density passageways carved by wind and bubble activity are narrow as well. These results suggest that an orientation bias limits the ability to directly detect escaping Lyman continuum in starburst galaxies. Next, we turn our attention to the shape of the ionizing SED; we run photoionization simulations drawing from four different grids of atmosphere models for the ionizing source. We compare the emission-line spectra predicted by these model HII regions to long-slit observations of single-star HII regions. All families of atmosphere codes reproduce the observed emission-lines for lines with ionization potential $<35 \mathrm{eV}$, assuming the gas distribution is clumpy. However, the predictions exhibit significant scatter about the observations for ions with higher ionization potential. Overall, we find that atmospheres generated with the WM-basic code best represent the ionizing stars. Comparing the H-alpha derived ionizing photon rates to those from the simulations reveals an offset between the different atmosphere models that is systematic with the hardness of the SEDs. 
Finally, we show that comparing the effective temperatures derived from nebular modeling to those expected from literature calibrations is a potential diagnostic test for the upper layers of the atmosphere models. With this thesis, we demonstrate the diagnostic power of two under-utilized methods: ionization parameter mapping and photoionization simulations of single-star HII regions. 


\section{CHAPTER 1}

\section{Introduction}

The radiation emitted by massive stars represents a critical feedback process in the universe. These stars emit most of their light at energies high enough to ionize interstellar gas, thereby generating bright nebulae known as HII regions. Since the distinctive emission-line spectra of these nebulae are sensitive to the both ionizing stellar continuum and the gas density and abundance, they provide many diagnostics for important physical properties. Among these properties are star formation rates (e.g., Kennicutt et al., 2000), stellar populations and the IMF (e.g, Baldwin et al., 1981; Kaler, 1978; Rigby \& Rieke, 2004; Stasińska \& Leitherer, 1996; Copetti et al., 1986; Dufour, 1975), and gas phase abundances, which inform chemical evolution models (e.g., Kewley \& Dopita, 2002; Edmunds \& Pagel, 1984; Bresolin et al., 1999). As radiation moves away from the star, the ionizing photons shape the interstellar medium (ISM) through both ionization of dense gas and radiation pressure from the photon flux. These processes help carve out low-density bubbles in the ISM and, in conjunction with stellar winds and supernovae, can launch galactic winds, which inject both both energy and material from a galaxy into the intergalactic medium (IGM) (e.g., Murray et al., 2011; Veilleux et al., 2005; Heckman et al., 1990; Mac Low \& McCray, 1988). If enough ionizing photons escape the galaxies themselves, radiation from massive stars may be responsible for ionizing the IGM in the early universe - a process known as cosmic reionization.

The fate of ionizing photons is an important issue, both for our understanding of galaxies role in cosmic reionization and for HII region diagnostics. The classical assumption is that all of the ionizing radiation emitted by massive stars is absorbed by the surrounding HII region, in which case the HII region is optically thick or 
radiation bounded. However, using the Magellanic clouds, Pellegrini et al. (2012) showed that collectively up to $\sim 50 \%$ of the ionizing radiation ultimately escapes the host HII regions of a galaxy. These photons go on to provide a significant portion of the ionization budget for the diffuse, warm ionized medium of a galaxy. The fraction of ionizing radiation that actually escapes a galaxy itself is a major linch pin in the debate over high-redshift star-forming galaxies as the source for cosmic reionization.

In our current paradigm of massive-star feedback, massive stars carve out low density bubbles in the ISM. If the bubble has enough energy, it can break out of the galaxy, forming a galactic fountain or, in some cases, a galactic wind (e.g., Mac Low \& McCray, 1988; Dove et al., 2000; Fujita et al., 2003). In galaxies for which the star formation rate is high enough, this feedback could essentially shred the ISM, making the galaxy porous to ionizing radiation (Clarke \& Oey, 2002). However, observational evidence for this explanation of the escape of Lyman continuum from starbursts remains inconclusive.

In order to provide enough ionizing radiation to reionize the universe by redshift six, the average escape fraction from all galaxies needs to be greater than $20 \%$ (e.g., Bouwens et al., 2012). At intermediate redshift ( $\mathrm{z} \sim 3)$, there is observational evidence that at least some starbursts are leaking significant fractions of ionizing radiation (e.g., Steidel et al., 2001). However, studies involving large samples of these highredshift starbursts find that only $\sim 10 \%$ of such galaxies have direct evidence for escaping Lyman continuum (e.g., Shapley et al., 2006; Iwata et al., 2009). Estimates for the average escape fraction from LBGs at $z=3$ are as low as $5 \%$ and go up to $20 \%$ (Vanzella et al., 2010). At lower redshifts there is indirect evidence that some starbursts have low optical depth to the Lyman continuum (Heckman et al., 2011). Yet, only two galaxies at redshift zero have direct detections of escaping Lyman continuum (Leitet, 2013, 2011), and composite studies of $z \sim 1$ galaxies do not detect escaping Lyman continuum (e.g., Siana et al., 2007, 2010; Cowie et al., 2009). Until the galactic properties and processes that regulate escape of ionizing radiation are well understood, the role of galaxies in cosmic reionization will remain an open question. 
Beyond the ultimate fate of ionizing radiation, the spectral energy distribution (SED) of the ionizing radiation is a crucial component for interpreting observed emission-line ratios. The emission-line spectrum of photoionized gas depends critically on the shape of the ionizing SED. In order to describe the massive-star SED, we depend on codes that model the radiative transfer through the stellar atmosphere. These codes incorporate complicated stellar physics, which include conditions that are not in local thermodynamic equilibrium, the effects of metal lines on the transmitted spectrum, and the effects of stellar winds. Research groups choose different algorithms to balance careful treatment of the physics with reasonable computing times. As a consequence, the derived HII region diagnostics will depend on which stellar atmosphere code is chosen. For example, Voges et al. (2008) showed that the rate of ionizing photons predicted by different atmosphere codes can vary by up to 0.5 dex, which directly affects star formation rates derived using the $\mathrm{H} \alpha$ emission line. Another example is the spectral type to stellar effective temperature calibration, which allows one to translate between spectral type, an observational classification, to effective temperature, an intrinsic stellar property. The commonly used Vacca et al. (1996) calibration, which is based on unblanketed models, predicts $T_{\text {eff }}$ that are a few thousand degrees hotter than calibrations that use more recent line-blanketed models (e.g., Martins et al., 2005; Massey et al., 2005). It is essential to understand which of the currently available models best represent the true ionizing SED.

Directly measuring both the fraction of ionizing radiation that escapes a galaxy and the shape of the ionizing SED is challenging. The radiation itself falls in the extreme ultraviolet, which is a wavelength range that is exceedingly difficult to observe. Not only does it require space-based observations, but intervening gas and dust will heavily absorb the flux. For observations at high redshift, the Lyman continuum shifts to the optical and infrared, which alleviates some of the observational challenges. However, the signal is low and experiences significant absorption by lineof-sight H. Generally, one must either observe the objects for a long time or stack observations of many galaxies in order to get a detection.

Instead of trying to directly observe Lyman continuum, we can take advantage 
of the close relationship between the emission lines emitted by photoionized gas and the ionizing SED. As the hardness and quantity of ionizing photons change, so too will the strength of the observed emission lines. Thus, if one knows the gas density, morphology, and abundance, the other parameters on which the emission lines depend, the observed lines will constrain the shape of the SED (e.g., Oey et al., 2000; Crowther et al., 1999; Esteban et al., 1993). Furthermore, as ionizing radiation moves through the ISM, the line ratios change relative to each other. Thus, the ionization structure, as revealed by ionization parameter maps, will trace the passage of ionizing photons as they move through the galaxy (e.g., Pellegrini et al., 2012). This approach has the distinct advantage of being observationally straightforward.

In this thesis, we use both narrowband images and long-slit spectra of photoionized gas to investigate the galactic properties that control the fate of ionizing radiation and to evaluate the SEDs predicted by currently available stellar atmosphere codes. First, we present a case study of one nearby starburst, for which we discovered an ionization cone extending out of the galaxy away from the central star-forming region. This discovery illustrates the potential of using emission-line ratio maps to trace the passage of ionizing radiation in a galaxy (e.g., Pellegrini et al., 2012; Zastrow et al., 2011). We then examine emission-line ratio maps of six other nearby starbursts and use the ionization structure to evaluate the optical depth to Lyman continuum. With these maps, in conjunction with ancillary data, we explore the observational biases that challenge observations of escaping Lyman continuum and whether these galaxies fit our current paradigm for how ionizing radiation may escape a galaxy. Finally, we use long-slit observations of a sample of single-star HII regions to probe the shape of the massive star SED. Using stellar atmosphere models as the ionizing source, we run photoionization simulations to generate model HII regions. We then compare the predicted emission-line spectra to the observed spectra and evaluate which stellar atmosphere models best represent the true ionizing stars. The results of this work, demonstrate the diagnostic power of two under-utilized methods: ionization parameter mapping and photoionization simulations of single-star HII regions. 


\section{CHAPTER 2}

\section{An Ionization Cone in NGC 5253}

\section{$2.1 \quad$ Introduction}

The fate of ionizing photons in starburst galaxies is a critical issue for our understanding of cosmic reionization. Early-epoch star-forming galaxies are the most likely candidate source for the required ionizing radiation (e.g., Madau et al., 1999; Fan et al., 2001). However, quantifying the escape fraction of ionizing radiation $\left(f_{\text {esc }}\right)$ has proven to be challenging.

Generally, the interstellar medium (ISM) is optically thick to Lyman continuum. As discussed by Heckman et al. (2011), the average hydrogen column density in galaxies ranges from a factor $10^{4}$ to $10^{7}$ times higher than the column that produces unity optical depth in the Lyman continuum. Thus, $f_{\text {esc }}$ will be dependent on the morphology of the ISM. For example, models have shown that a clumpy ISM yields a higher $f_{\text {esc }}$ than a smoothly varying medium (e.g., Ciardi et al., 2002; Fernandez \& Shull, 2011). For ionizing radiation to escape, low density paths out of the galaxy must be created. In starburst galaxies, the mechanical feedback from massive stars creates low density bubbles in the ISM, which can then go on to break out of the galactic disk, facilitating the escape of radiation (e.g., Mac Low \& McCray, 1988; Clarke \& Oey,

2002). However, the influence of feedback on $f_{\text {esc }}$ is not straightforward. Simulations show that the very shells and bubbles that generate low-density holes in the ISM can initially trap ionizing photons before break-out, which will affect $f_{\text {esc }}$ (Fujita et al., 2003; Dove et al., 2000).

Understanding this problem is complicated by the small number of galaxies detected with significant $f_{\text {esc }}$, despite large effort by the community to measure it both 
locally and at high redshift. Direct detection of excess Lyman continuum has been found in a subset of $z \sim 3$ Lyman-break galaxies (e.g. Steidel et al., 2001). However, the fraction of galaxies with high $f_{\text {esc }}$ is low, on the order of $10 \%$ (e.g., Iwata et al., 2009; Shapley et al., 2006). For local starbursts, the detection rate drops, and most studies obtain upper limits to $f_{\text {esc }}$ of a few percent (e.g., Leitherer et al., 1995; Heckman et al., 2001; Grimes et al., 2009; Siana et al., 2010).

One possible explanation for the low detection rate is an orientation bias. In the paradigm outlined above, feedback processes will create passageways perpendicular to the galaxy plane. It follows that ionizing photons will preferentially escape in that direction with an opening angle dependent on galactic conditions (Dove et al., 2000; Gnedin et al., 2008; Fernandez \& Shull, 2011). Direct measurements of $f_{\text {esc }}$ will thus depend on the orientation of the galaxy to our line-of-sight. This model is conducive to the formation of photoionized ionization cones. While photoionized emission has been observed in outflows near starburst nuclei (e.g., Sharp \& Bland-Hawthorn, 2010; Shopbell \& Bland-Hawthorn, 1998), clear evidence for ionization cones extending well into the galaxy halo has not yet been observed. Here, we report a new detection of such an ionization cone in a starburst galaxy, NGC 5253. Its narrow opening angle suggests that orientation may play an important role in the detectability of escaping Lyman continuum radiation.

\subsection{Observations}

NGC 5253 is a nearby, dwarf galaxy undergoing intense, centrally concentrated star formation. The most recent episode of star formation produced massive super star clusters with ages of just a few Myr (e.g., Calzetti et al., 1997). At a distance of 3.8 Mpc (Sakai et al., 2004), we are able resolve structure down to tens of parsecs. NGC 5253 is well studied across many wavelengths, providing much ancillary data to augment this study.

To identify channels where Lyman continuum radiation might escape from the galaxy, we apply the technique of ionization parameter mapping (e.g., Pellegrini et al., in preparation; Pogge, 1988). The ionization parameter is a measure of the ionizing 
radiation energy density relative to the gas density, and it can be mapped by emissionline ratios that probe high- vs low-ionization species. Regions that are optically thick to ionizing radiation show a low-ionization boundary between the highly ionized regions close to the ionizing source and the neutral environment; whereas optically thin regions maintain high excitation throughout. For this study, we use [S III] $\lambda 9069$ and [S II] $\lambda 6716$ to map the high- and low-ionization species, respectively.

We obtained narrow-band images of NGC 5253 on the nights of 2009 July 9-11 using the Maryland-Magellan Tunable Filter (MMTF; Veilleux et al., 2010). The bandpasses include $\mathrm{H} \alpha$ and narrow-band continuum imaging observed at the redshift of NGC 5253. They are given in Table 2.1, together with their exposure times. MMTF uses a low-order Fabry-Perot etalon and is mounted on the imaging spectrograph, IMACS, of the Magellan Baade telescope at Las Campanas Observatory. The IMACS $\mathrm{f} / 2$ camera has an $8 \mathrm{~K} \times 8 \mathrm{~K}$ Mosaic $\mathrm{CCD}$ with a pixel scale of $0.2^{\prime \prime}$ per pixel. For these observations, MMTF was used at low etalon spacings to provide a monochromatic field of view of $11.5^{\prime}$. We additionally obtained a long-slit spectrum of NGC 5253 . The seeing conditions ranged from $0.5^{\prime \prime}-0.7^{\prime \prime}$ for the $[\mathrm{S} \mathrm{II}]$ and $[\mathrm{S} \mathrm{III]} \mathrm{images} \mathrm{and}$ $1.5^{\prime \prime}-2^{\prime \prime}$ for $\mathrm{H} \alpha$.

The bias subtraction, flat-fielding, and sky subtraction are accomplished using version 1.4 of the MMTF data reduction pipeline, ${ }^{1}$ described in Veilleux et al. (2010). The $\mathrm{H} \alpha$-continuum image is first flux calibrated using photometry from standard star observations. Then, the long slit spectrum is corrected for instrument response using standard star spectra, and flux calibrated using the flux-calibrated, $\mathrm{H} \alpha$-continuum image. Finally, the other emission-line images are normalized to the emission measured across each bandpass in the flux-calibrated, long slit spectrum. Systematic errors for the flux calibration are about a factor of two. The images are registered and rescaled using standard IRAF ${ }^{2}$ tasks, then continuum subtracted. Galactic extinction is corrected assuming $E(B-V)=0.0475$ across the field (Burstein \& Heiles, 1982) and

\footnotetext{
${ }^{1}$ http://www.astro.umd.edu/ ${ }^{\sim}$ veilleux/mmtf/datared.html

${ }^{2} \mathrm{IRAF}$ is distributed by NOAO, which is operated by AURA, Inc., under cooperative agreement with the National Science Foundation.
} 
Table 2.1. Observations

\begin{tabular}{|ccccc|}
\hline $\begin{array}{c}\text { Emission } \\
\text { line }\end{array}$ & $\begin{array}{c}\text { Wavelength } \\
(\AA)\end{array}$ & $\begin{array}{c}\text { Effective } \\
\text { Bandpass }(\AA)\end{array}$ & Exp. Time & $\begin{array}{c}\text { Flux }^{a} \\
\left(10^{-13} \mathrm{erg} \mathrm{s}^{-1} \mathrm{~cm}^{-2}\right)\end{array}$ \\
\hline$[$ SiII $]$ & 9081 & 27.0 & $5 \times 1200 \mathrm{~s}$ & 0.39 \\
continuum & 9180 & 27.0 & $5 \times 1200 \mathrm{~s}$ & $\ldots$ \\
{$[$ Sii $]$} & 6726 & 16.2 & $5 \times 1200 \mathrm{~s}$ & 0.59 \\
continuum & 6680 & 16.2 & $5 \times 1200 \mathrm{~s}$ & $\ldots$ \\
H $\alpha$ & 6572 & 18.5 & $3 \times 1200 \mathrm{~s}$ & 12.9 \\
continuum & 6680 & 18.5 & $3 \times 1200 \mathrm{~s}$ & $\ldots$ \\
\hline
\end{tabular}

Note: ${ }^{a}$ Continuum-subtracted flux measured in the ionization cone using the

DS9 funtools module.

the extinction law from Cardelli et al. (1989). The internal extinction is known to be variable throughout the galaxy (Calzetti et al., 1997; Caldwell \& Phillips, 1989; Cresci et al., 2005), and its effects are discussed in $\S 3.1$.

A three-color composite of our data is presented in Figure 2.1, along with the continuum-subtracted, emission-line images. Sky background and noise properties of the images are measured using the median and standard deviation in the flux of fifteen $20^{\prime \prime} \times 20^{\prime \prime}$ boxes around the galaxy. In $\mathrm{H} \alpha$, we have a $3 \sigma$ confidence detection limit of $4.1 \times 10^{-18} \mathrm{erg} \mathrm{s}^{-1} \mathrm{~cm}^{-2}$. The ionized gas in the galaxy has a roughly spherical distribution around the nucleus, with networks of loops and filaments particularly noticeable to the south and northwest (e.g., Marlowe et al., 1995).

\subsection{Ionization Cone in NGC 5253}

\subsubsection{Morphology and Excitation of the Ionization Cone}

NGC 5253 shows a stunning detection of an ionization cone in a starburst galaxy. In Figure 2.1, the ionization cone stands out beautifully, extending ESE along the minor axis. In the ratio map, Figure 2.2, the ionization cone is identified by its high excitation relative to the rest of the galaxy. This high excitation has also been observed in $[\mathrm{OIII}] /[\mathrm{SII}]$ (Calzetti et al., 1999). It has an opening angle $<40^{\circ}$ with a narrow appearance at large radii. As the distance from the galaxy increases, the morphology of the cone becomes filamentary. While the cone appears to be onesided, we note that extended [SIII] emission to the northwest may suggest a bi-cone 


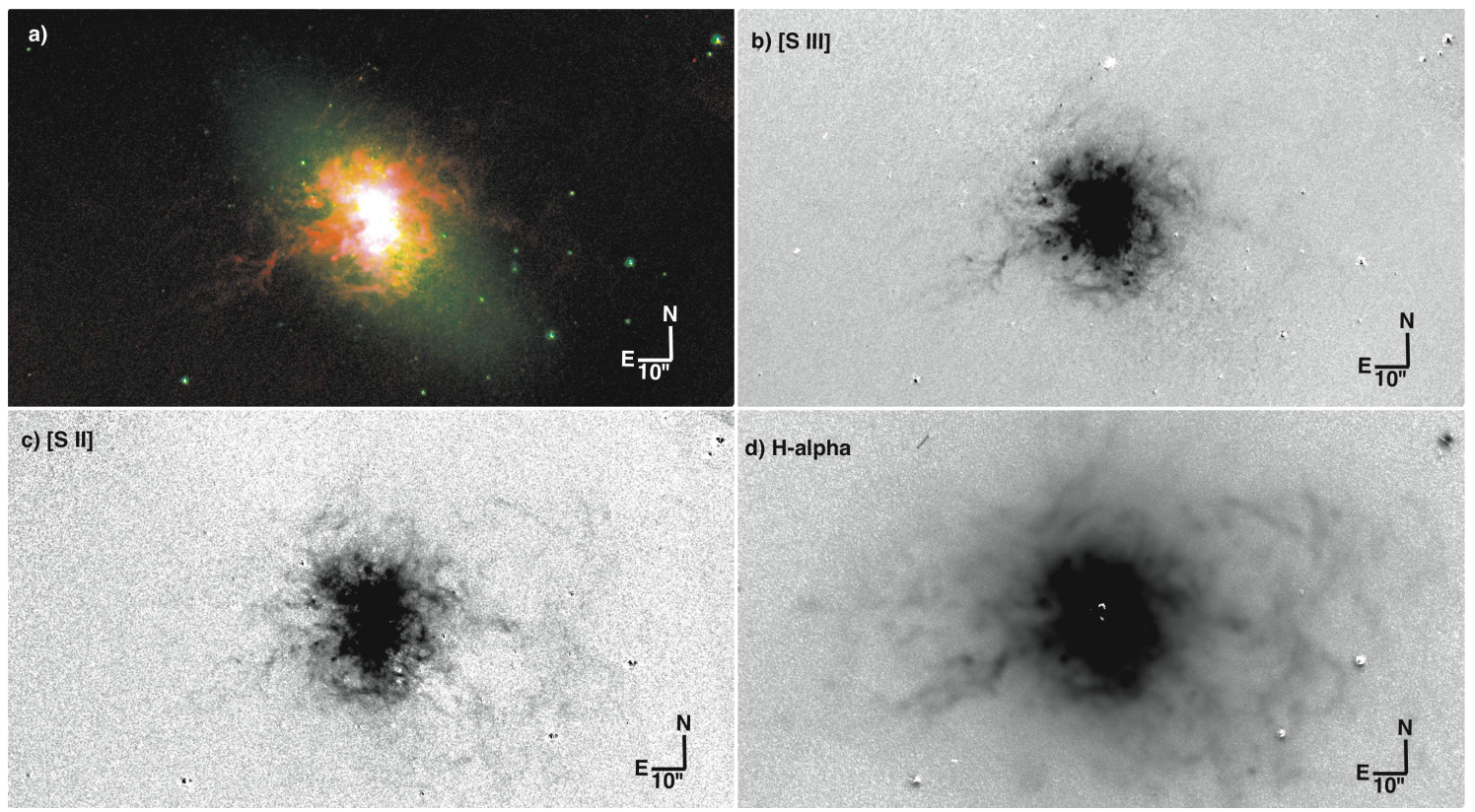

Figure 2.1. a) Composite image of NGC 5253. Red, blue and green correspond to [SIII], [SII], and continuum at $\lambda 6680$, respectively. Ionization cone extends ESE from the nuclear starburst. Panels $b$, $c$, and $d$ show individual continuum-subtracted, [SIII], [SII], and $\mathrm{H} \alpha$, respectively. At a distance of $3.8 \mathrm{Mpc}, 10^{\prime \prime}=180 \mathrm{pc}$.

(Figure 2.1).

The changes in ionization parameter as a function of radius are shown more quantitatively in Figure 2.3. These plots are generated using $3 \times 3$ binning of the continuum-subtracted images, and a $3 \sigma$ detection cut-off in all three emission lines. The ionization cone, shown in red, is defined as the region between P.A. $=102^{\circ}-140^{\circ}$ using $\alpha=13^{\mathrm{h}} 39^{\mathrm{m}} 55^{\mathrm{s}} .7, \delta=-31^{\circ} 38^{\prime} 24^{\prime \prime}(\mathrm{J} 2000)$ as the apex. This point, marked by the cross in Figure 2.2, is the location of a likely source for the ionizing radiation, as discussed below. For comparison, the blue line is representative of the rest of the galaxy.

The top panel of Figure 2.3 clearly shows a strong excess in the [SIII]/ [SII] ratio for the ionization cone at lower galactocentric radii $R$, and a clear excess that continues to $R \sim 600-700 \mathrm{pc}$. The middle and lower panels show that this excess cannot be explained solely by the well-known increasing gradient in $[\mathrm{SII}] / \mathrm{H} \alpha$ that is found in the diffuse, ionized gas of star-forming galaxies (e.g., Rand, 1998; Haffner et al., 1999). Furthermore, Figure 2.1 shows that the primary star-forming region extends 


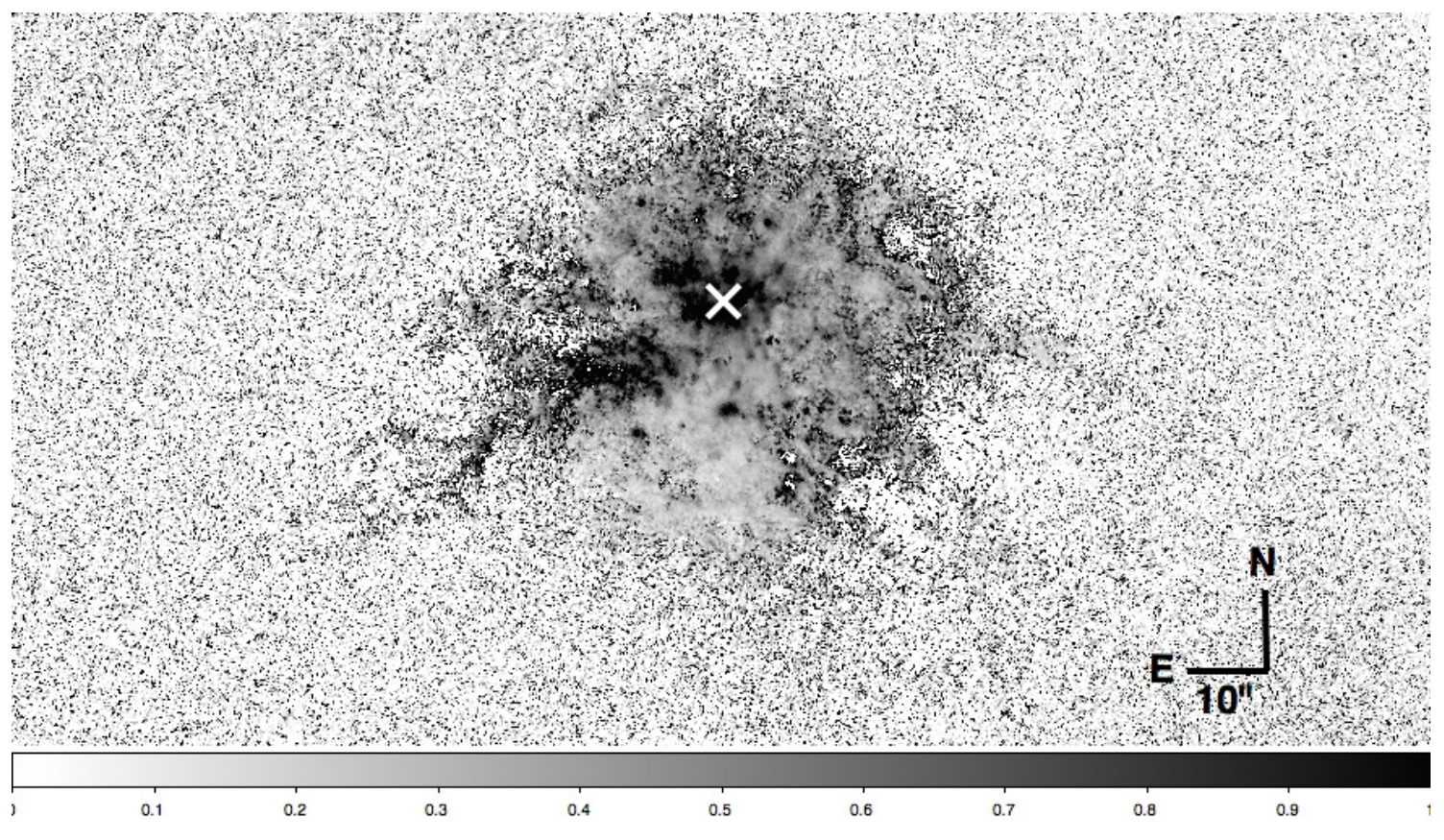

Figure 2.2. [SIII]/ [SII] ratio map for NGC 5253. Dark indicates high values of [SIII]/ [SII].

only to $\sim 500 \mathrm{pc}$, whereas photoionized material associated with the cone clearly extends beyond. This strongly suggests that there is not enough gas in the outer halo to absorb all the ionizing photons, and that they therefore may escape the galaxy. We note in Section 2.3.2 that NGC 5253 is not rotationally supported, which suggests that this starburst is undergoing, or has recently experienced, a merger or interaction with another galaxy (e.g., López-Sánchez et al., 2012; Kobulnicky \& Skillman, 2008). In the case of merger activity there might be additional extraplanar gas that has not been cleared by feedback activity. This additional gas would make it more challenging for ionizing radiation to escape the starburst. However, the ionization cone in NGC 5253 does provide an example of the manner in which ionizing radiation may escape starbursts, regardless of the ultimate destination of that radiation.

As noted above, the extinction throughout the starburst is variable (Cresci et al., 2005), and might enhance the observed [SIII]/ [SII] ratio relative to the intrinsic one. In particular, a dust lane with associated CO along the minor axis is coincident with the location of the cone (Calzetti et al., 1997; Caldwell \& Phillips, 1989; Meier et al., 2002). However, Figure 3 shows that we need $A_{V} \sim 3-10$ mag to explain the 


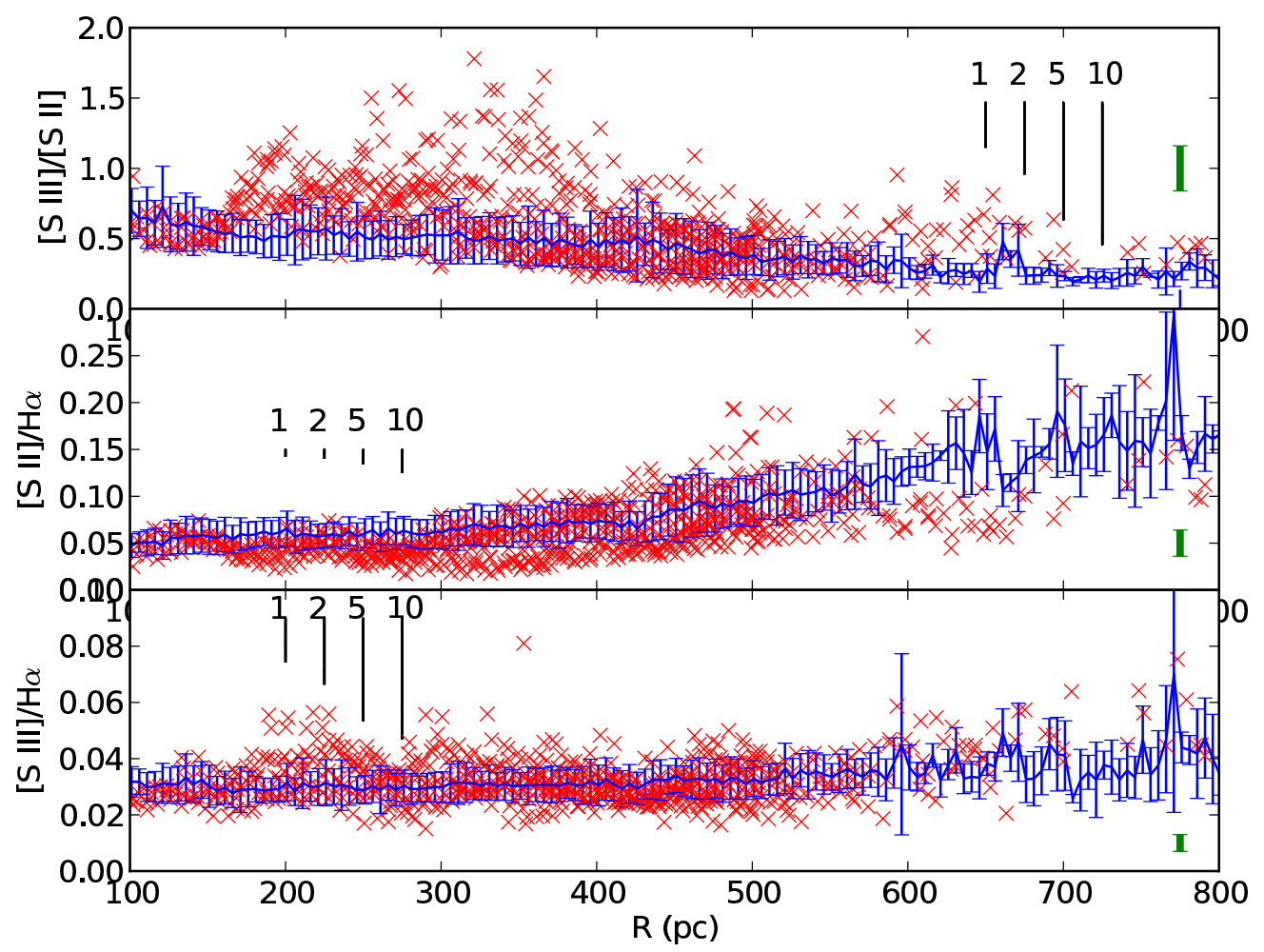

Figure 2.3. Emission-line ratios vs. galactocentric radius. Red represents ionization cone emission. Blue line is the mean galactic value in radial bins, with bars noting one standard deviation. Representative measurement error is shown by the green error bar. The black lines indicate the effect of different levels of extinction $\left(A_{V}=1,2,5,10\right)$. Top, middle, and bottom panels show [SIII]/ [SII], $[\mathrm{SII}] / \mathrm{H} \alpha$, and $[\mathrm{SIII}] / \mathrm{H} \alpha$, respectively.

observed $[\mathrm{SIII}] /[\mathrm{SII}]$ ratios entirely by the dust lane, which is much more than its observed extinction $\left(A_{V} \sim 2.2 \mathrm{mag}\right.$, Calzetti et al., 1997). If there were that much extinction, we would expect a much lower flux along the cone, which is not seen in the emission-line images in Figure 2.1. Additionally, the $[\mathrm{SII}] / \mathrm{H} \alpha$ ratio observed in the cone cannot be explained by extinction. We also note that the highest extinction will likely be strongest at the smallest $R$.

Another possible effect is a bias in our observed [SIII]/ [SII] ratio caused by strong variations in electron density. This affects the [SII] $\lambda 6716 / \lambda 6731$ ratio, so that our observed $\lambda 6716$ may underestimate the total [SII] at high density. This error again will be largest at smaller radii where densities are larger, and leads to difference of $\sim 25 \%$ between a density of $10^{2}$ and $10^{3} \mathrm{~cm}^{-3}$. In our long-slit spectrum, [SII] 
$\lambda \lambda 6716 / 6731$ ratio ranges from 1.4 to 1.0, which correspond to densities of $\lesssim 100 \mathrm{~cm}^{-3}$ and $\sim 500 \mathrm{~cm}^{-3}$, respectively. Since this is a smaller variation than between $10^{2}$ and $10^{3} \mathrm{~cm}^{-3}$, the error on our observed [SII] due to density variations is likely much less than $25 \%$.

The measured emission-line fluxes in the ionization cone (Table 2.1) can set limits on the ionizing source. The $\mathrm{H} \alpha$ flux corresponds to an $\mathrm{H} \alpha$ luminosity $L(\mathrm{H} \alpha)=$ $2.2( \pm 1.1) \times 10^{39} \mathrm{erg} \mathrm{s}^{-1}$. This is $\sim 6 \%$ the total $L(\mathrm{H} \alpha)$ in the galaxy (Marlowe et al., 1995 , corrected for distance), from which we obtain a lower limit of $\log \left(Q_{0}\right) \geq 51.19$ on the rate of ionizing photons, $Q_{0}\left(\mathrm{~s}^{-1}\right)$, needed to explain the observed emission. An O7 star with the $\sim 0.2 Z_{\odot}$ metallicity of NGC 5253 (Walsh \& Roy, 1989) has $\log \left(Q_{0}\right)=49.0$ (Smith et al., 2002). This implies that radiation equivalent to that produced by $\gtrsim 160 \pm 80 \mathrm{O} 7$ stars is needed to ionize the gas. This is a lower limit for the radiation escaping the nuclear starburst, since the flux will be affected by internal extinction and $f_{\text {esc }}$.

NGC 5253 is host to many young clusters that could be the source for the ionizing radiation illuminating the cone. Two likely candidates are NGC 5253-5 (Calzetti et al., 1997) and the ionizing cluster of the radio supernebula (Beck et al., 1996; Turner et al., 2000; Gorjian et al., 2001). The location of these objects, separated by just a few arcseconds on the sky (Alonso-Herrero et al., 2004), is noted by the cross in Figure 2.2. Both clusters are very young and very massive (Turner et al., 2000; Calzetti et al., 1997; Alonso-Herrero et al., 2004). In fact, the radio supernebula may be gravitationally confined (Turner et al., 2003). The $20 \mathrm{pc}$ region around the radio supernebula hosts an ionizing population $\sim 7000$ O7 stars, with some uncertainty due to the high extinction towards this cluster (Turner \& Beck, 2004). Thus, the clusters in NGC 5253 easily generate enough ionizing flux to explain the ionization cone.

\subsubsection{Kinematics}

The HI kinematics in NGC 5253 show only small scale rotation about the minor axis (Kobulnicky \& Skillman, 2008), making it unlikely that NGC 5253 is rotationally supported (Caldwell \& Phillips, 1989). In Figure 2.4, the line profiles from an $\mathrm{H} \alpha$ 


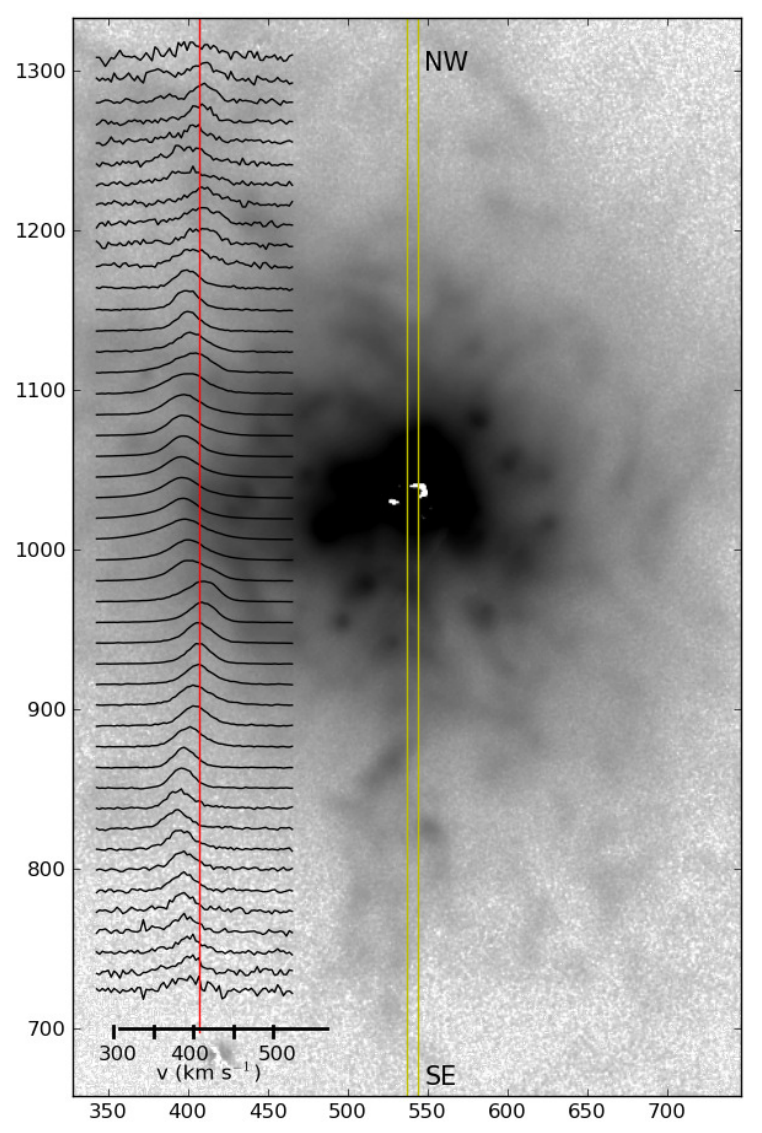

Figure 2.4. NGC $5253 \mathrm{H} \alpha$ image, minor axis oriented along the $y$-axis. $\mathrm{H} \alpha$ echelle profiles are overlaid to the left. The profiles are normalized to a peak of one and the spacing between each profile is $2 . " 6$. The red line denotes the $v_{\text {sys }}$ for NGC 5253 , reported to be $407 \pm 3 \mathrm{~km} \mathrm{~s}^{-1}$ (Koribalski et al., 2004). The slit position is marked in yellow.

echellegram (Slit 3, Martin \& Kennicutt, 1995) are overlaid on the $\mathrm{H} \alpha$ image of NGC 5253. The velocity shows significant variation with position, and a maximum projected velocity difference $\Delta v \sim 30 \mathrm{~km} \mathrm{~s}^{-1}$ relative to systemic.

An expanding wind might explain the kinematics. In this scenario, we expect the velocity to increase with distance from the galaxy, since the wind is expanding into the lower density gas of the halo. To the southeast, we do see an increase in velocity with galactocentric distance. However, it reaches a maximum at $\sim 40^{\prime \prime}$ from the galaxy center before decreasing towards systemic at larger $R$. This does not 
strictly follow what is expected for a free-flowing wind, in which the velocity should continue to increase. Additionally, an expanding wind typically exhibits line splitting, which is not seen in the $\mathrm{H} \alpha$ profiles (Martin \& Kennicutt, 1995). Kinematic data from HI observations are suggestive of either inflow or outflow along the minor axis (Kobulnicky \& Skillman, 2008). Therefore, although not completely straightforward, mechanical feedback might plausibly explain the observed kinematics based on the limited information available.

\subsubsection{Possible AGN}

Typically, ionization cones are associated with AGN, which suggests an alternative explanation for the ionization cone in NGC 5253. The morphology of the cone is very narrow. This suggests collimation of the ionizing radiation, as would be expected if there is an AGN present. The radio supernebula is particularly intriguing in this context. Only $\sim 0 . " 6$ in size, it provides roughly $80 \%$ of the galaxy's IR flux at $12 \mu \mathrm{m}$ (Gorjian et al., 2001). There is some debate about whether a young super star cluster or AGN is the ionizing source of this nebula. Its radio continuum has a flat slope, consistent with thermal emission (Beck et al., 1996; Turner et al., 1998, 2000), but which does not rule out an AGN component (Turner et al., 1998). However, the observed spectral lines are also narrower than would be expected from an AGN (Beck et al., 1996). Additionally, X-ray observations of NGC 5253 show that the point sources and diffuse emission are consistent with star formation and shocks (Ott et al., 2005; Summers et al., 2004; Martin \& Kennicutt, 1995). Therefore, while we cannot rule out an AGN in NGC 5253, there are challenges facing that interpretation.

If NGC 5253 has an obscured AGN, a precessing jet might explain the gas kinematics. In this case, the velocities on one side of the galaxy should be mirrored exactly on the opposite side. In NGC 5253, the overall sinusoid in velocity to the southeast is roughly reflected to the northwest. Along the ionization cone, the velocity transitions from redshift to blueshift around $\sim 30^{\prime \prime}$ from the galaxy. To the northwest, the opposite transition, of roughly equal amplitude, is seen at $\sim 28^{\prime \prime}$. However, the reflection is not perfect; there are points to the northwest where the velocity briefly flips, which 
are not seen to the southeast. Additionally, the amount of blueshift to the northwest does not match the redshift observed in the southeast. Therefore, while the general kinematics could be explained by a precessing jet, the discrepancies are difficult to reconcile in detail.

\subsection{Implications: Orientation Bias}

Based on our detection of an ionization cone in NGC 5253, we suggest that an orientation bias can explain the difficulty in detecting escaping ionizing radiation in starburst galaxies. We observe evidence that ionizing radiation from NGC 5253 is traveling to large radii, if not escaping the galaxy entirely. Assuming the cone is axisymmetric, the estimated solid angle subtended by the cone is $\sim 3 \%$ of $4 \pi$ steradians, suggesting that the escape process happens over a small solid angle. If we assume isotropic radiation from 7000 O7 stars, as above, we could expect to see ionized-gas emission in this solid angle equivalent to that produced by $210 \mathrm{O} 7$ stars. This is similar to our estimated $160 \pm 80 \mathrm{O} 7$ stars needed to generate the observed $\mathrm{H} \alpha$ emission in the cone, supporting the physical link between the source and the cone. The narrow morphology suggests that in order to detect Lyman continuum, the line-of-sight to the galaxy must be close to the axis of escape. Thus, the orientation of the galaxy should strongly influence the detectability of Lyman continuum radiation.

We can find further evidence for this scenario in recent observations by Heckman et al. (2011). In their study of 11 Lyman-break analog galaxies and 15 local starbursts, including NGC 5253, they found indirect evidence for significant $f_{\text {esc }}$ in three of the Lyman-break analogs. Outflow velocities on the order of $10^{3} \mathrm{~km} \mathrm{~s}^{-1}$ were found in all the galaxies suggested to have significant $f_{\text {esc }}$. These outflow velocities are so high, that we can interpret it to mean the direction of the outflow must be closely aligned to the lines-of-sight towards the galaxies. Since winds in starburst galaxies are generally coincident with the poles (Veilleux et al., 2005), this suggests that these inferred high $f_{\text {esc }}$ are all associated with face-on systems. This fits our explanation that measurements of large $f_{\text {esc }}$ are biased towards galaxies whose outflow happens along our line-of-sight. 


\subsection{Conclusion}

The ionization cone in NGC 5253 provides a new perspective to our understanding of the fate of ionizing photons in starburst galaxies. This ionization cone appears optically thin, which is suggestive of the escape of ionizing radiation along the cone. At minimum, ionizing radiation is escaping from the nuclear starburst into the galaxy's halo. We considered both stellar and non-stellar sources for the ionization cone. Assuming a stellar source, the massive clusters in NGC 5253 produce enough ionizing radiation to explain the $\mathrm{H} \alpha$ emission observed. However, the possibility of an obscured AGN has not been ruled out. The $\mathrm{H} \alpha$ kinematics along the cone exhibit a complex morphology, but are consistent with some form of outflow. The data are not straightforward to interpret, but might be explained by an expanding wind or perhaps a precessing jet.

Using the ionization cone of NGC 5253 as an analog for other starburst galaxies, we see that ionizing radiation escapes along the minor axis. Additionally, the solid angle over which this radiation escapes is small, based on the cone morphology. This will constrain the viewing angles from which Lyman continuum radiation can be detected. Thus, the orientation of the galaxy will strongly influence the ability to detect Lyman continuum radiation. NGC 5253 may be the nearest starburst galaxy with a significant $f_{\text {esc }}$, and offers a unique opportunity to study the emission mechanisms in detail. 


\section{CHAPTER 3}

\section{New Constraints on the Escape Fraction of Ionizing Photons from Local Starbursts Using [SIII]/[SII] Ionization Parameter Maps}

\subsection{Introduction}

Between $z \sim 11$ and $z \sim 6$, the universe experienced a change of state known as cosmic reionization (e.g., Shull et al., 2012; Fan et al., 2006, 2001). The source for cosmic reionization is still unclear; the primary contenders are star-forming galaxies and quasars. However, the space density of quasars peaks at $z \sim 3$ and drops below the threshold needed to supply the required amount of ionizing radiation at $z \gtrsim 6$ (Madau et al., 1999; Fan et al., 2001; Meiksin, 2005; Shankar \& Mathur, 2007). The massive stars found in high-redshift star-forming galaxies have the potential to provide enough ionizing radiation, but whether they contribute significantly to the intergalactic ionizing emissivity is not yet established (e.g., Yajima et al., 2012). In order to account for cosmic reionization, the mean fraction of ionizing radiation that escapes the galaxy population, $<f_{\text {esc }}>$, needs to be at least $20 \%$ (e.g., Bouwens et al., 2010).

In theory, starburst galaxies should be porous to ionizing radiation (e.g., Clarke \& Oey, 2002; Paardekooper et al., 2011). Stellar winds and supernovae, in conjunction with radiation pressure, will punch holes in the interstellar medium (ISM) (e.g., Mac Low \& McCray, 1988), and thereby create low density passageways through which ionizing radiation may escape (e.g., Dove et al., 2000). Feedback from massive stars is strongest in galaxies with high star-formation rates, making starbursts attractive candidates for the source of cosmic reionization. 
The observational evidence for significant $f_{\text {esc }}$ at high redshift is inconclusive. Using a sample of 29 Lyman break galaxies (LBG), Steidel et al. (2001) showed that at least some $z \sim 3$ starbursts have large escape fractions. However, Lyman continuum (LyC) narrow-band imaging studies with larger galaxy samples found escaping Lyman continuum in only $10 \%$ of the galaxies observed (e.g., Shapley et al., 2006; Iwata et al., 2009). Narrowband LyC observations by Nestor et al. (2011), put the mean $z \sim 3$ escape fraction at $<f_{\text {esc }}>\sim 0.12$. It is important to note that Vanzella et al. (2012) recently illustrated that a majority of narrowband Lyman continuum detections could actually be low redshift interlopers, which would drive the observational $f_{\text {esc }}$ even lower.

At intermediate to low redshift, some evidence exists for significant $f_{\text {esc }}$ from LGB analogs. A subset of these galaxies with extreme feedback have favorable conditions for escaping Lyman continuum, such as low opacity in the ISM (Heckman et al., 2011). However, the fraction of galaxies with these conditions is still small, indicating a low mean $f_{\text {esc }}$ (e.g., Siana et al., 2010). Looking at $z \sim 0$ galaxies, the detection rate drops further, with only two examples of local starbursts that have measurable $f_{\text {esc }}$ (Leitet et al., 2013, 2011). Most other studies find no direct evidence for escaping Lyman continuum in local starbursts (Bridge et al., 2010; Grimes et al., 2009; Heckman et al., 2001; Leitherer et al., 1995).

In order to determine the mean galactic $f_{\text {esc }}$ from observed escape fractions, we need to disentangle the effects of observational biases from trends with intrinsic galaxy properties. In the massive-star feedback-driven model, the escape fraction will depend on the star formation rate, the ISM morphology and density (Fernandez \& Shull, 2011; Benson et al., 2012; Ciardi et al., 2002), and distribution of star formation. However, the winds that clear the ISM are not isotropic, which leads to a preferred direction for escape and finite opening angles (e.g., Veilleux et al., 2005). These effects create observational dependencies with the orientation of the host galaxy. Finally, measuring the Lyman continuum at any redshift is a challenge. Most of the flux is absorbed by the intergalactic medium, thus the signal is low, which requires long integration times. For targets at higher redshift, low-redshift interlopers confuse the statistics 
(Vanzella et al., 2012). Some studies measure the optical depth to UV lines in the ISM in lieu of direct measurements of the Lyman continuum. They obtain the escape fraction after making assumptions about the gas distribution and the relative optical depths in the Lyman continuum and the observed ion (e.g., Heckman et al., 2011).

In this study we use a method that is complementary to other established approaches for investigating the factors that affect the escape of ionizing radiation from starbursts. We map the ionization structure of extended ionized gas in six local starbursts using emission-line ratio images. Spatial changes in emission-line ratios reveal the passage of ionizing radiation through the galaxy (Pellegrini et al., 2012). Thus, by studying the ionization structure of extended ionized gas in our sample, we can determine the optical depth of the galaxies and evaluate the likelihood of escaping Lyman continuum.

\subsection{Method and Data}

\subsubsection{Method}

Determining the fate of ionizing radiation in starburst galaxies is a challenging observational problem. Here, we present an approach that uses the technique of ionization parameter mapping (IPM; Pellegrini et al., 2012). Spatial changes to the ionization

parameter, which evaluates the ionizing photon density relative to the gas density, trace the passage of ionizing radiation through the ISM. In order to track these spatial changes, we create maps of the starbursts using the [SIII]/[SII] line ratio, which acts as a proxy for the ionization parameter. Optically thin and thick regions exhibit different ionization parameter morphologies. In regions that are optically thick, there is a transition from high to low ions at the interface of the ionized region and the neutral environment, whereas optically thin regions lack this transition zone (Pellegrini et al., 2012). Thus, by using ratio maps of two ions, one can distinguish between optically thick and thin regions (Pellegrini et al., 2012). Across an entire galaxy, one can thus use the ionization structure to evaluate whether and in what manner ionizing radiation escapes the galaxy (e.g., Zastrow et al., 2011; Pellegrini et al., 
2012). This method has the advantage that it can be accomplished with narrowband ground-based imaging, which makes it more observationally straightforward than other methods.

\subsubsection{Data}

The galaxies selected for this study are all nearby dwarf starbursts. They have star formation rates $(\mathrm{SFR})$ of $0.05-5 \mathcal{M}_{\odot} / \mathrm{yr}$ and stellar masses $\log \left(\mathrm{M}_{*}\right) \sim 8-10$. Since massive star feedback is thought to play a key role in the escape of Lyman continuum photons (Heckman et al., 2011), we select galaxies that have evidence in the literature for winds and expanding bubbles. Table 3.1 shows the properties of our sample. We calculate the stellar masses (Column 2) using the $K$-band magnitudes from Skrutskie et al. (2003) and the mass to light $(M / L)$ ratios derived from the galaxies' $B-V$ or $B-R$ colors, following (Bell et al., 2003). We restrict this study to starbursts within $45 \mathrm{Mpc}$ to ensure high enough spatial resolution to trace the ionization structure of the extended gas.

We obtained narrow-band emission-line imaging in $\mathrm{H} \alpha$, [SII] $\lambda 6716$, and [SIII] $\lambda 9069$ on the nights of 2009 July 7-11 and 2010 February 12-14. The wavelengths quoted above are the rest frame wavelengths of the emission lines. We imaged the galaxies using the Maryland-Magellan Tunable Filter (MMTF; Veilleux et al., 2010), which is mounted on the Inamori-Magellan Areal Camera and Spectrograph (IMACS) on the Magellan Baade telescope at Las Campanas Observatory. For every emission-line image, we obtained paired continuum exposures of the galaxies. Our observing log is presented in Table 3.2. For each galaxy (Column 1), we list the targeted emission line and central bandpass (Columns 2, 3), exposure times (Columns 4), and the $3 \sigma$ surface brightness limit in the reduced image (Columns 5).

We reduced the MMTF data using version 1.4 of the MMTF data reduction pipeline $^{1}$. This pipeline performs bias and flatfield corrections and then subtracts the sky using an azimuthal averaging. Next, it corrects cosmic rays and bad CCD pixels before mosaicking the different CCD chips into one image. Finally, it averages the

\footnotetext{
${ }^{1}$ http://www.astro.umd.edu/ ${ }^{\sim}$ veilleux/mmtf/datared.html
} 
individual emission-line and continuum images. We flux calibrated the images using standard stars LTT 1020, 6248 and 7987 (Hamuy et al., 1994). We note that for the February 2010 observing run, our measured $\mathrm{H} \alpha$ fluxes are a factor of 2-3 lower than the $\mathrm{H} \alpha$ fluxes reported in the literature. A combination of shallower exposure times, combined with errors in the velocity offsets of our central bandpass, wind activity that shifts flux out the narrow MMTF bandpass, and uncertainty in the [NII] correction factors for the literature values, lead to this offset. However, a comparison with long-slit spectra confirms that the line ratios are sound.

In order to cleanly subtract the continuum from the emission-line images, we first aligned the emission-line and continuum frames using IRAF $^{2}$ tasks wregister and imalign. Next, we used psfmatch to convolve the image with the better seeing with the psf from the other. Finally, we used several bright stars in the field and the task mscimatch to determine the proper scaling to match counts in the stars between the images. We performed this last step iteratively until we obtained a clean continuum subtraction. In $\S 3.3$ we present the final continuum-subtracted $\mathrm{H} \alpha$ images and three-color composite images for each of the galaxies in our sample.

Scattered light in the instrument causes an optical artifact that is not easily corrected for and that stretches across CCD chips 3 and 5. For the most part, we avoid this issue by placing our targets on the other chips. However, for NGC 178, most of our observations have the galaxy sitting on chips 3 or 5 . To mitigate the artifact, we mask the affected region in the flat field. While this increases the flux error on this galaxy, this effect is much less significant than the alteration in flux caused by the artifact on the flat.

We also note that the data from 2009 July observing run are bright time observations. As a result of scattered light in the $\mathrm{f} / 2$ camera and the MMTF instrument, these observations are challenging to reduce. The emission-line images of NGC 178 and NGC 7126 show many background sky features that are caused by these effects (Figure 3.7 and 3.8).

\footnotetext{
${ }^{2}$ IRAF is distributed by NOAO, which is operated by AURA, Inc., under cooperative agreement with the National Science Foundation.
} 
Table 3.1. Sample of Local Starbursts.

\begin{tabular}{|lcccc|}
\hline Galaxy & $\begin{array}{c}\log \left(M_{*}\right)^{a} \\
{\left[\mathcal{M}_{\odot}\right]}\end{array}$ & $\begin{array}{c}\text { Distance } \\
{[\mathrm{Mpc}]}\end{array}$ & $\begin{array}{c}L(\mathrm{H} \alpha) \\
{[\mathrm{erg} \mathrm{s}-1]}\end{array}$ & Ref $^{c}$ \\
\hline NGC 178 & 9.24 & 20.6 & $6.1 \times 10^{40}$ & $(5),(8)$ \\
NGC 1482 & 10.56 & 22.6 & $1.12 \times 10^{42}$ & $(2)$ \\
NGC 1705 & 8.37 & 5.1 & $8.09 \times 10^{39}$ & $(3),(7)$ \\
NGC 7126 & 10.44 & 45.5 & $6.31 \times 10^{41}$ & $(5),(8)$ \\
He 2-10 & 9.50 & 9 & $6.09 \times 10^{40}$ & $(6),(4)$ \\
NGC 3125 & 9.09 & 11.5 & $4.49 \times 10^{40}$ & $(1),(7)$ \\
\hline
\end{tabular}

Note: ${ }^{a}$ Stellar masses calculated using the $M / L$ ratios following (Bell et al., 2003), 2MASS $K$ magnitudes (Skrutskie et al., 2003), and optical colors as follows: B-R colors from Gil de Paz et al. (2003) for NGC 1705 and NGC 3125, B-V colors from Moustakas et al. (2010) for NGC 1482, B-V colors from de Vaucouleurs et al. (1991) for NGC 7126 and NGC 178, B-V colors from Taylor et al. (2005) for NGC 5253, and B-V colors from Ho et al. (2011) for He 2-10. ${ }^{b}$ All distances except that for NGC 1705 are Hubble flow distances for which $H_{0}=70 \mathrm{~km} \mathrm{~s}^{-1} \mathrm{Mpc}^{-1}$

${ }^{c}$ References-(1) Schaerer et al. (1999); (2) Kennicutt et al. (2011);

(3) Tosi et al. (2001); (4) Vacca \& Conti (1992); (5) Meurer et al. (2006);

(6) Johnson et al. (2000); (7) Marlowe et al. (1995); (8) Oey et al. (2007)

\subsection{Results}

In this section we present and discuss the ionization parameter maps of the galaxies individually. In section $\S 3.4$, we discuss the results from the sample as a whole.

\subsubsection{Galaxies with Extended emission}

\section{NGC 5253}

NGC 5253 is a nearby dwarf galaxy currently undergoing an extreme burst of star formation. Using [SIII]/[SII] ratio maps, Zastrow et al. (2011) discovered an optically thin ionization cone in this amorphous elliptical galaxy (Figure 2 of Zastrow et al. (2011)). The ionization cone is narrow with an opening angle $\sim 40^{\circ}$ and is detected out to $\sim 800$ pc from the starburst. The measured opening angle implies a solid angle for the cone that spans $\sim 3 \%$ of $4 \pi$ steradians on the sky, assuming axisymmetry about the cone axis (Zastrow et al., 2011). If many starbursts have similar geometries, this suggests that an orientation bias may limit our ability to detect escaping LyC in 
Table 3.2. Table of Observations

\begin{tabular}{|lcccc|}
\hline $\begin{array}{l}\text { Galaxy } \\
\text { Name }\end{array}$ & band & $\lambda$ & $\begin{array}{c}\text { Exp time } \\
{[\mathrm{s}]}\end{array}$ & $\begin{array}{c}3 \sigma \\
{\left[\mathrm{erg} / \mathrm{s} / \mathrm{cm}^{2} /{ }^{\prime \prime}\right]}\end{array}$ \\
\hline \hline NGC 178 & $\mathrm{H} \alpha$ & 6594 & $3 \times 1200$ & $8.46 \times 10^{-17}$ \\
& {$[\mathrm{SII}]$} & 6749 & $4 \times 1200$ & $1.47 \times 10^{-16}$ \\
& {$[\mathrm{SIII}]$} & 9113 & $5 \times 1200$ & $7.36 \times 10^{-17}$ \\
& & & $1 \times 1800$ & \\
NGC 7126 & $\mathrm{H} \alpha$ & 6628 & $3 \times 1200$ & $9.98 \times 10^{-17}$ \\
& {$[\mathrm{SII}]$} & 6783 & $4 \times 1200$ & $8.25 \times 10^{-17}$ \\
& {$[\mathrm{SIII}]$} & 9159 & $4 \times 1200$ & $4.84 \times 10^{-17}$ \\
\hline & & $12-14 \mathrm{Feb} 2010$ & \\
\hline NGC 1705 & $\mathrm{H} \alpha$ & $6572^{a}$ & $1 \times 1200$ & $9.97 \times 10^{-17}$ \\
& {$[\mathrm{SII}]$} & 6730 & $2 \times 1200$ & $4.10 \times 10^{-17}$ \\
& {$[\mathrm{SIII}]$} & 9087 & $3 \times 1200$ & $6.22 \times 10^{-17}$ \\
He 2-10 & $\mathrm{H} \alpha$ & 6582 & $1 \times 1200$ & $1.33 \times 10^{-17}$ \\
& {$[\mathrm{SII}]$} & 6735 & $3 \times 1200$ & $7.01 \times 10^{-17}$ \\
& {$[\mathrm{SIII}]$} & 9095 & $3 \times 1200$ & $5.82 \times 10^{-17}$ \\
NGC 3125 & $\mathrm{H} \alpha$ & 6587 & $1 \times 1200$ & $1.42 \times 10^{-16}$ \\
& {$[\mathrm{SII}]$} & 6740 & $3 \times 1200$ & $5.02 \times 10^{-17}$ \\
& {$[\mathrm{SIII}]$} & 9102 & $3 \times 1200$ & $3.59 \times 10^{-17}$ \\
NGC 1482 & $\mathrm{H} \alpha$ & 6602 & $1 \times 1200$ & $1.32 \times 10^{-16}$ \\
& {$[\mathrm{SiI}]$} & 6759 & $1 \times 1200$ & $1.20 \times 10^{-16}$ \\
& {$[\mathrm{SIII}]$} & 9121 & $2 \times 1200$ & $5.36 \times 10^{-17}$ \\
\hline
\end{tabular}

Note: ${ }^{a}$ The appropriate wavelength for $\mathrm{H} \alpha$ in NGC 1705 should be $\lambda 6576$. However, our observation set up had the central $\lambda=6572 \AA$ 
starburst and high-redshift galaxies.

\section{NGC 3125}

NGC 3125 is a dwarf galaxy that has a bursty star formation history with roughly three recent epochs of star formation (Vanzi et al., 2011). Two prominent star forming knots, knot A to the northwest and knot B to the southeast (Vacca \& Conti, 1992), both contain massive star clusters and are the main hosts for the Wolf-Rayet population in the galaxy. The ionized gas in NGC 3125 is concentrated around the star-forming knots towards the eastern side of the galaxy relative to the overall stellar distribution (Figure 3.1).
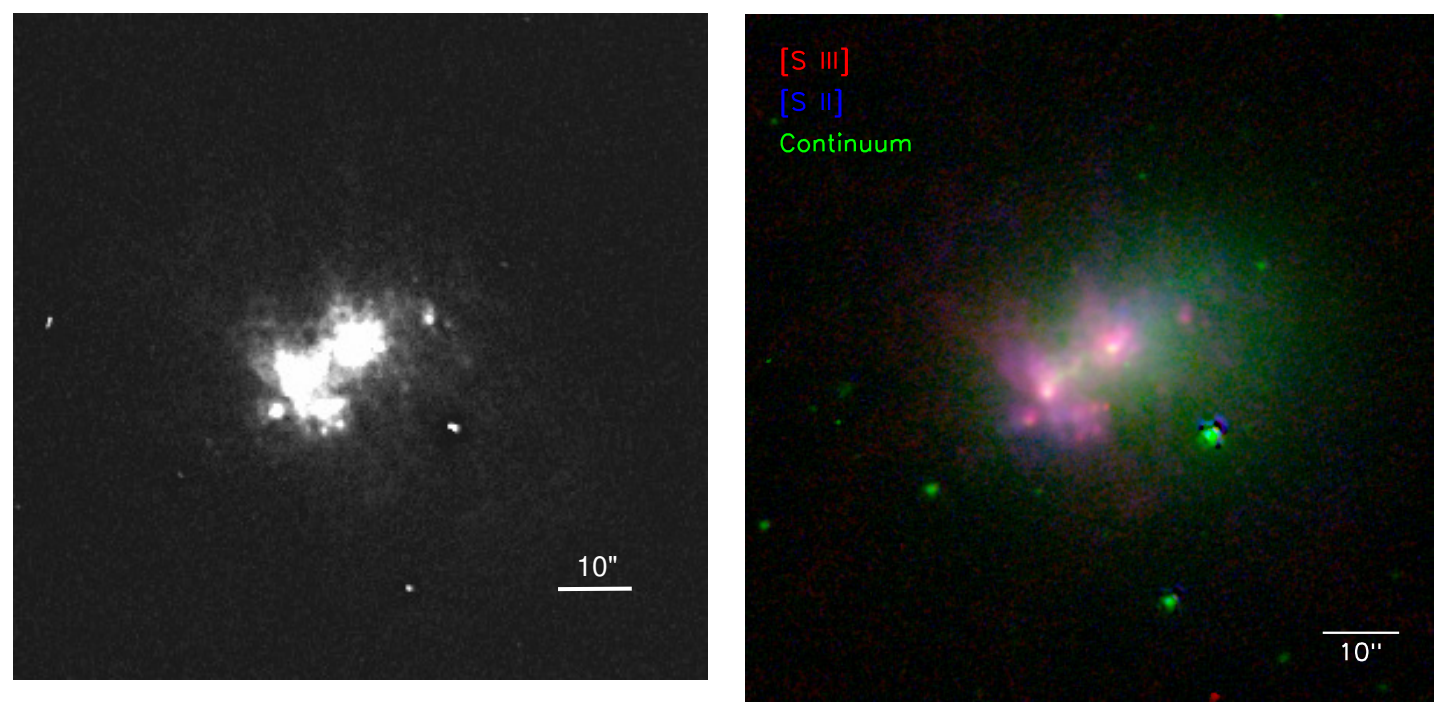

Figure 3.1. Emission-line images of NGC 3125. Left: H $\alpha$ image. The white lines correspond to the ionization cone region used for the red points in Figure 3.2 Right: Three-color composite with $[\mathrm{SiII}] \lambda 9069,[\mathrm{SiI}] \lambda 6716$, and continuum at $\lambda 6680$ in red, blue, and green, respectively. At $11.5 \mathrm{Mpc}$, $10^{\prime \prime}=560 \mathrm{pc}$. In this figure, $\mathrm{N}$ is up and $\mathrm{E}$ is to the left.

We discover a bipolar ionization cone in the emission-line gas extending northeast and southwest of knot B. To the northeast this ionization cone has an opening angle of $55-70^{\circ}$. As can be seen in the ionization parameter map, [SIII]/[SII] (Figure 3.9), the ionization cone to the northeast of knot B exhibits high [SIII]/[SII] throughout. The lack of transition to low values of $[\mathrm{SIII}] /[\mathrm{SII}]$ at the edge implies that the ionization cone is optically thin. This conclusion is supported by the low [SII]/H $\alpha$ observed throughout this portion of the cone (Figure 3.10), which is consistent with expecta- 
tions for optically thin gas (Pellegrini et al., 2012). To the southwest, the line ratios are less conclusive. While they transition to low $[\mathrm{SIII}] /[\mathrm{SII}]$ as one moves away from knot $\mathrm{B}$, there seems to be another transition to high [SIII]/[SII] near the outer edge. However, the line ratios are less certain in this region.

In Figure 3.2, we quantitatively compare the line ratios in the ionization cone to those of the rest of the galaxy. The line ratios are plotted as a function of height above and below the major axis for the ionization cone (red points). The blue points represent the mean of the line ratio from the rest of the galaxy. All points represent pixels that have a minimum $2 \sigma$ detection in all three emission-line bands. From this plot we see that, indeed, there is a clear excess in [SIII]/[SII] and a deficiency of $[\mathrm{SII}] / \mathrm{H} \alpha$ in the NE side of the cone. This behavior is consistent with expectations for optically thin gas.

The trends in the line ratios discussed above are robust, even after considering the effects of internal reddening on the ratio maps. The lines shown on the upper right of Figure 3.2 indicate the changes to the line ratios caused by different levels of reddening. In order to explain the observed $[\mathrm{SIII}] /[\mathrm{SII}]$ excess, the reddening would have to have $A_{V} \sim 5$. We note that the measured reddening toward knots $\mathrm{A}$ and B are $E(B-V)=0.24$ and 0.21 , respectively (Hadfield \& Crowther, 2006), which correspond roughly to $A_{V} \sim 1$. Based on polarimetric observations, most of the dust is located in the plane of the galaxy (Alton et al., 1994). Thus it is reasonable to expect that the extinction in the cone is much less than that toward knots A and B. Furthermore, while strong reddening would enhance the $[\mathrm{SIII}] /[\mathrm{SII}]$ ratio, correcting for it would drive the $[\mathrm{SII}] / \mathrm{H} \alpha$ ratio even lower, which strengthens the argument for optically thin gas.

Marlowe et al. (1995) study the kinematics of the H $\alpha$ gas in NGC 3125 using echelle spectra. They detect a Doppler ellipse along the ionization cone to the northeast, showing that it is a bubble expanding with $v=50 \mathrm{~km} / \mathrm{s}$. This detected bubble is consistent with the idea that massive star feedback clears low-density passages in the ISM through which ionizing radiation may escape. To add further support to this picture, low-resolution X-ray observations show that the X-ray distribution is 
elongated along the minor axis of the galaxy (Fabbiano et al., 1992), suggestive of feedback related activity. Furthermore, the ionization cone occurs at the edge of the galaxy, which may make it easier for ionizing radiation to escape (Gnedin et al., 2008; Paardekooper et al., 2011). The kinematics towards the southwest are less clear, and Marlowe et al. (1995) do not detect a Doppler ellipse in this region.

We also see ionized gas extending to the northeast of knot A. Figure 3.9 shows that while the gas near the cluster has high ionization parameter, it clearly transitions to low ionization parameter as distance from the cluster increases. The transition indicates that the galaxy is optically thick in that direction. The line ratio comparison in Figure 3.2 supports this analysis.
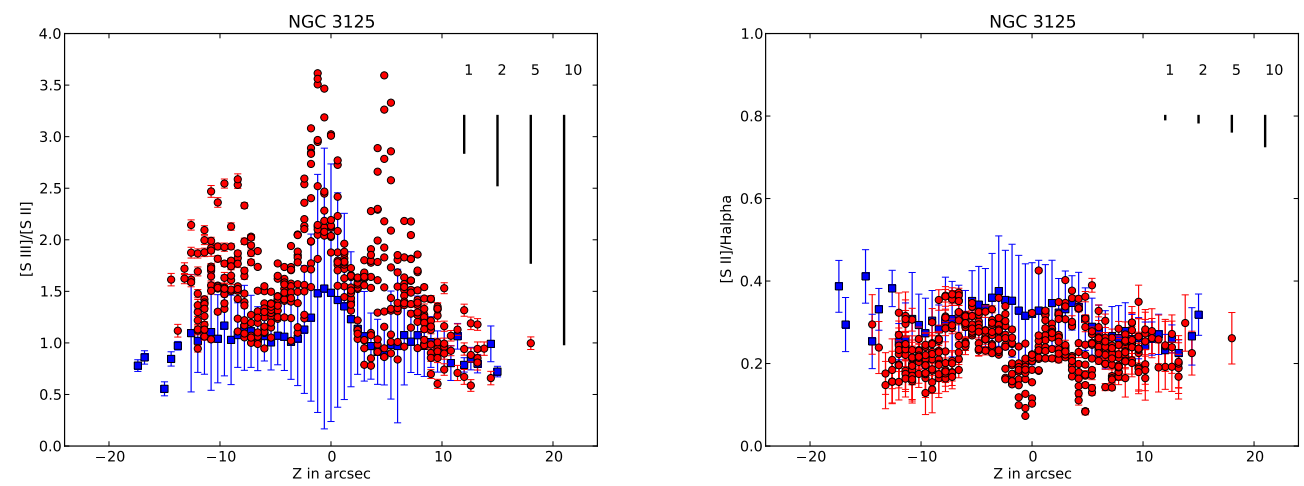

Figure 3.2. Line ratios as a function of height above and below the major axis for the ionization cone (red points) and the mean line ratio from rest of the galaxy (blue points). The cut for the ionization cone is along P.A $\sim 60^{\circ}$. The lines shown on the upper right indicate the change to the line ratios due to reddening of $\mathrm{A}_{V}=1,2,5$, and 10. All points represent pixels that have a minimum $2 \sigma$ detection in all three emission-line bands Negative values are towards the northeast, positive values toward the southwest. This plot demonstrates that there is a clear excess of [SIII]/[SII] and a deficiency of $[\mathrm{SiI}] / \mathrm{H} \alpha$ in the NE side of the cone.

As discussed in $§ 3.2 .2$, our $\mathrm{H} \alpha$ observations are missing a significant amount of flux due to a combination of wavelength calibration errors, velocity offsets, and shallow integration. For this reason, we use the $\mathrm{H} \alpha$ observations of Gil de Paz et al. (2003), to measure the $\mathrm{H} \alpha$ flux in the ionization cone. After correcting for an expected $10 \%$ contribution to the flux from the nearby [NII] lines, we obtain $F(\mathrm{H} \alpha)=2.0 \pm 0.6 \times 10^{-13}$ $\mathrm{erg} \mathrm{s}^{-1} \mathrm{~cm}^{-2}$, which corresponds to a rate of ionizing photons of $\log \left(Q_{0}\right)=51.02$. This means that the equivalent of at least $100( \pm 30)$ O7 stars ionize the cone, assuming $Q_{0}=10^{49}$ for an O7 star at the metallicity of NGC 3125 (Smith et al., 2002). Knot B 
has the equivalent of $1300 \mathrm{O} 7$ stars based on $\mathrm{H} \alpha$ flux (Hadfield \& Crowther, 2006, adjusted to the same $Q_{0}$ per $\mathrm{O} 7$ star). Based on these numbers, roughly $8 \%$ of the ionizing radiation generated by the cluster needs to escape into the NE cone.

The ionization cone in NGC 3125 is narrow and appears somewhat collimated, with an opening angle of $50-75^{\circ}$. This angle corresponds to a solid angle of $5-$ $9 \%$ of $4 \pi$ steradians, assuming axisymmetry about the cone axis. If the cluster emits radiation isotropically, then a geometrical argument requires that 5-9\% of the ionizing radiation from the knot $\mathrm{B}$ escapes. This number agrees with the $\sim 8 \%$ escape fraction based on $\mathrm{H} \alpha$ flux, further linking the ionization cone to the cluster at its base. We note that the $8 \%$ quoted here only refers to the cluster in knot B. Using the total $F(\mathrm{H} \alpha)=3.4 \times 10^{-12} \mathrm{erg} \mathrm{s}^{-1} \mathrm{~cm}^{-2}$ (Marlowe et al., 1995), the cone represents $\sim 6 \%$ of the ionizing flux of the galaxy.

The presence of an optically thin ionization cone suggests that ionizing radiation is escaping this starburst. At the very least, we see that ionizing radiation is escaping the main body of the galaxy and traveling into the halo. The small implied solid angle, in conjunction with a preferred direction is similar to the geometry for NGC 5253, again supporting the possibility that an orientation bias affects our ability to detect escaping Lyman continuum in starbursts and LBGs.

\section{NGC 1705}

NGC 1705 is a nearby starburst galaxy with a stunning starburst-driven galactic wind (Meurer et al., 1992). NGC 1705 has a combined stellar and gas mass of $\sim 2.7 \times 10^{8} \mathcal{M}_{\odot}$ (Meurer et al., 1992). The metallicity measurements range from $12+\log (\mathrm{O} / \mathrm{H})=8.21$ to 8.46 (Lee \& Skillman, 2004; Storchi-Bergmann et al., 1994; Meurer et al., 1992). Like similar dwarf starbursts, NGC 1705 has a bursty star formation history with two recent star forming epochs in the last 15 Myr (Annibali et al., 2003, 2009). One of these bursts formed the well known super-star cluster (SSC) at the center, which has $\log (M) \sim 10^{5}$ and an age of $\sim 10$ Myr (Melnick et al., 1985; O’Connell et al., 1994; Ho \& Filippenko, 1996). This SSC generates most of the UV luminosity and is consistent with a population dominated by B stars (Heckman 
\& Leitherer, 1997). That same 10-15 Myr old burst of star formation is thought to have launched the galactic wind (Annibali et al., 2003, 2009). This burst was followed by a more recent epoch of star formation, $\sim 3$ Myr ago that may have been triggered by the wind (Annibali et al., 2003, 2009).
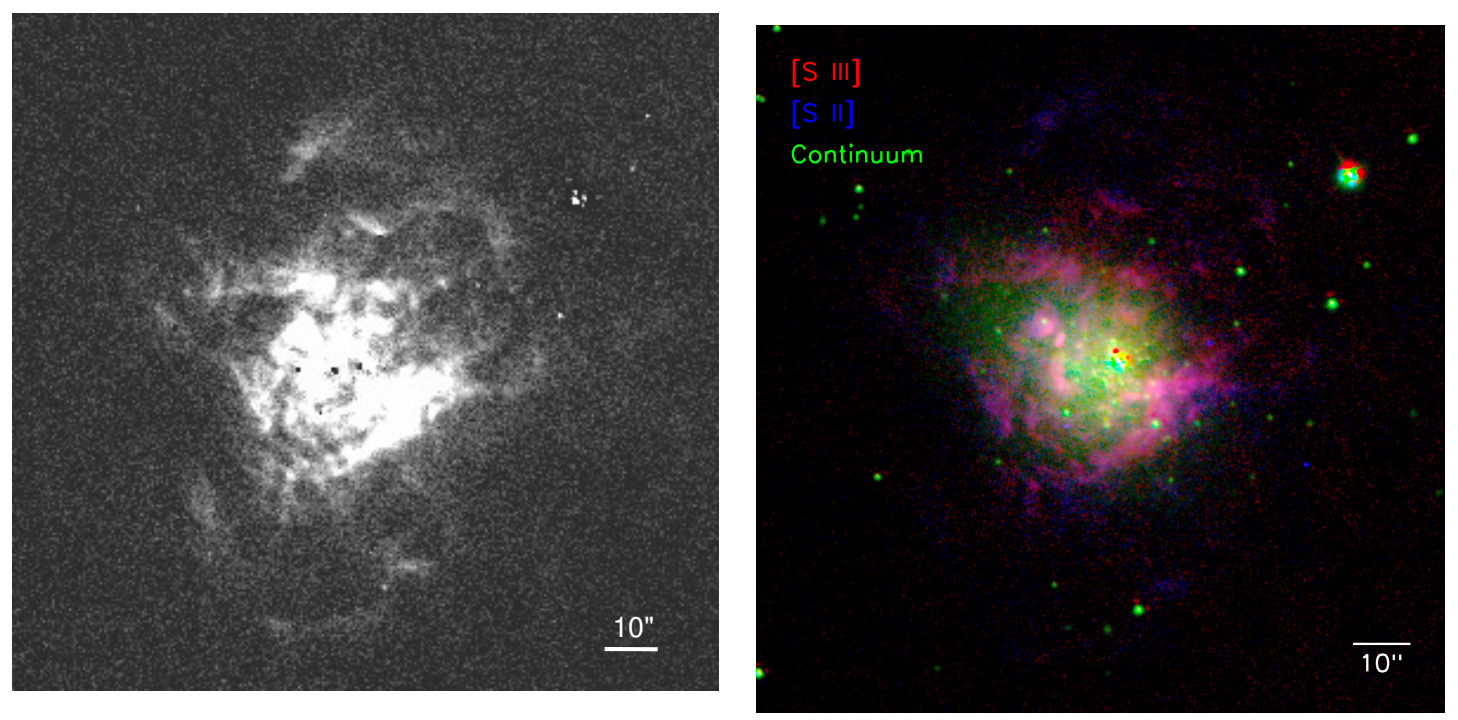

Figure 3.3. Emission-line images of NGC 1705. Left: $\mathrm{H} \alpha$ Right: Three-color composite with [SIII] $\lambda 9069,[\mathrm{SiI}] \lambda 6716$, and continuum at $\lambda 9180$ in red, blue, and green, respectively. At $5.1 \mathrm{Mpc}$, $10^{\prime \prime}=250$ pc. In this figure, $\mathrm{N}$ is up and $\mathrm{E}$ is to the left.

In emission-line gas, NGC 1705 shows filamentary extended substructure as a result of its galactic wind (Meurer et al., 1992). Based on $\mathrm{H} \alpha$ observations, the superbubble has an expansion velocity 50-132 km/s (Marlowe et al., 1995; Meurer et al., 1992). Heckman \& Leitherer (1997) studied the kinematics using UV interstellar absorption lines and found further evidence for an expanding wind. X-ray observations show that the wind has similar size and morphology to the optically detected gas (Strickland et al., 2004). The star-forming nucleus resides on the axis of symmetry for the superbubble, which further supports that the wind is driven by star formation (Meurer et al., 1992). Shocks contribute only a small amount to the ionization in the wind, as most of the emission-line gas is photo-ionized (Marlowe et al., 1995; Veilleux et al., 2003). Our emission-line images and ratio maps for NGC 1705 are shown in Figures 3.3, 3.9,3.10, and 3.11. The ionized gas associated with the wind is clearly detected in both [SIII] and [SII]. 
The spatial changes in the [SIII]/[SII] ratio map suggest that the wind in NGC 1705 is optically thick. Close to the starburst, the $[\mathrm{SIII}] /[\mathrm{SII}]$ ratio is high, as expected from photoionized gas near the ionization source. However, as one looks further into the wind, there is a clear transition towards lower $[\mathrm{SIII}] /[\mathrm{SII}]$ ratios, and at the far edge of the shells, the line ratios indicate the [SII] emission is dominant. This is further supported by the $[\mathrm{SII}] / \mathrm{H} \alpha$ and $[\mathrm{SIII}] / \mathrm{H} \alpha$ ratio maps in Figures 3.10 and 3.11. In these maps, the $[\mathrm{SII}] / \mathrm{H} \alpha$ ratio increases at the edges of the filament, and, as expected based on Figure 3.9, the $[\mathrm{SIII}] / \mathrm{H} \alpha$ ratio decreases. While this behavior is consistent with optically thick gas, we note that if the radiation field is dominated by late type stars, as in the case of NGC 1705, the gas may simultaneously exhibit a low-ionization transition zone and be optically thin (Pellegrini et al., 2012).

In addition to evaluating the optical depth in the plane of the sky, the ratio maps can be used to evaluate the optical depth along the line of sight. For gas with abundances similar to that of the LMC, $[\mathrm{SII}] / \mathrm{H} \alpha<0.05$ is a diagnostic for optically thin photoionized gas along the line of sight. Of particular interest is the region around NGC 1705-1, the super star cluster. Heckman \& Leitherer (1997) estimated a very low absorbing HI column, $N_{\mathrm{HI}}=1.5 \times 10^{20} \mathrm{~cm}^{-2}$, toward NGC 1705-1, the super star cluster at the center of the starburst. This column is an order of magnitude smaller than the column measured from $21 \mathrm{~cm}$ observations, which suggests that the cluster is either sitting in front of most of the HI or that we are observing the SSC through a hole in the ISM (Heckman \& Leitherer, 1997). Either scenario increases the likelihood for escaping Lyman continuum. However, Figure 3.10 shows that $[\mathrm{SII}] / \mathrm{H} \alpha$ $>0.05$ over the entire nuclear region, which implies that even though the neutral gas is patchy, the gas is optically thick.

It is expected that strong starburst feedback, such as that present in NGC 1705, is conducive to escaping Lyman continuum. However, our observations indicate that the wind in NGC 1705 is optically thick. Both the strength of the radiation field and the amount of gas available to absorb the ionizing radiation will determine whether the galactic wind is optically thick or thin. While there have been two recent epochs of star formation, one 10-15 Myr ago and one $~ 3$ Myr ago (Annibali et al., 2003, 
2009), the ionizing population is dominated by B stars (Heckman \& Leitherer, 1997).

This means that there are relatively fewer ionizing photons being emitted and the radiation field is softer than if there were significant numbers of $\mathrm{O}$ stars. In addition to a reduced radiation field, a s̀pur' of HI gas is associated with the outflow (Meurer et al., 1998). This spur of neutral gas is likely co-spatial with the optically detected galactic wind based on its kinematics and orientation (Meurer et al., 1998; Elson et al., 2013). The feature has an HI column density of $\sim 10^{20} \mathrm{~cm}^{-2}$, which is more than high enough to be optically thick to Lyman continuum. Thus, despite the presence of a strong galactic wind, NGC 1705 is likely optically thick due to a combination of population age and gas morphology.

\section{He 2-10}

He 2-10 is one of the prototypical HII galaxies (Allen et al., 1976). It is $9 \mathrm{Mpc}$ away (Vacca \& Conti, 1992) and has dynamical mass $\sim 3 \times 10^{9} \mathcal{M}_{\odot}$ (Kobulnicky et al., 1995). In emission-line gas there are many notable loops and filaments (Figure 3.4) whose kinematics suggest expanding bubbles and possible outflows (Méndez et al., 1999). These bubbles are centered on sites of recent star formation. He 2-10 is the host of nearly 80 SSCs (Johnson et al., 2000). The central starburst contains a handful of clusters in ultra-compact HiI regions that contain the ionizing equivalent of 700-2600 O7 stars each (Johnson \& Kobulnicky, 2003). In addition to prodigiously forming stars, SFR $0.87-2 \mathcal{M}_{\odot} /$ yr (Calzetti et al., 2010; Reines et al., 2011), this galaxy was recently found to be the host of an AGN (Reines et al., 2011).

In the ratio map, the central star forming region stands out with high [SIII]/[SII], as would be expected for a region hosting thousands of $\mathrm{O}$ stars. As the radius from the center increases, $[\mathrm{SIII}] /[\mathrm{SII}]$ drops considerably. There is a local maximum $\sim 8^{\prime \prime}$ to the east around the location of the second SSC. However, even here the emissionline gas transitions to low $[\mathrm{SIII}] /[\mathrm{SII}]$ toward the edge of the expanding bubble. The contrast at the edges of the bubbles is seen more clearly in the $[\mathrm{SII}] / \mathrm{H} \alpha$ ratio maps, where a clear enhancement of $[\mathrm{SII}] / \mathrm{H} \alpha$ is present. In contrast the $[\mathrm{SIII}] / \mathrm{H} \alpha$ falls off as the distance from the ionizing source increases, as would be expected for an optically 

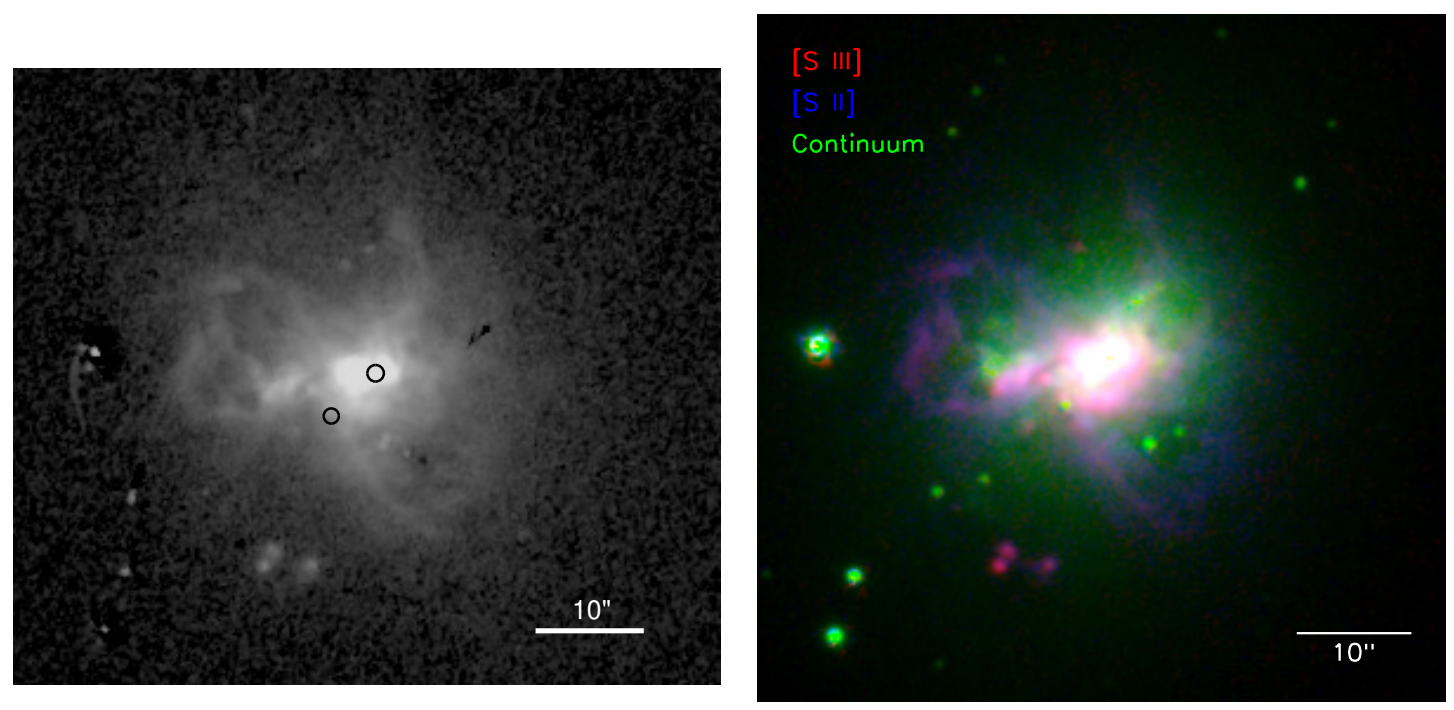

Figure 3.4. Emission-line images of He 2-10. Left: $\mathrm{H} \alpha$ image. The two circles correspond to locations that have $[\mathrm{SII}] / \mathrm{H} \alpha<0.05$. Right: Three-color composite with $[\mathrm{SIII}] \lambda 9069$, [Sir] $\lambda 6716$, and continuum at $\lambda 6680$ in red, blue, and green, respectively. At $9 \mathrm{Mpc}, 10^{\prime \prime}=460 \mathrm{pc}$. In this figure, $\mathrm{N}$ is up and $\mathrm{E}$ is to the left.

thick nebula.

The spatial changes in the line ratio maps are consistent with expanding superbubbles. High ionization parameter gas is present close to the ionizing source, while the edges of the bubbles have low ionization parameter and possible contribution to [SII] from shocks. Studies of the $\mathrm{H} \alpha$ kinematics indicate a potential Doppler ellipse in the NE bubble and velocities differences of up to $\pm 300 \mathrm{~km} / \mathrm{s}$ across the galaxy (Méndez et al., 1999). Using UV interstellar lines, Johnson et al. (2000) determined that the bulk motion of the ISM indicates an outflow with $v \sim 360 \mathrm{~km} / \mathrm{s}$. The velocities reported by both works are in excess of the escape velocity, $\sim 160 \pm 30 \mathrm{~km} / \mathrm{s}$ (Johnson et al., 2000). However, the closed loops of the outflow seen in the $\mathrm{H} \alpha$ images suggest that these bubbles have not yet broken out of the ISM to form a galactic fountain. Bubble walls severely hinder the passage of ionizing radiation, and can delay the escape of Lyman continuum radiation until $Q_{0}$ has dropped considerably (Dove et al., 2000; Fujita et al., 2003).

He 2-10 is unique in our sample, in that it is the only galaxy that contains a confirmed AGN. This AGN was discovered by Reines et al. (2011) as a radio and X-ray point source and is located near the star forming region of knot $\mathrm{A}$. The accretion rate 
onto the AGN is modest, but may have been greater in the past (Reines et al., 2011). Thus, the presumed tracers for massive-star feedback, such as the large expanding bubbles, may be contaminated by contributions from the AGN as opposed to being driven by star formation.

The distribution of gas in the ISM plays a critical role in the passage of ionizing radiation in a galaxy. The HI observations from Kobulnicky et al. (1995) show that the neutral ISM extends significantly beyond the optically detected ionized gas. This observation is consistent with the observations of optically thick bubbles. However, if this overall HI envelope has a clumpy distribution, it is still possible to have small low-density passages through which ionizing radiation may escape the starburst. The $[\mathrm{SII}] / \mathrm{H} \alpha$ ratio map shows two locations that have $[\mathrm{SII}] / \mathrm{H} \alpha<0.05$ and may be optically thin. We note these locations with small circles on Figure 3.4. One of these regions coincides with the location of the AGN, and the other lies $4^{\prime \prime}$ to the SE from the first. While our data cannot establish the presence of an optically thin path from the galaxy conclusively, these observations are suggestive that feedback from the AGN or star formation in the region have cleared out a small hole in the ISM. The variable reddening measurements derived from different wavelengths and positions in the galaxy further suggest an inhomogeneous distribution of gas and dust throughout the galaxy (e.g., Sauvage et al., 1997). The presence of small local holes is also supported by the fact that many of the probably young SSCs do not show commensurate $\mathrm{H} \alpha$ emission, which suggests that gas has been evacuated in the regions of these clusters (Johnson et al., 2000). If this is the case, it supports the picture that ionizing radiation escapes galaxies through small holes and that an orientation bias affects our ability to detect escaping Lyman continuum. We note that these line ratios have not been corrected for reddening. However, any reddening correction would drive the $[\mathrm{SII}] / \mathrm{H} \alpha$ line ratio lower, strengthening the argument for optically thin gas along the line of sight.

\section{NGC 1482}

NGC 1482 is an SA0 (de Vaucouleurs et al., 1991) with a well known galactic wind (Hameed \& Devereux, 1999). At a distance of 22.6 Mpc (Kennicutt et al., 2011), 
this starburst is a highly inclined disk with a prominent dust lane (de Vaucouleurs et al., 1991). The wind was first discovered by (Hameed \& Devereux, 1999) in optical emission-lines, with later optical and X-ray observations confirming its nature as a wind (Veilleux \& Rupke, 2002; Strickland et al., 2004).
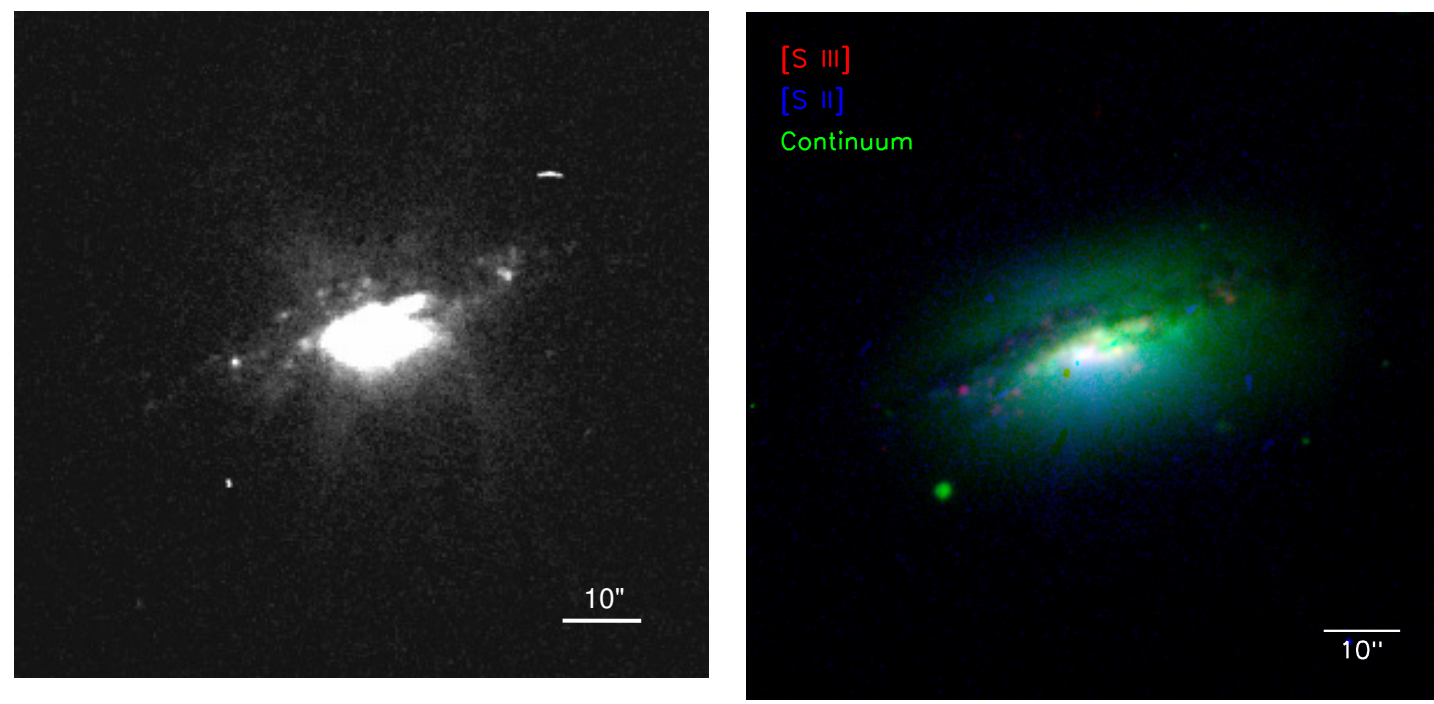

Figure 3.5. Emission-line images of NGC 1482. Left: $\mathrm{H} \alpha$ Right: Three-color composite with [SIII] $\lambda 9069,[\mathrm{SII}] \lambda 6716$, and continuum at $\lambda 6680$ in red, blue, and green, respectively. At $22.6 \mathrm{Mpc}$, $10^{\prime \prime}=1.1 \mathrm{kpc}$. In this figure, $\mathrm{N}$ is up and $\mathrm{E}$ is to the left.

In our emission-line images (Figure 3.5), we primarily detect the wind in [SII] and $\mathrm{H} \alpha$. The [SIII] emission is confined to the star forming region in the inner disk. The ionization parameter map shows strong evidence for radiation bounding in NGC 1482 . High $[\mathrm{SIII}] /[\mathrm{SII}]$ traces the disk star forming region, and the line ratio drops precipitously as one moves further into the wind. Veilleux et al. (2003) found the wind to be dominated by shock ionization based on $[\mathrm{NII}] / \mathrm{H} \alpha$ observations. Our $[\mathrm{SII}] / \mathrm{H} \alpha$ map (Figure 3.10) confirms this picture with $[\mathrm{SII}] / \mathrm{H} \alpha \geq 0.50$ in the wind.

In reality, the emission observed in the wind will be a combination of shock- and photoionized gas. In order to put strong constraints on the [SIII]/[SII] line ratio in the wind, we explore correcting the line ratios for the shock ionization as follows. As discussed by Jaskot \& Oey (2013), the observed line ratio can be written as:

$$
\frac{A_{o}}{B_{o}}=\frac{A_{s}+A_{p}}{B_{s}+B_{p}}
$$


where $\mathrm{A}$ and $\mathrm{B}$ correspond to the emission lines in question, and the subscripts $o, s$, and $p$ indicate the observed, shocked, and photoionized line strengths, respectively. To convert our observed line ratios to the line ratios of photoionized gas, we need to solve for the fraction of shock-ionized relative to photoionized gas,

$$
X=\frac{H_{s}}{H_{p}}
$$

Together with Equation 3.1, this yields:

$$
\frac{A_{p}}{B_{p}}=\frac{A_{o}}{B_{o}}+X\left(\frac{A_{o}}{B_{o}}-\frac{A_{s}}{B_{s}}\right)
$$

In Equation 3.3, $\frac{A_{s}}{B_{s}}$ can be replaced by the predicted line strengths from MAPPINGS III shock models (Allen et al., 2008). Since Veilleux \& Rupke (2002) observed velocities of up to $v=250 \mathrm{~km} / \mathrm{s}$ in the wind, we explore shocks with $v \leq 950 \mathrm{~km} / \mathrm{s}$ (assuming strong shocks). For this calculation we use the MAPPINGS III shock-only models, which provide the most optimistic correction factor for high [SIII]/[SII] from photoionization. The precursor component to the shock would increase the relative contribution of shocks to the [SIII], thus moving the corrected line ratios more towards optically thick than the shock only models. To solve for $X$, we write Equation 3.3 for each emission line ratio, $[\mathrm{SIII}] /[\mathrm{SII}],[\mathrm{SIII}] / \mathrm{H} \alpha$, and $[\mathrm{SII}] / \mathrm{H} \alpha$. By combining the resultant equations, we finally solve for $X$ :

$$
X=\frac{D_{1}\left(\frac{[S I I]}{H \alpha}_{o}\right)+D_{2}\left(\frac{[S I I I]}{[S I I]}{ }_{o}\right)+D_{3}}{D_{3} D_{2}}
$$

where

$$
D_{1}=\frac{[\mathrm{SIII}]}{[\mathrm{SII}]_{\mathrm{o}}}-\frac{[\mathrm{SIII}]}{[\mathrm{SII}]_{\mathrm{s}}}
$$

and $D_{2}$ and $D_{3}$ are the corresponding values for $[\mathrm{SII}] / \mathrm{H} \alpha$ and $[\mathrm{SIII}] / \mathrm{H} \alpha$, respectively. After solving for $X$, we use that value in Equation 3.3 to create a new [SIII]/[SII] map (Figure 3.6). We find that the $[\mathrm{SIII}] /[\mathrm{SII}]$ maps remain strongly radiation bounded. 


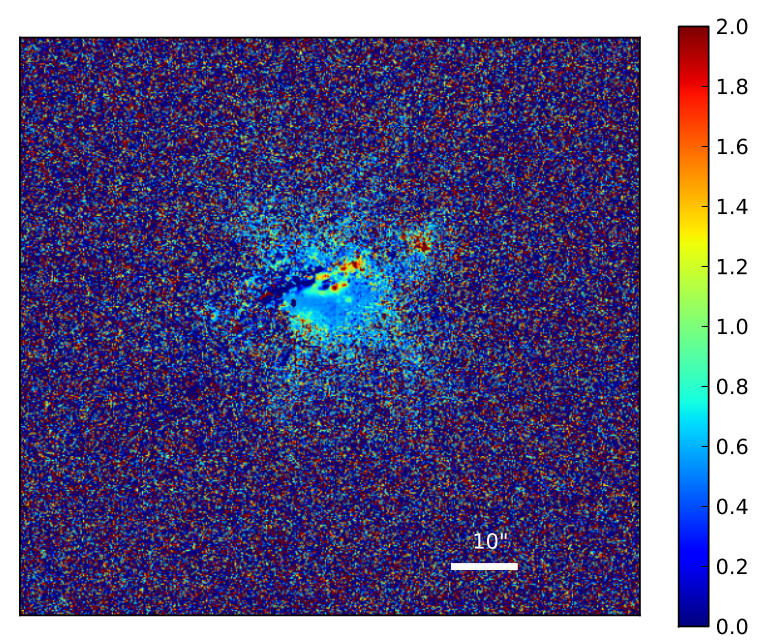

Figure 3.6. Shock-corrected ionization parameter map of [SIII]/[SII]. This map is created assuming shock velocities of $950 \mathrm{~km} / \mathrm{s}$ and the MAPPINGS III shock-only model (Allen et al., 2008). Even in the most optimistic correction, the $[\mathrm{SIII}] /[\mathrm{SII}]$ ratio transitions to values $<0.70$ close to the disk of the galaxy. $\mathrm{N}$ is up and $\mathrm{E}$ is to the left.

Since there is evidence that the wind has cleared gas from the ISM of NGC 1482, it is somewhat surprising that this object does not show evidence for an optically thin wind. HI observations reveal a hole in the HI distribution at the location of the wind (Hota \& Saikia, 2005; Omar \& Dwarakanath, 2005). Further evidence that the wind has cleared material from the center of the galaxy is found by Sharp \& Bland-Hawthorn (2010). They generate a $[\mathrm{SII}] \lambda 6716 / \lambda 6732$ ratio map and see a sharp gradient at the base of the wind, which suggests clearing of the inner disk by the wind (Sharp \& Bland-Hawthorn, 2010). Both of these lines of evidence suggest that ionizing radiation can escape along the wind. Perhaps the massive star population in NGC 1482 has aged in the time since the wind launched, and, thus, $Q_{0}$ has dropped significantly. Another explanation could be that dust entrained in the wind (Vagshette et al., 2012) provides additional material that absorbs radiation as it travels away from the galaxy.

We note here that some of the emission may be shifted out of our very narrow bandpasses. For $v \sim 250 \mathrm{~km} / \mathrm{s}$, the emission will shift by $5 \AA$ and $7 \AA$ for [SII] and [SIII], respectively. This is comparable to half the bandpass in each of those filters. 
Exacerbating this effect, our central bandpass in [SIII] is shifted $\sim 4 \AA$ from the appropriate central bandpass for the redshift of NGC 1482. This means that for NGC 1482 we do not detect some of the redshifted emission from [SIII] in the wind, although we should detect all of the blueshifted component. That being said, the wind exhibits the transition from high to low ionization parameter gas relatively close to the disk of the galaxy. Furthermore, the $[\mathrm{SIII}] /[\mathrm{SII}]$ line ratio is well below 0.5 for most of the wind. Even if we assume that we are missing half of the [SIII] flux in the wind because of the velocity offset, the difference does not push the [SIII]/[SII] ratio high enough to match our expectations for optically thin emission. Given the $5 \mathrm{kpc}$ extent of the wind (Strickland et al., 2004), the location of the transition zone makes it less likely that the transition is a spurious one.

\subsubsection{Non-Detections}

\section{NGC 178}

NGC 178 is forming stars at a rate of $0.549 \mathcal{M}_{\odot} / y r$ (Oey et al., 2007) and lies 20.6 Mpc away (Meurer et al., 2006). In the emission-line images we see that ionized gas is split between two areas, one around the bulk of the observed continuum, and a separate extra-planar region (Figure 3.7). Since ionizing radiation emitted at the edge of galaxies is more likely to escape (Gnedin et al., 2008), it might be reasonable to expect to find some evidence of density bounding in this region of the ionization parameter map.

However, the ionization parameter map shows that all nebular regions are optically thick. The ionized gas to the west is comprised of a few separate peaks in the $[\mathrm{SIII}] /[\mathrm{SII}]$ ratio map (Figure 3.9), all of which exhibit a clear transition to low ionization parameter gas at the edges.

The $[\mathrm{SII}] / \mathrm{H} \alpha$ map (Figure 3.10) is intriguing. There appears to be an envelope of high $[\mathrm{SII}] / \mathrm{H} \alpha$ surrounding the galaxy. This envelope could be caused by the well known $[\mathrm{Sir}] / \mathrm{H} \alpha$ gradient in the warm, ionized medium of galaxies (Rand, 1998) or could be a signature of shocked gas surrounding the galaxy. We note, however, that there are significant sky gradients and background features around the galaxy that 

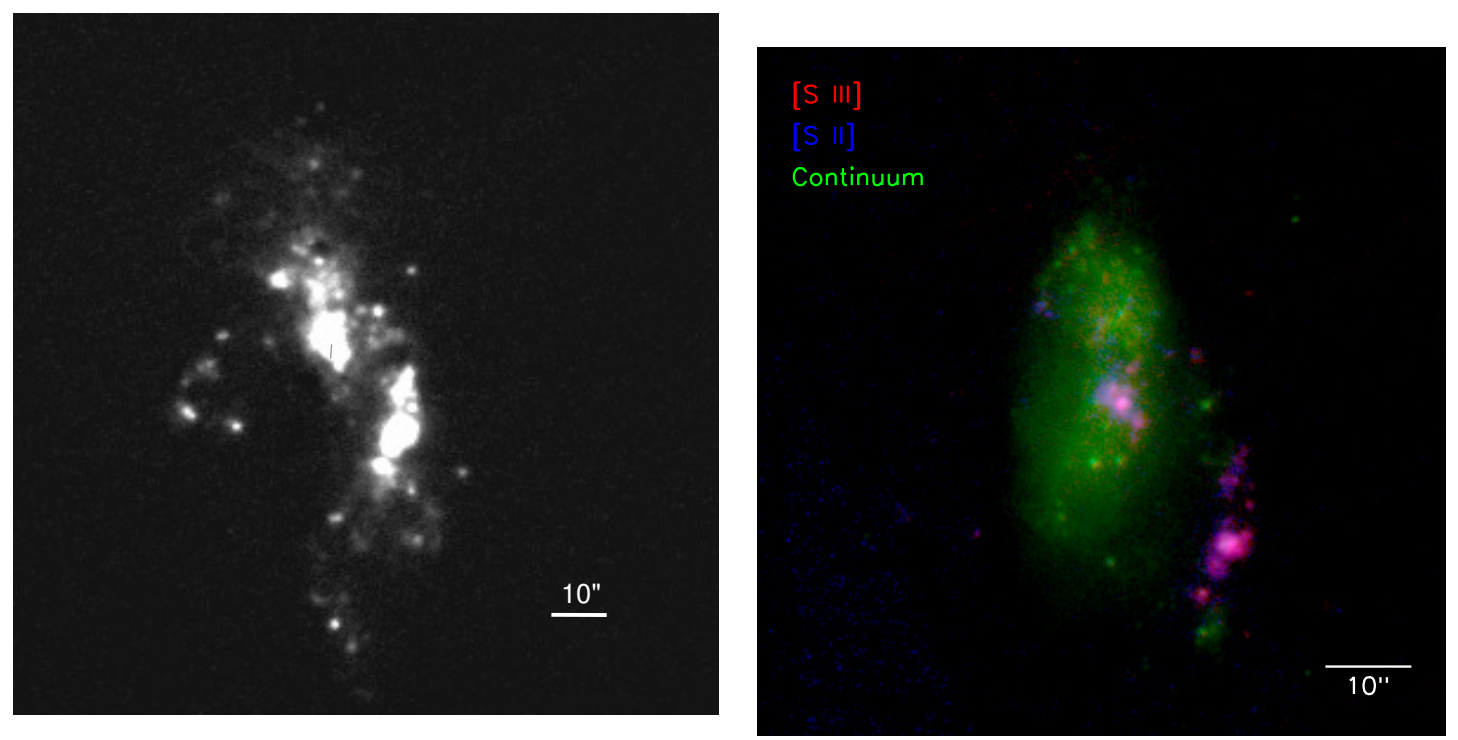

Figure 3.7. Emission-line images of NGC 178. Left: $\mathrm{H} \alpha$ Right: Three-color composite with [SIII] $\lambda 9069$, [SII] $\lambda 6716$, and continuum at $\lambda 6680$ in red, blue, and green, respectively. At $20.6 \mathrm{Mpc}$, $10^{\prime \prime}=1 \mathrm{kpc}$

are caused by scattered light within the instrument and by bright time observing conditions (§3.2.2). These sky features make it challenging to determine the nature of the surrounding low ionization parameter envelope.

\section{NGC 7126}

NGC 7126 is an SA(rs)c galaxy (de Vaucouleurs et al., 1991). At $45.5 \mathrm{Mpc}$ away (Meurer et al., 2006), it is the most distant galaxy in our sample. The emission line images reveal an extended spiral structure filled with Hiı regions (Figure 3.8). HI observations show that it is interacting with its nearby companion NGC 7125 (Nordgren et al., 1997). NGC 7126 does not have a catalogued wind, and, with its high star formation rate, acts as a control in the sample.

The $[\mathrm{SIII}] /[\mathrm{SII}]$ map shows a remarkably low ratio of $[\mathrm{SIII}] /[\mathrm{SII}]$. While the spiral structure is detected, it does not show up as clearly as it does in the $\mathrm{H} \alpha$ ratio maps. In the $[\mathrm{SII}] / \mathrm{H} \alpha$ ratio map, the HII regions themselves appear with line ratios consistent with photoionized gas. As one moves to the edges of the individual regions, $[\mathrm{SII}] / \mathrm{H} \alpha$ increases. This is consistent with expectations of the warm, ionized medium in galaxies (Rand, 1998). We note, however, that these line ratios are a bit high, and 

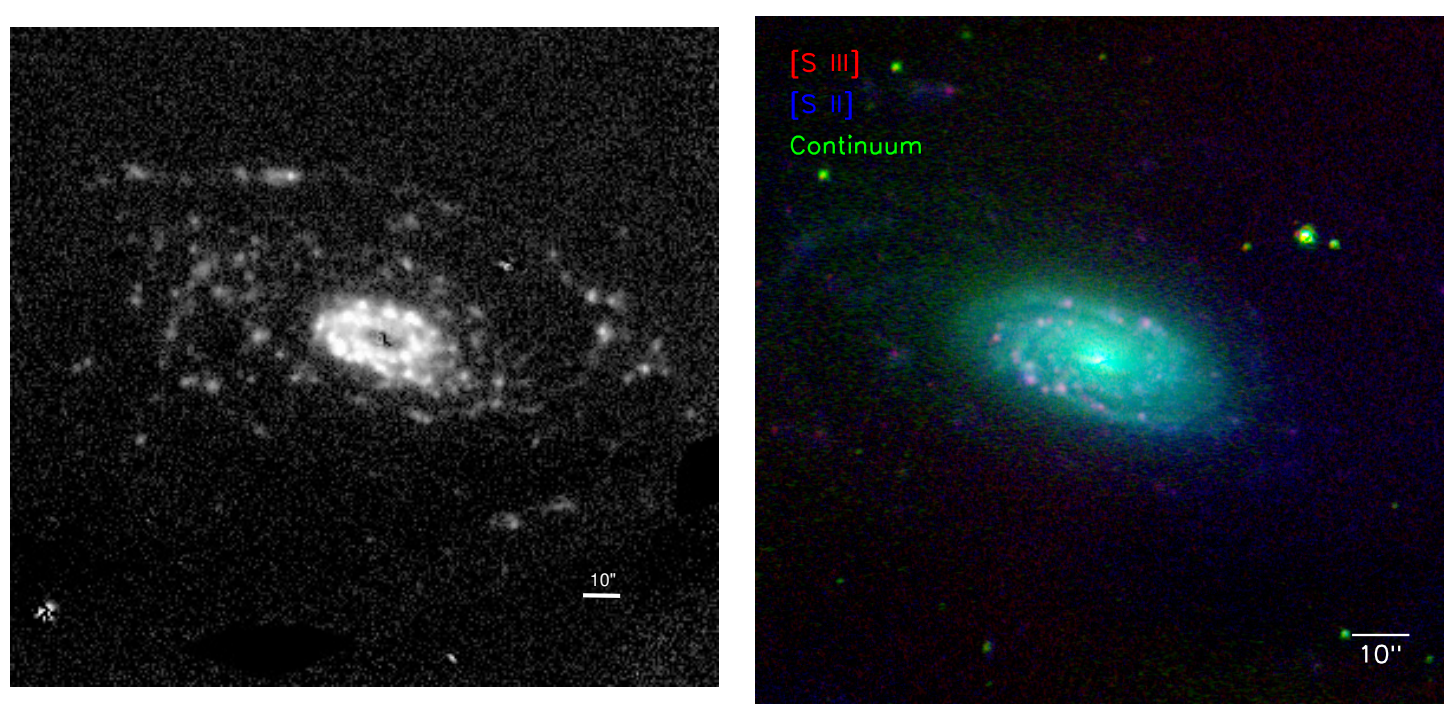

Figure 3.8. Emission-line images of NGC 7126. Left: $\mathrm{H} \alpha$ Right: Three-color composite with [SIII] $\lambda 9069$, [SiI] $\lambda 6716$, and continuum at $\lambda 6680$ in red, blue, and green, respectively. At $45.5 \mathrm{Mpc}$, $10^{\prime \prime}=2.3 \mathrm{kpc}$. In this figure, $\mathrm{N}$ is up and $\mathrm{E}$ is to the left.

they approach values consistent with shock ionization. The $[\mathrm{SIII}] / \mathrm{H} \alpha$ map exhibits relatively low line ratios throughout, even at the centers of the observed Hir regions. This could be indicative that the ionizing stellar population is composed primarily of $\mathrm{B}$ or late $\mathrm{O}$ type stars.

\subsection{Discussion}

The full sample of seven galaxies provides examples of both optically thin and optically thick extended emission-line gas. By comparing the properties of these galaxies we can gain insight on the processes and properties that influence the escape of ionizing radiation.

\subsubsection{Orientation bias}

The interpretation of escaping Lyman continuum observations in starbursts and LBGs is challenging because of the need to disentangle observational effects from intrinsic trends. One issue under debate is whether the relatively few $(\sim 10 \%)$ galaxies that have significant $f_{\text {esc }}$ are representative of the fraction of galaxies that have escaping Lyman continuum. Potential challenges that can modify the intrinsic distribution of $f_{\text {esc }}$ include low redshift interlopers, a preferred direction of escape, and small covering 
fractions for the escaping Lyman continuum (e.g., Vanzella et al., 2012; Nestor et al., 2011). Examining the morphologies of the ionization cones observed in this work can shed light on this issue.

Two of the seven starburst galaxies studied here show strong evidence for optically thin ionization cones. In both NGC 5253 (see also Zastrow et al., 2011) and NGC 3125, the cones are aligned along the minor axis. This behavior is consistent with the expectations of massive-star feedback-driven models (e.g., Murray et al., 2011; Kim et al., 2012). The observed alignment implies a preferred direction; feedback will punch holes in the ISM more easily along the minor axis (e.g., Veilleux et al., 2005). In a study of low redshift LBG analogs, Heckman et al. (2011) measured extreme wind velocities $(v \sim 1500 \mathrm{~km} / \mathrm{s})$ in all galaxies with low optical depth to Lyman continuum. The high velocities indicate the direction of the wind must be nearly aligned with the line of sight to the galaxy. This relationship between low optical depth and high outflow velocities further strengthens the argument that Lyman continuum escape has a preferred direction (Zastrow et al., 2011). Furthermore, the ionization cones exhibit narrow morphologies and subtend an estimated $4-10 \%$ of $4 \pi$ steradians. This implies that the covering fraction of escaping radiation will be much less than unity, and is consistent with the results from studies of high redshift star-forming galaxies (Nestor et al., 2011) and similar studies of ionization cones in quasars (e.g., Kreimeyer \& Veilleux, 2013). We note that the narrow ionization cones do not necessarily imply small $f_{\text {esc }}$. If the physical size of the cone's base is significantly larger than the size of the ionizing cluster, then large fractions of the ionizing flux produced by the cluster may still escape through the angularly narrow cone. The combination of small opening angles and preferred directions suggestd that if one tried to directly detect escaping Lyman continuum, the axis of escape would need to be close to the observer's line of sight in order to obtain a strong detection. If more galaxies are like NGC 3125 and NGC 5253, then an orientation bias makes it more challenging to detect escaping Lyman continuum in starbursts and LBGs. 


\subsubsection{Possible Trends with Galaxy Properties}

In light of the massive-star feedback-driven model discussed previously, the first galactic property to examine is the presence of a galactic wind. All of the galaxies that show evidence for extended emission also have evidence for either a galactic wind or superbubble expansion out of the galaxy. Thus confirming the link between winds and the fate of ionizing radiation (e.g., Heckman et al., 2011). However, NGC 1705 and NGC 1482, the two galaxies that have the most clearly established winds, both appear optically thick. We note that both galaxies without extended emission, NGC 178 and NGC 7126, do not have galactic winds. Thus, as discussed in Heckman \& Leitherer (1997), we see that while wind activity is important for the escape of Lyman continuum, other factors clearly play a determining role.

Another critical property to examine is star formation rate. Models predict that escaping ionizing radiation will be associated with extreme feedback from strongly star forming systems (e.g., Clarke \& Oey, 2002; Wise \& Cen, 2009; Paardekooper et al., 2011). Table 3.3 presents the star forming properties for each galaxy in the sample. Columns 1 and 2 are the galaxy name and HI mass, respectively; Columns 3,4 , and 5 present the $\mathrm{H} \alpha$ derived SFR, SFR surface density $\left(\Sigma_{\mathrm{SFR}}\right)$, and the specific SFR (sSFR), respectively. Column 6 provides the references for the star formation rates, $\mathrm{HI}$ masses and $\mathrm{H} \alpha$ radii. Finally, Column 7 indicates whether the galaxy shows wind and/or expanding bubble activity, and Column 8 shows the ratio of HI mass to stellar mass (Table 3.1). It is immediately apparent that $\operatorname{SFR}(\mathrm{H} \alpha)$ is not directly correlated with the optical depth of extended emission. The galaxy with the highest SFR, NGC 7126, is one that most clearly does not have extended optically thin emission. Furthermore, the galaxy with the second highest SFR, NGC 1482, is optically thick.

A more meaningful comparison is to look at the $\Sigma_{\mathrm{SFR}}$ and the specific SFR. These measures of the SFR take into account the sizes and masses of the galaxies. A more massive galaxy would need a higher SFR to produce enough feedback for ionizing radiation to escape. Table 3.3 shows that NGC 178 and NGC 7126, which show no evidence for extended emission, do have low $\Sigma_{\mathrm{SFR}}$, which is consistent with our expec- 
Table 3.3. Star Formation Rates

\begin{tabular}{|c|c|c|c|c|c|c|c|}
\hline Galaxy $^{a}$ & $\begin{array}{c}\log \left(\mathrm{M}_{\mathrm{HI}}\right) \\
\mathcal{M}_{\odot}\end{array}$ & $\begin{array}{c}\mathrm{SFR} \\
{\left[\mathcal{M}_{\odot} / \mathrm{yr}\right]}\end{array}$ & $\begin{array}{l}\Sigma_{\mathrm{SFR}}{ }^{a} \\
{\left[\frac{\mathcal{M}_{\odot}}{y_{r k p c^{2}}}\right]}\end{array}$ & $\begin{array}{l}\text { sSFR } \\
\mathrm{Gyr}^{-1}\end{array}$ & $\begin{array}{c}\text { Ref }^{b} \\
\text { Bubbles? }\end{array}$ & Wind or & $\mathrm{M}_{\mathrm{HI}} / \mathrm{M}_{*}^{c}$ \\
\hline \multicolumn{8}{|c|}{ Optically thin extended emission } \\
\hline NGC 3125 & $\cdots$ & 0.461 & 0.0826 & 0.374 & $(1), \mathrm{n} / \mathrm{a}, 2$ & $\mathrm{Y}(3)$ & $\cdots$ \\
\hline NGC 5253 & 8.22 & 0.428 & 1.04 & 0.382 & $(1),(4),(5)$ & $Y(3)$ & 0.006 \\
\hline \multicolumn{8}{|c|}{ Optically thick extended emission } \\
\hline NGC 1482 & 9.13 & 3.39 & 0.0271 & 0.093 & $(1),(6),(7)$ & $\mathrm{Y}(8)$ & 0.037 \\
\hline NGC 1705 & 7.96 & 0.057 & 0.0091 & 0.241 & $(1)),(4),(9,7)$ & $\mathrm{Y}(10)$ & 0.389 \\
\hline He 2-10 & 8.49 & 0.872 & 0.340 & 0.276 & $(1),(11),(12)$ & $\mathrm{Y}(13)$ & 0.098 \\
\hline \multicolumn{8}{|c|}{ No detected extended emission } \\
\hline NGC 178 & 9.40 & 0.549 & 0.019 & 0.315 & $(14),(4),(4)$ & $\mathrm{N}$ & 1.445 \\
\hline NGC 7126 & $10.57^{d}$ & 5.21 & 0.0467 & 0.188 & $(14),(4),(4)$ & $\mathrm{N}$ & $1.349)$ \\
\hline
\end{tabular}

Note: ${ }^{a} \Sigma_{\mathrm{SFR}}$ is calculated from the $\mathrm{H} \alpha$ SFR using either the radius of the ionized emission (Calzetti et al., 2010) or the effective $\mathrm{H} \alpha$ radius (Oey et al., 2007).

${ }^{b}$ References: the first reference listed in the column is for the $\mathrm{H} \alpha \mathrm{SFR}$, the second is for $\mathrm{M}_{\mathrm{HI}}$, and the third is for $R_{\mathrm{H} \alpha}$ (1) Calzetti et al. (2010); (2) Gil de Paz et al. (2003);

(3) Marlowe et al. (1995); (4) Meurer et al. (2006); (5) Calzetti et al. (1999);

(6) Hota \& Saikia (2005); (7) Kennicutt et al. (2009); (8) Veilleux \& Rupke (2002);

(9) Kennicutt et al. (2008); (10) Meurer et al. (1992); (11) Sauvage et al. (1997);

(12) Johnson et al. (2000); (13) Méndez et al. (1999); (17) Oey et al. (2007)

${ }^{c} M_{*}$ from Table 3.1 .

${ }^{d} \log \left(M_{\mathrm{HI}}\right)$ is a combination of NGC 7126 and NGC 7125. 
tations. However, distinguishing between the galaxies that have optically thin and thick emission is more challenging. NGC 5253 has the highest $\Sigma_{\text {SFR }}$, but NGG 3125 has $\Sigma_{\text {SFR }}$ lower than He 2-10, which is likely optically thick. One explanation is that the higher SFR in He 2-10 contains contamination from its AGN. However, the AGN in He 2-10 is not particularly luminous in $\mathrm{H} \alpha$ (Reines et al., 2011) making the significance of its contribution unclear. NGC 1705 has comparatively low $\Sigma_{\text {SFR }}$. Paired with the lower $\Sigma_{\text {SFR }}$ of NGC 1482, the rates are consistent with the hypothesis that optical depth is correlated with $\Sigma_{\mathrm{SFR}}$. However, we cannot extrapolate any conclusions on trends with $\Sigma_{\mathrm{SFR}}$ based only on our findings for these individual galaxies.

The comparison between sSFR is similarly fraught. NGC 5253 and NGC 3125 do have among the highest sSFR, but their sSFRs are not significantly higher than those of the galaxies with optically thick emission. Furthermore, NGC 178, which doesn't have clear evidence for extended emission, has a rather high sSFR. Thus, our small sample supports previous work in its conclusion that strong SFR is necessary but not sufficient for significant $f_{\text {esc }}$ (Heckman et al., 2001). We caution the reader that our sample is small, and the range of star-formation rates is also small. Therefore, our sample does not provide the leverages to firmly establish any apparent correlation between optical depth and $\Sigma_{\mathrm{SFR}}$ or sSFR. As an additional consequence of the small sample size, the galaxies that seem anomalous, such as NGC 178 with its high sSFR but lack of extended emission and He 2-10, which is optically thick despite its high $\Sigma_{\mathrm{SFR}}$, may not be significant outliers.

In addition to SFR, the recent star formation history will have an influential role on the escape of ionizing radiation. As a stellar population ages, the rate of ionizing photons produced $\left(Q_{0}\right)$ decreases and the hardness of said radiation field softens. The ages of the clusters responsible for the ionization cones in both NGC 5253 and NGC 3125 are between 2-5 Myr old (Calzetti et al., 1997; Westera et al., 2004; Chandar et al., 2005; Raimann et al., 2000). In contrast with this, NGC 1705 is in a post-burst state (cluster ages $\sim 10-15 \mathrm{Myr}$ ) in which most of the $\mathrm{O}$ stars in the main super star clusters have died off (Heckman \& Leitherer, 1997). Thus, the softer and fainter B stars dominate the radiation field and do not provide enough ionizing 
radiation to allow for escape. This may similarly offer a partial explanation for the line ratios observed in He 2-10. While the main star forming region, embedded in the center of the galaxy, contains many young clusters with ages ranging from 2-20 Myr (Johnson et al., 2000), the second star-forming region, further towards the eastern edge of the galaxy is likely much older with fewer of its massive stars remaining. Furthermore, visual examination of the images of He 2-10 show closed bubbles, rather than fractured filaments (Figure 3.4). We note that for radiation fields dominated by late type stars, the relationship between the ionization parameter map and the optical depth is complicated. In these situations, a transition to [SII] dominated gas does not necessarily indicate an optically thick nebula (Pellegrini et al., 2012). If the radiation field is soft but sufficiently strong, it is possible that not enough gas is present to absorb the lower energy photons, yet, the ionization parameter map still exhibits a transition zone to [SII].

Furthermore, whether the population is a single burst or an extended burst also influences the fate of ionizing radiation. While feedback generates low density passageways in the ISM through which ionizing radiation can escape, simulations of superbubble evolution show that there is a delay between superbubble formation and the actual escape of ionizing radiation (Dove et al., 2000; Fujita et al., 2003). During the phase prior to superbubble break-out, ionizing radiation gets trapped by the bubble walls (Fujita et al., 2003). Once the bubble breaks out of the disk, ionizing radiation may escape relatively unhindered. However, by this point in time, the rate of ionizing photons produced by the massive star population is considerably weaker than in the first few Myr (Dove et al., 2000; Fujita et al., 2003). If the starburst occurs over a short duration, essentially a single burst, then it is less likely to leak significant ionizing radiation. If instead the burst is more extended, the later episodes of star formation can capitalize on the passageways carved by prior star forming episodes.

Our data support this scenario. In NGC 5253, the current burst of star formation occurred within the last 3-5 Myr (Calzetti et al., 1997). While it is unlikely that any superbubbles formed from this epoch will have broken from the disk, there is evidence for prior star formation episodes with ages on the order of 10-100 Myr (Caldwell \& 
Phillips, 1989). Thus, the ionization cone in NGC 5253 probably formed through low density regions cleared out by feedback from earlier episodes of star formation. In NGC 3125, the super star cluster at the base of the ionization cone has an age between 1-3 Myr, while the region surrounding it has an overall mean age of between 8-10 Myr (Westera et al., 2004; Chandar et al., 2005). While the initial episode of star formation in this burst is younger than that shown for NGC 5253, the dominant star formation is on the periphery of the galaxy, which makes it easier for ionizing radiation to escape (Gnedin et al., 2008). Furthermore, Raimann et al. (2000) stacked a sample of similar HII galaxies in which NGC 3125 was the dominant source and found that $65 \%$ of the stellar population is younger than $5 \mathrm{Myr}, 32 \%$ is between 5 and 100 Myr, and the remaining 3\% of the population us is older than 100 Myr. While the SFH analysis is not based solely on NGC 3125 it does point in the direction of NGC 3125 having multiple recent episodes of star formation.

The young ages of the ionizing populations in NGC 3125 and NGC 5253 may represent a special age range needed to find escaping ionizing radiation. In a recent paper, Jaskot \& Oey (2013) study a sample of $z \sim 0.1-0.3$ galaxies whose extremely high $[\mathrm{OIII}] /[\mathrm{OII}]$ suggest that they are likely optically thin. These galaxies all have ionizing populations that are between 3-5 Myr (Jaskot \& Oey, 2013), similar to the ages observed in galaxies with ionization cones studied in this work. Furthermore, the two local galaxies that have direct $f_{\text {esc }}$ measurements, both have significant populations of young stars (Leitet et al., 2013, 2011).

Finally, it has been suggested that the mass of the galaxy is correlated with the escape fraction of ionizing radiation. There are some simulations that predict higher $f_{\text {esc }}$ with larger galaxy mass (e.g., Gnedin et al., 2008). Others claim the opposite because the smaller galaxy's weaker potential well would make escape easier (Yajima et al., 2011; Razoumov \& Sommer-Larsen, 2010). Based on our small sample here, we do not find evidence for or against either claim. The two galaxies for which the extended ionized gas is optically thin have masses that are in the middle to low range of masses in the sample (Tables 3.1 and 3.3). Interesting to note, these two galaxies have significantly higher fractions of HI relative to the stellar mass of the galaxy (Table 
3.3) than the sample galaxies with extended ionized gas. Meanwhile, NGC 5253 has an exceedingly low HI mass fraction, even when compared to the other galaxies with winds. This is consistent with the idea that the clearing, or consumption, of the neutral gas in the galaxy plays a role in regulating the escape of ionizing radiation.

\subsection{Conclusions}

The passage of ionizing radiation through, and possibly out of, a galaxy has ramifications on our understanding of cosmic reionization. In this paper, we study the extended, low surface brightness, emission-line gas of seven dwarf starburst galaxies. Using narrowband [SIII], [SII], and $\mathrm{H}_{\alpha}$ images and the technique of ionization parameter mapping, we evaluate the optical depth of the extended emission.

In two of the galaxies, we discover optically thin ionization cones extending along the minor axis. These cones suggest that ionizing radiation is escaping the main body of the galaxy. The narrow morphology suggests that ISM morphology is a critical determinant of whether ionizing photons will escape a galaxy. In three of the galaxies, we find that the established galactic winds are most likely optically thick. These galaxies, despite the presence of strong feedback do not show clear evidence for escaping ionizing radiation. The two remaining galaxies, despite the strong star formation show little evidence of extended emission.

Our most convincing examples of optically thin emission are found in narrow ionization cones. These narrow cones illustrate a scenario in which the covering fraction of Lyman continuum is significantly less than unity, as has been suggested by other work (e.g., Nestor et al., 2011). Furthermore, both cones are aligned with the minor axis of the galaxy, indicating a preferred direction. The small opening angle and preferred direction suggest that unless the galaxy orientation is such that the axis of escape aligns with the line of sight, it will be challenging to directly detect escaping radiation. If other starbursts are similar to NGC 3125 and NGC 5253, an orientation bias at least partially explains the low detection rate of ionizing radiation in starburst galaxies and LBGs.

In addition to observational biases, we explore the galactic properties that may 
regulate the escape of ionizing radiation. While galactic winds and bubble activity encourage escaping radiation, not all starbursts with galactic wind have escaping Lyman continuum. One explanation is that by the time the bubble or wind breaks out of the ISM, the ionizing population has aged and is no longer producing ionizing radiation as prodigiously (e.g., Dove et al., 2000; Fujita et al., 2003). Alternatively, the ionizing population is too young to have carved sufficient low-density paths out of the ISM for escape. This suggests an optimal age between 3-5 Myr for the escape of ionizing radiation. Based on the recent star-formation histories of NGC 3125 and NGC 5253, a starburst with a few recent star forming episodes strikes the balance between the time it takes for bubbles to burst and maximizing the number of ionizing photons produced that have clear passage out of the galaxy.

Another potential contributing factor is the concentration of star formation in the burst. In the examples of optically thin ionization cones, we see that they emanate from specific, concentrated star forming regions, rather than extended star-forming areas. In contrast, NGC 7126 shows no sign for feedback-driven extended emission, and has its copious star formation spread throughout the disk of the galaxy. This suggests that concentrated star formation will be more effective at clearing out the ISM. However, we must be careful in drawing strong conclusions from so few objects. He 2-10 and NGG 1705, which also have concentrated star formation, are most likely optically thick to ionizing radiation. Thus, while the concentration of star formation is important, the interplay between the star formation and the ISM morphology most likely regulates the escape of ionizing radiation from starbursts and Lyman break galaxies. 

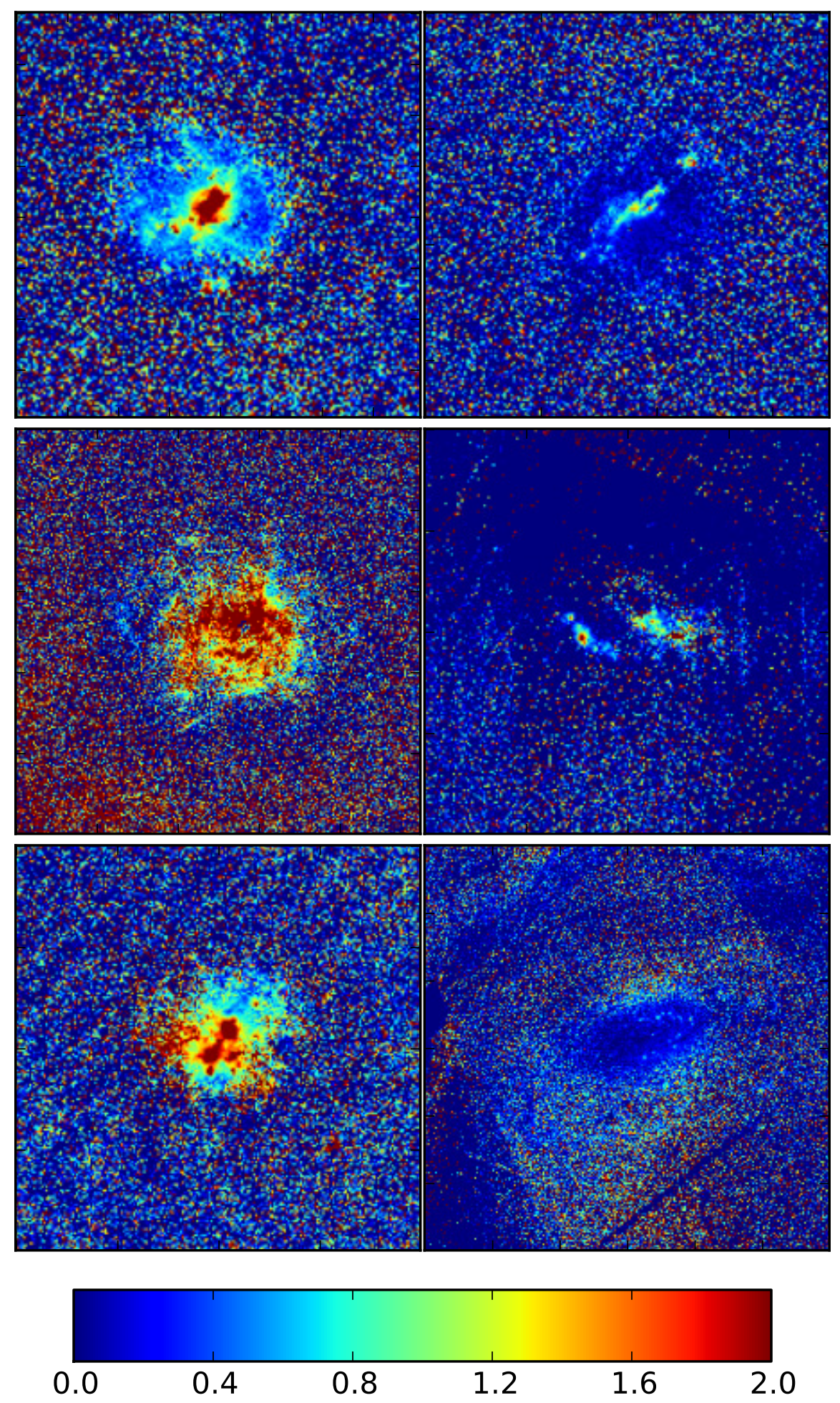

Figure 3.9. Emission-line ratio maps of [SIII]/[SII] for the galaxies in our sample. The narrowband images were binned $3 \times 3$ before generating the ratio map, resulting in $0.6^{\prime \prime}$ pixel sizes. The scale from blue to red corresponds to a line ratios from 0 to 2. The orientation of the figures has North up and East to the left. 

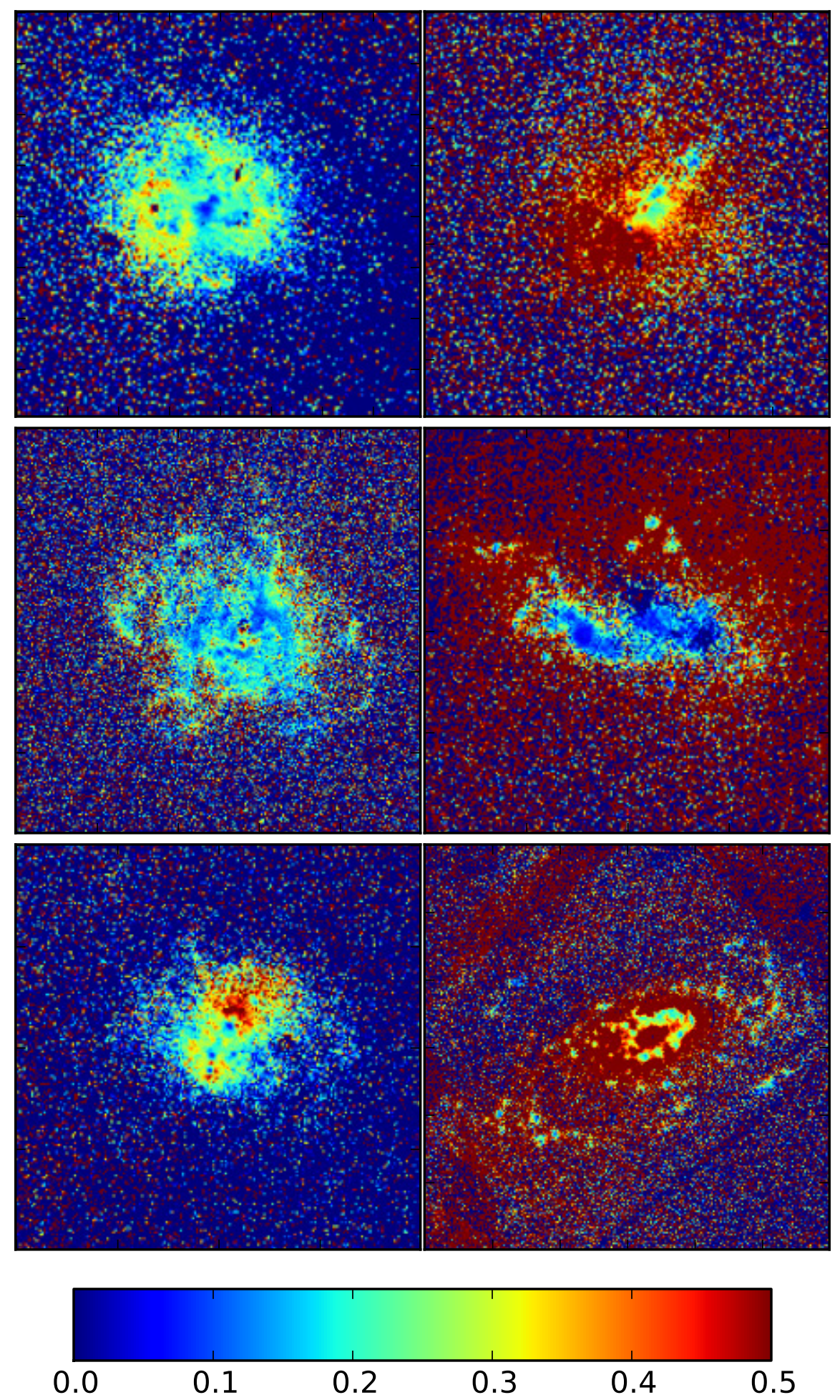

Figure 3.10. Emission-line ratio maps of $[\mathrm{SII}] / \mathrm{H} \alpha$ for the galaxies in our sample. The narrowband images were binned $3 \times 3$ before generating the ratio map, resulting in $0.6^{\prime \prime}$ pixel sizes. The scale from blue to red corresponds to a line ratios from 0 to 0.5 . The orientation of the figures has North up and East to the left. 

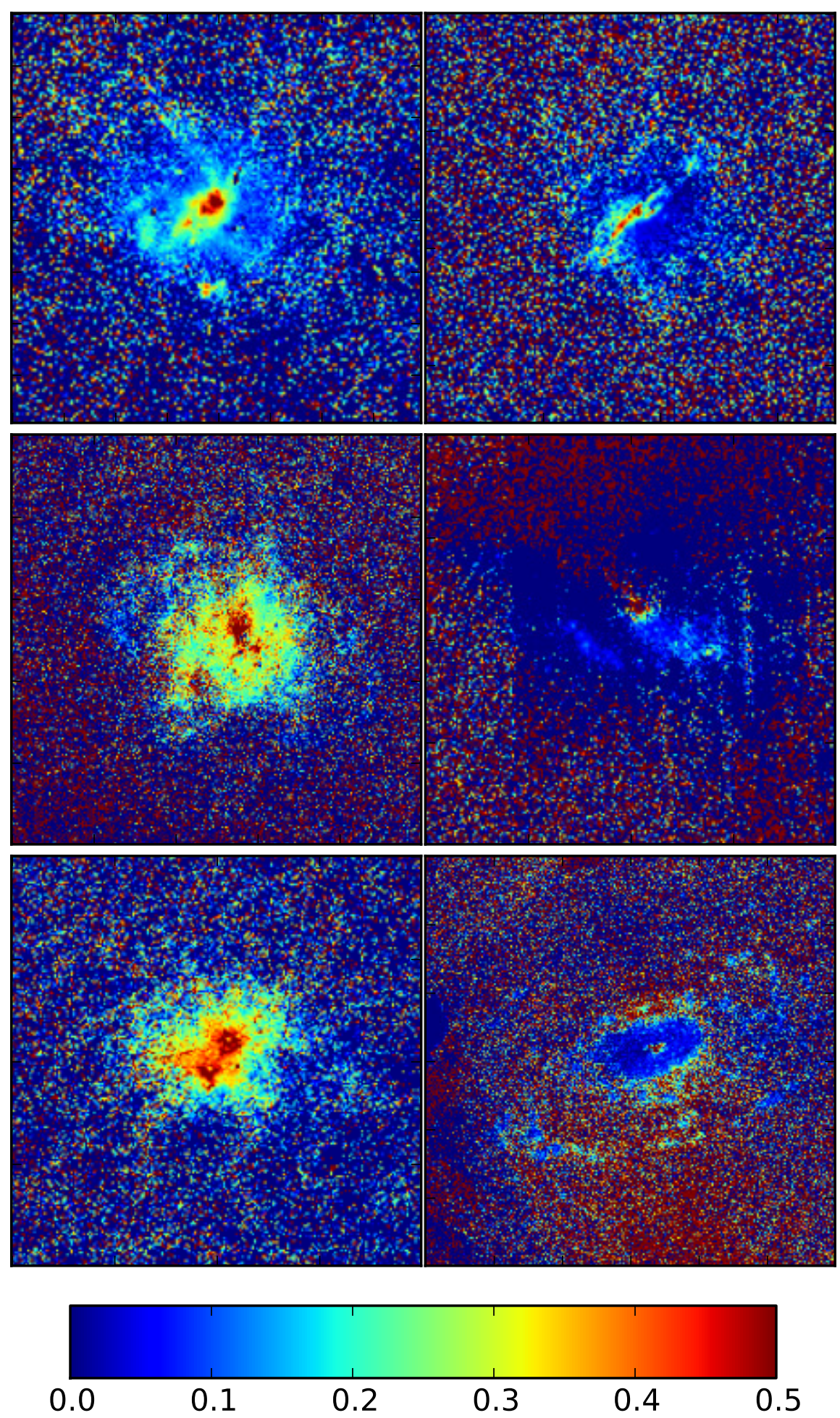

Figure 3.11. Emission-line ratio maps of $[\mathrm{SIII}] / \mathrm{H} \alpha$ for the galaxies in our sample. The narrowband images were binned $3 \times 3$ before generating the ratio map, resulting in $0.6^{\prime \prime}$ pixel sizes. The scale from blue to red corresponds to a line ratios from 0 to 0.5 . The orientation of the figures has North up and East to the left. 


\section{CHAPTER 4}

\section{Single-Star HII Regions as a Probe of Massive Star SEDs}

\subsection{Introduction}

Through the effects of radiative, mechanical and chemical feedback, massive stars play a critical role in shaping the galaxies in which they reside. These stars ionize their local interstellar medium (ISM) and form bright HiI regions that can be observed in distant galaxies, even where the individual stars are not resolved. Since the HII region emission-line spectrum is sensitive to both the spectral energy distribution (SED) of the ionizing source and the properties of the nearby gas, it is a diagnostic for galaxy properties, such as star formation rates and histories (e.g., Leitherer et al., 1999; Hunter \& Massey, 1990; Kennicutt et al., 2000), properties of the ionizing stellar population and the slope of the initial mass function (e.g., Baldwin et al., 1981; Kaler, 1978; Rigby \& Rieke, 2004; Stasińska \& Leitherer, 1996; Copetti et al., 1986; Dufour, 1975), and the chemical abundances and chemical evolution of galaxies (e.g., Kewley \& Dopita, 2002; Edmunds \& Pagel, 1984; Bresolin et al., 1999).

The shape of the stellar SED is particularly important for these diagnostics. For example, it determines the rate of ionizing photons $\left(Q_{0}\right)$ emitted by massive stars, upon which the commonly used $\mathrm{H} \alpha$ star formation rate indicator depends (Kennicutt, 1983). Other popular diagnostics use flux ratios of lines with different ionization potential as diagnostics for the effective temperature $\left(T_{\text {eff }}\right)$ of the ionizing stars (e.g., Vilchez \& Pagel, 1988; Stoy, 1933; Zanstra, 1927; Dors \& Copetti, 2003). Thus, the

shapes of massive star SEDs play key roles in deriving the physical properties of nebulae and stars from observed HII region spectra. 
Since massive stars radiate most of their flux at FUV wavelengths that are relatively inaccessible to observations, we are dependent on the predictions generated by stellar atmosphere models to describe the properties and SEDs of massive stars. To reproduce the stellar SED, the models need to take into account the effects of non-LTE conditions, stellar winds, and line-blanketing (e.g., Kudritzki \& Hummer, 1990; Schaerer \& Schmutz, 1994; Stasińska \& Schaerer, 1997; Lanz \& Hubeny, 2003; Pauldrach et al., 2001). Calculating these in detail is both challenging and computationally time-consuming. Therefore, the model atmospheres currently available incorporate these processes with different approximations, by balancing an exact treatment of the physics against faster computation. The different treatments affect the shape of the SED and the properties derived from them (e.g., Simón-Díaz \& Stasińska, 2008; Voges et al., 2008; Giveon et al., 2002). It is therefore crucial to know how well these different atmosphere models represent the true SED of these stars.

Since the HII region emission-line spectra strongly depend on the shape of the ionizing SED, a direct comparison between the emission lines from observed HII regions and those predicted by photoionization simulations will reveal how well the different atmosphere models represent the SEDs of massive stars. Earlier such work by Stasińska \& Schaerer (1997) found that line blanketed models show significant improvement over previous un-blanketed models. Observational studies of both OB (e.g., Oey et al., 2000; Bresolin et al., 1999) and Wolf-Rayet nebulae (Esteban et al., 1993; Crowther et al., 1999) support the importance of the treatment of line blanketing on the resultant HII region spectrum. Comparison of HII region infrared line ratios to simulations confirmed that line blanketing is a crucial process to include in the models and showed that the SEDs produced by plane-parallel atmospheres are too soft (e.g., Martín-Hernández et al., 2002; Morisset et al., 2002; Giveon et al., 2002).

However, HII regions are complex systems with clumpy and irregular gas distributions. Furthermore, most studies use nebulae that contain many stars that are distributed throughout the ionized region. Properly accounting for these multiple 
ionizing sources and complex gas distributions is a major challenge to this approach and vastly limits the constraints that one can put on the SEDs (e.g., Ercolano et al., 2007). Our study circumvents this challenge by using single-star HII regions in the nearby Large Magellanic Cloud (LMC). The HII regions are spatially resolved and spherical, which makes the modeling more straightforward. We use photoionization simulations to evaluate how well atmosphere models represent the shape of the massive star SED.

\subsubsection{Description of the Model Atmosphere Codes}

Our goal in this work is to understand how well the OB atmosphere-model grids that are available to general users represent ionizing stars. For this reason, we use publicly available grids, rather than fitting each stellar spectrum in detail to obtain the appropriate model. We use the O-star grid presented in Smith et al. (2002), hereafter SNC02, in addition to the CoStar (Schaerer \& de Koter, 1997), TLUSTY (Lanz \& Hubeny, 2003), and WM-basic (Pauldrach et al., 2001) grids that are already available in the stellar atmosphere library of CLOUDY (Ferland et al., 1998). These massive-star atmosphere codes include important, but complex, physical processes such as non-LTE conditions and the effects of metal lines and winds on the transmitted spectrum. Including these processes in detail is computationally expensive. Therefore, research groups use various methods and algorithms to approximate some of these processes. The differences in the algorithms used result in non-negligible differences in the SEDs produced by different atmosphere codes (e.g., Simón-Díaz \& Stasińska, 2008).

All the atmosphere codes considered in this work solve for non-LTE radiation transfer. Typically this is accomplished by grouping lines of similar excitation energies together and applying the same non-LTE correction to the populations in that group (e.g., Hubeny \& Lanz, 1995; Pauldrach et al., 2001). In some atmosphere codes, such as CoStar and WM-basic, the non-LTE solution includes the effects of spherically expanding winds, while others, such as TLUSTY, assume a plane-parallel geometry.

Metal lines in the UV impact the emergent SED in two ways, line blocking and 
line blanketing. Line blocking refers to the absorption and scattering of the emergent flux due to the higher opacity in the line. Line blocking will increase the temperature in the deeper layers of the star because of the scattering of radiation back towards the star, an effect known as backwarming (Pauldrach et al., 2001; Martins et al., 2005). Line blanketing refers to the redistribution of energy to regions where the metal lines are not so densely packed (Pauldrach et al., 2001). The main method used to include line blocking and blanketing is the opacity sampling method. For this method, the opacities are evaluated for a grid of frequency points. The approximation approaches the exact solution as the code increases the number of frequency points that it samples. Of the three codes considered here, CoStar has the most approximate treatment. CoStar incorporates the opacity sampling using $\sim 50 \AA$-wide bands in the Monte Carlo radiative transfer solution (Schaerer \& Schmutz, 1994). In contrast, the TLUSTY atmosphere code includes 180,000-200,000 frequency points in the opacity sampling grid (Lanz \& Hubeny, 2003). The WM-basic atmosphere code is solved in two parts. First, the radiative transfer is solved with a fast approximate treatment that samples $\sim 1,000$ frequency points in the Lyman continuum. This fast solution is repeated iteratively and generates starting values for the final solution. The final solution consists of fewer iterations that solve the radiative transfer with an exact treatment of the line-blanketing (Pauldrach et al., 2001).

In addition to line-blanketing by metal lines, stellar winds change the shape of the ionizing SED at high energies (e.g., Sellmaier et al., 1996). This is particularly important near the $54.4 \mathrm{eV}$ ionization potential of HeII (Gabler et al., 1989). Both CoStar and WM-basic calculate the non-LTE solution for expanding atmospheres. In the WM-basic atmosphere code, the standard opacity sampling is modified to take into account line shifts due to the expanding winds. These line shifts effectively increase the frequency range that can be blocked by a given line (Pauldrach et al., 2001).

While SNC02 is generated with the WM-basic code, they use a different set of assumptions for the stellar parameters in calculating the SEDs. Specifically, they determine the appropriate mass-loss rate and terminal velocities a priori using the 
empirical relations from Prinja et al. (1990), Lamers et al. (1995) and Kudritzki \& Puls (2000). To ensure the final atmospheres have these parameters, they manually adjust the radiative acceleration by changing the force multipliers used in the code (Smith et al., 2002). In contrast, the WM-basic grid included in CLOUDY obtains the mass-loss rate and wind terminal velocity from the solution of the atmosphere code itself (Pauldrach et al., 1998). We note that the SNC02 grid was previously implemented in STARBURST99 (Leitherer et al., 1999).

\subsection{Observations and Method}

\subsubsection{Observations}

To evaluate the atmosphere grids described above, we first construct a sample of single-star Hı regions. We use the narrow-band emission-line images from the Magellanic Clouds Emission Line Survey (MCELS; Smith et al., 2005) to select small, spherical HiI regions that are likely ionized by a single star. Table 4.1 lists the properties of these HII regions. The first five columns give the MCELS (Pellegrini et al., 2012), DEM (Davies et al., 1976) and stellar designations, and the (J2000) positions. The next three columns list observed properties of the ionizing stars. The ninth column has the observed radius of the HiI region in parsecs as measured from the $\mathrm{H} \alpha$ MCELS image. Figure 4.1 shows both $\mathrm{H} \alpha$ and three-color composites in [OIII], [SII], and $\mathrm{H} \alpha$ of the Hir regions in our sample.

We used the Inamori-Magellan Areal Camera \& Spectrograph (IMACS) on the Magellan Baade Telescope at Las Campanas Observatory to obtain both long slit spectra and Bessell $B$ and $V$ images. Our data were collected on the nights of 2008 January 29-31 using the f/4 configuration. In this setup, IMACS has an eight-chip mosaic CCD that has a total of $8,000 \times 8,00015 \mu \mathrm{m}$ pixels, each of which corresponds to $0 . " 11$. The seeing was good over the observing run, resulting in a final spatial resolution of $\sim 1.6^{\prime \prime}$.

The long slit observations consist of $3 \times 1200$ s exposures using a $0 .{ }^{\prime \prime} 7$ slit. We

use the $600 \mathrm{l} \mathrm{mm}^{-1}$ grating, which has a spectral resolution $R \sim 2730$ at $\mathrm{H} \alpha$. The 


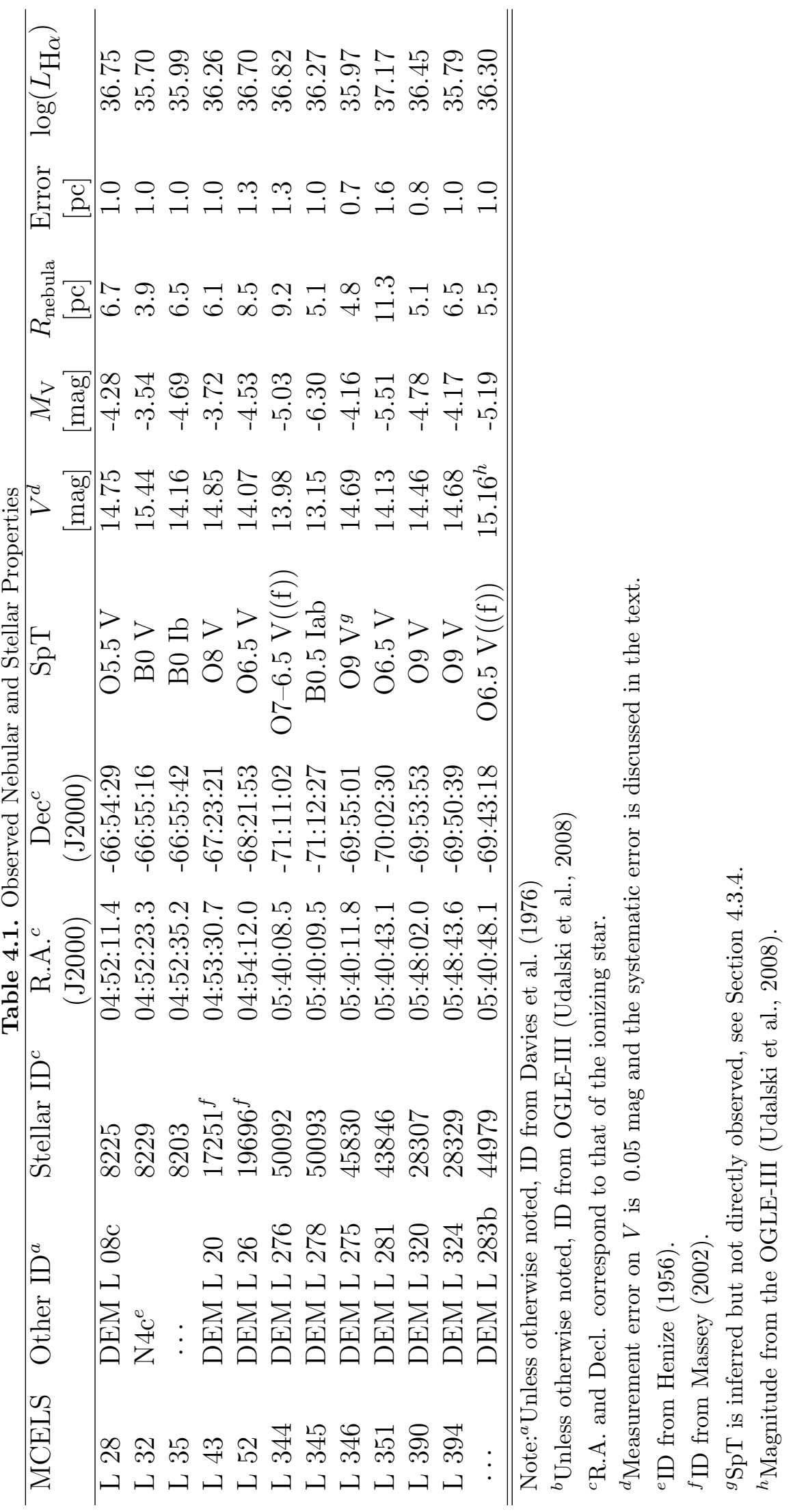


data are binned by 2 and 4 in the dispersion and spatial directions, respectively, which results in $\Delta \sim 0.76 \AA$ per pixel. For the first night of the observing run the spectral coverage is $3700-5900 \AA$, while for the nights of 2008 January 30 and 31 the wavelength coverage is $3700-6740 \AA$ due to a different grating tilt.

We use standard IRAF ${ }^{1}$ procedures for the data reduction. The spectra are extracted with the IRAF task apextract using separate apertures for the nebula and star. The extraction includes a local background subtraction. We extract the nebular spectra in two or more apertures, which are selected to exclude any stars along the slit. The apertures otherwise span the entire nebula. We sum those spectra to obtain the total emission from the nebula along the slit. Figures 4.2 and 4.3 show the stellar and nebular spectra for the objects in our sample. The intensities are scaled to an arbitrary value for presentation, and the gaps between the chips are assigned a value equal to the continuum level. We flux calibrate the spectra using standard stars LTT 3218, LTT 1788, LTT 2415, LTT 2754 and EG 21 (Hamuy et al., 1994).

We measure the emission line fluxes from the nebular spectra (Figure 4.3) with the IRAF task splot assuming Gaussian line profiles. We use the reddening equation:

$$
\frac{I(\lambda)}{I(\mathrm{H} \beta)}=\frac{I_{0}(\lambda)}{I_{0}(\mathrm{H} \beta)} 10^{-c(f(\lambda)-f(\mathrm{H} \beta))}
$$

to find the reddening coefficient, $c(\mathrm{H} \beta)$. Here, $I(\lambda) / I(\mathrm{H} \beta)$ and $I_{0}(\lambda) / I_{0}(\mathrm{H} \beta)$ are the observed and intrinsic Balmer ratios, respectively, and we use the reddening law of Cardelli et al. (1989) to determine $f_{\lambda}$ and $f_{\mathrm{H} \beta}$. For the reddening solution we set the ratio of total to selective extinction, $R_{V}=3.45$, which is appropriate for the LMC (Gordon et al., 2003). The de-reddened line strengths, relative to $\mathrm{H} \beta$, and $c(\mathrm{H} \beta)$ values for each nebula are listed Table 4.2. In Table 4.2, we also include the $\mathrm{H} \beta$ flux that we measure in the slit. Note that the slit width is $0 .^{\prime \prime} 7$ relative to the $0 .^{\prime} 5-2^{\prime}$ sizes of the nebulae. Therefore, the $\mathrm{H} \beta$ flux in Table 4.2 is not representative of the $\mathrm{H} \beta$ flux in the nebula. We calculate the emission-line measurement error as

\footnotetext{
${ }^{1} \mathrm{IRAF}$ is distributed by NOAO, which is operated by AURA, Inc., under cooperative agreement with the National Science Foundation.
} 
$\sqrt{\sigma_{\text {cont }}^{2} N+\sigma_{\text {cont }}^{2} \mathrm{EW} / \Delta}$ (Gonzalez-Delgado et al., 1994). Here, $\sigma_{\text {cont }}$ is the rms in the continuum near the line, $N$ is width of the measured line in pixels, EW is the equivalent width of the line and $\Delta$ is the dispersion of the spectra in $\AA$ per pixel (Gonzalez-Delgado et al., 1994). The reddening error and the flux calibration error together contribute $<10 \%$ error for all our sample, and $\sim 5 \%$ for most of our sample. These sources of error are combined in quadrature to obtain the flux error listed in Table 4.2. 


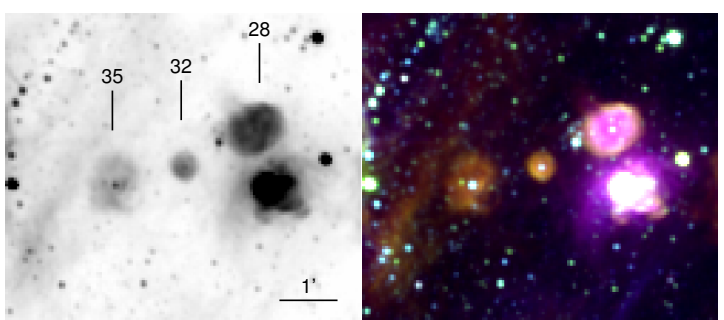

(a) MCELS L 28, MCELS L 32, MCELS L 35

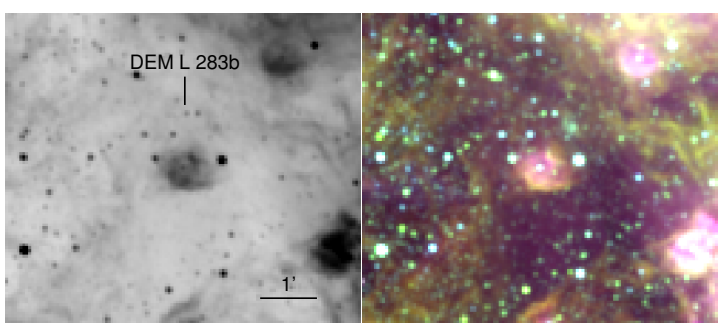

(c) DEM L 283b

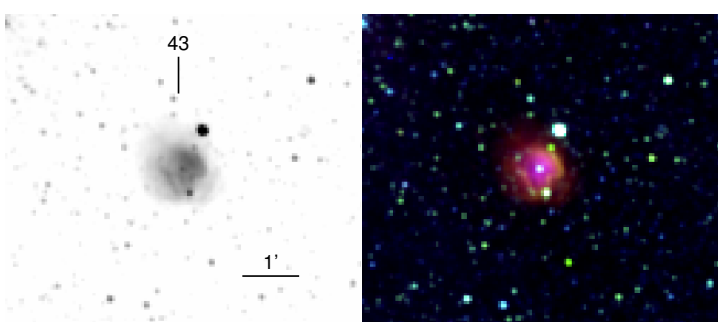

(e) MCELS L 43

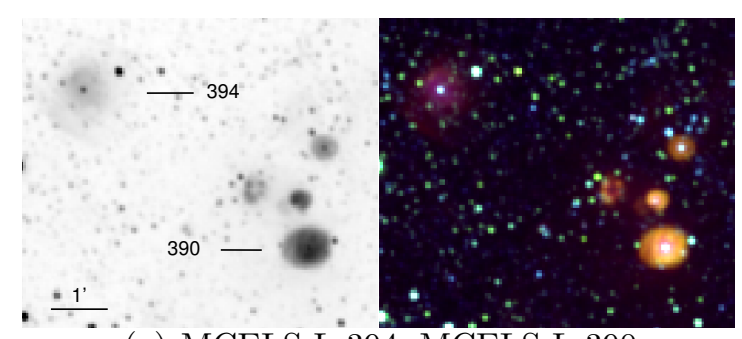

(g) MCELS L 394, MCELS L 390

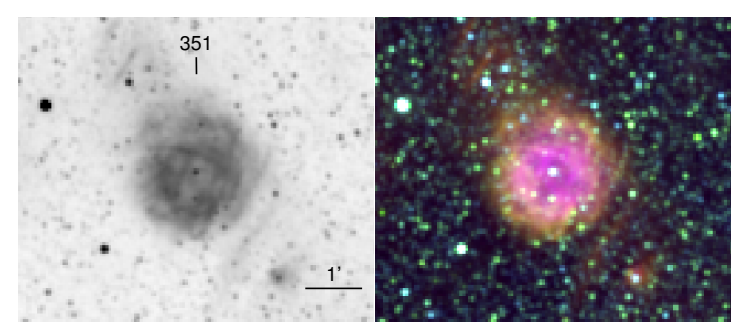

(b) MCELS L 351

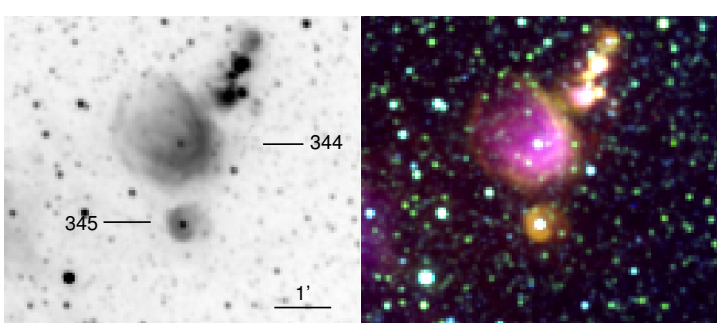

(d) MCELS L 344, MCELS L 345

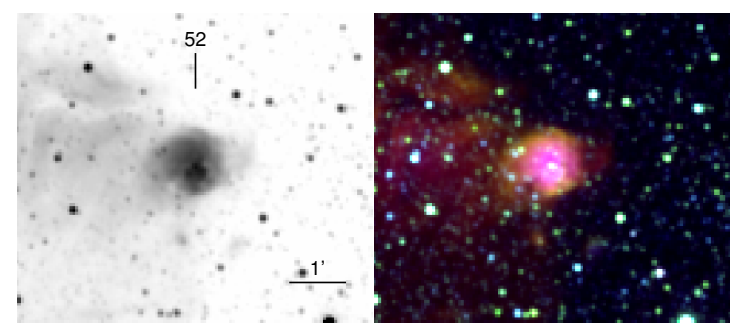

(f) M̈CELS L 52

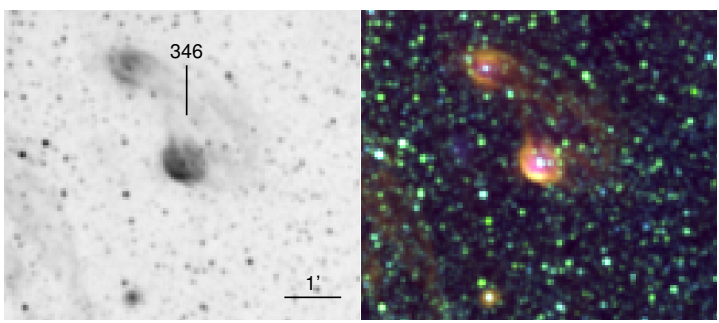

(h) MCELS L 346

Figure 4.1. MCELS images for the objects in our sample. In each image, the left side shows the $\mathrm{H} \alpha$ MCELS image, while the right side is a three-color composite of the MCELS bands; Red, blue, and green are $\mathrm{H} \alpha$, [OIII] $\lambda 5007$, [SII] $\lambda 6720$, respectively. North is up and east is to the left. The subfigures show the following objects: (a)MCELS L 28, MCELS L 32, and MCELS L 35, (b) MCELS L 351, (c) DEM L 283b, (d) MCELS L 344 and MCELS L 345, (e) MCELS L 43, (f) MCELS L 52, (g) MCELS L 390 and MCELS L 394, (h) MCELS L 346 


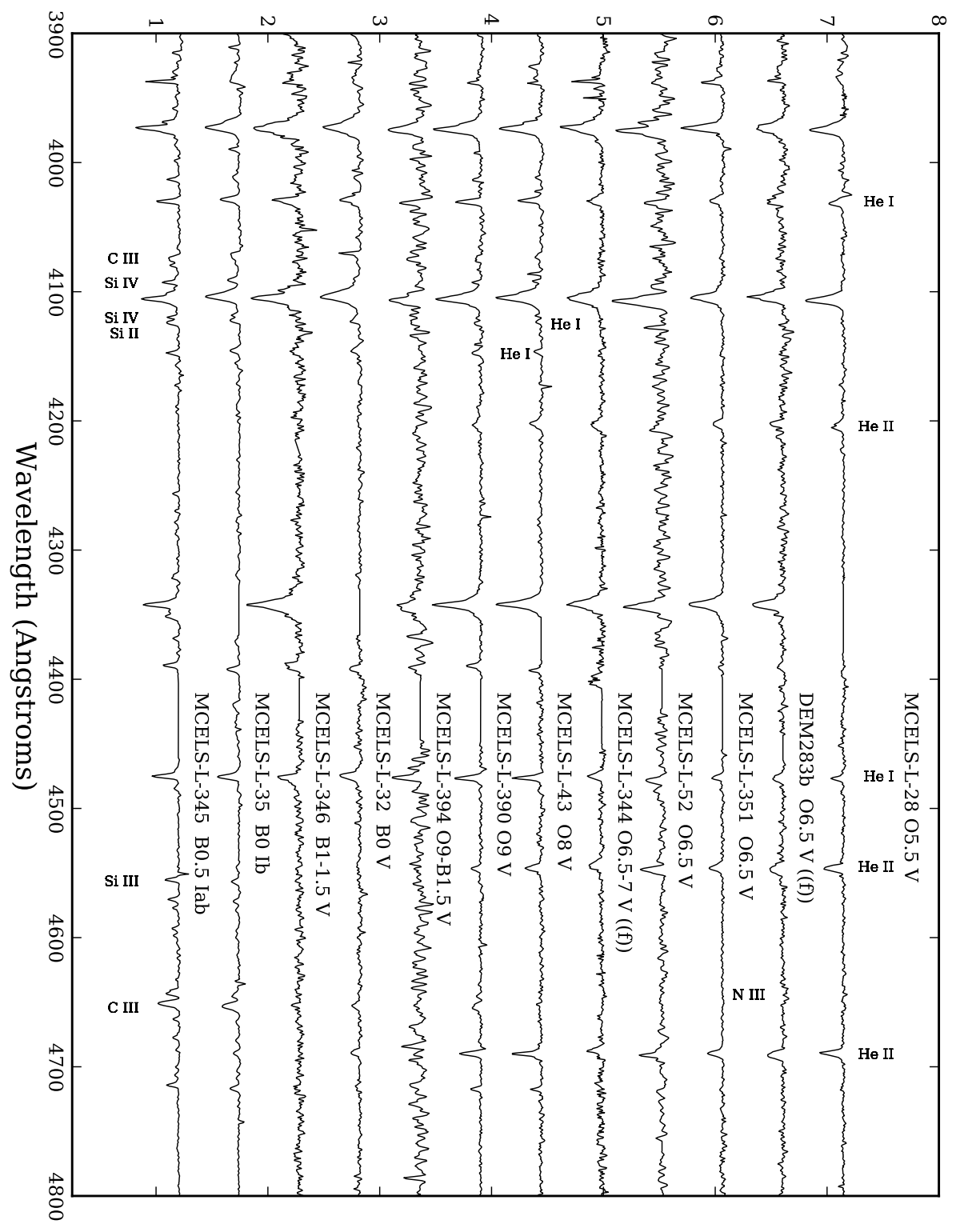

Figure 4.2. Stellar spectra from the ionizing stars of the HiI regions. Spectral type changes toward later type from the top to bottom. The stellar spectra for MCELS L 52 and MCELS L 394 are boxcar smoothed with a smoothing length of 3 pixels. The flat, noiseless regions are the chip gaps, for which we assigned a value of unity. MCELS L 346 is an eclipsing binary, and the stellar spectrum shown here is not the ionizing star, see Section 4.3.4. 


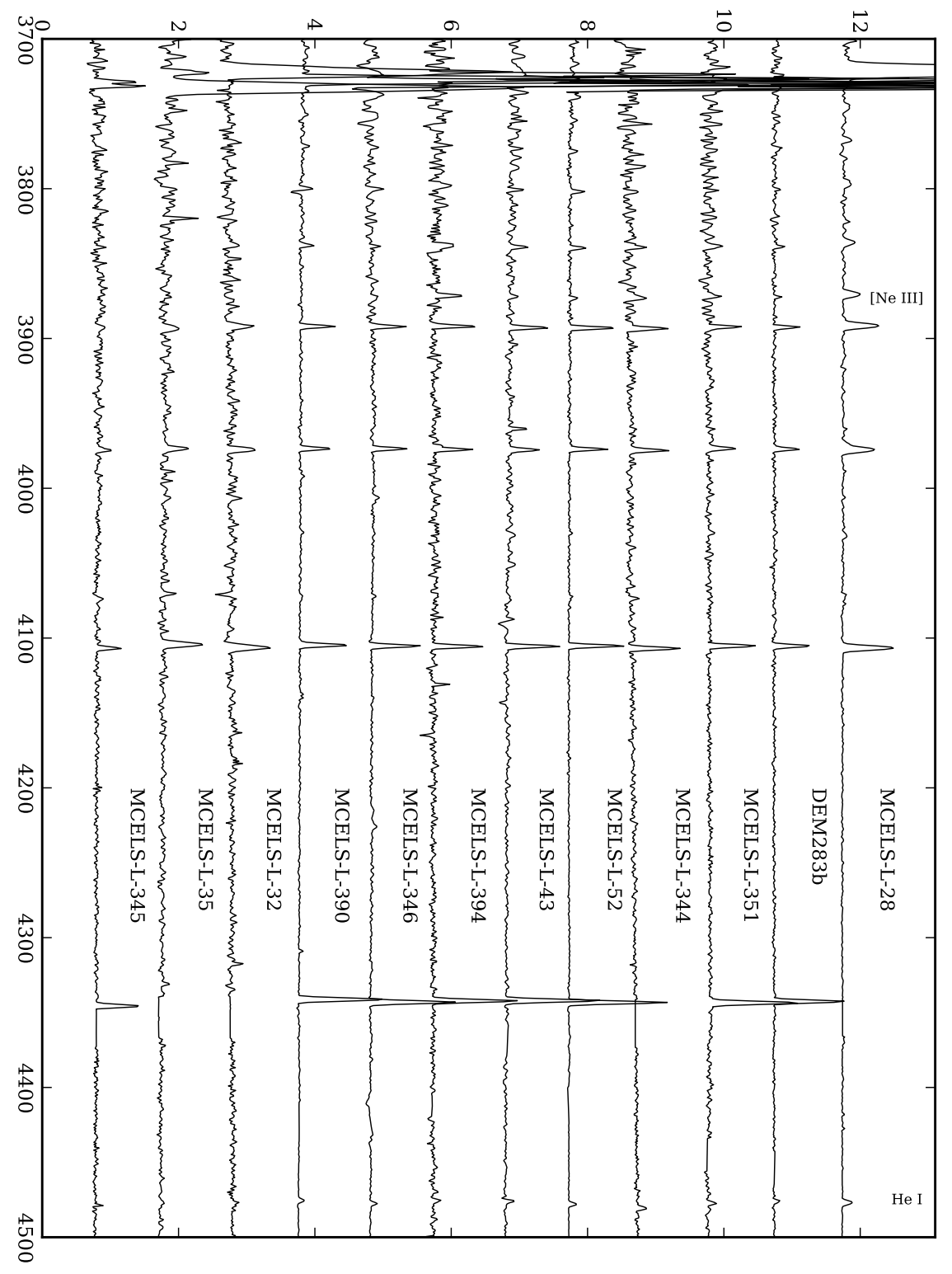

Figure 4.3. Nebular spectra of the HII regions in our sample for 3600-4500 $\AA$. The spectra are scaled to highlight the weaker emission lines. In most of the spectra, the chip gap falls on or slightly red-ward of $\mathrm{H} \gamma$. We assign this region with a value equal to the mean continuum level. 
Table 4.2: De-reddened Emission Lines Fluxes ${ }^{a}$ and Derived Properties

\begin{tabular}{|c|c|c|c|c|c|c|c|c|c|c|c|c|c|}
\hline & $\mathrm{L} 28 \mathrm{n} 1^{b}$ & Error & $\mathrm{L} 28 \mathrm{n} 2^{b}$ & Error & $\mathrm{L} 32 \mathrm{n} 1^{b}$ & Error & $\mathrm{L} 32 \mathrm{n} 2^{b}$ & Error & $\mathrm{L} 35 \mathrm{n} 2^{b, c}$ & Error & $\mathrm{L} 35 \mathrm{n} 2^{b, c}$ & Error & \\
\hline [Оіг] 3726 & 1.503 & 0.115 & $\ldots$ & $\cdots$ & 0.546 & 0.061 & $\cdots$ & $\cdots$ & $\ldots$ & $\cdots$ & $\cdots$ & $\cdots$ & \\
\hline [Оіг] 3726 & 3.690 & 0.390 & 3.446 & 0.081 & 1.464 & 0.211 & 2.283 & 0.168 & 3.705 & 0.416 & 4.407 & $\cdots$ & \\
\hline [Оіг] 3729 & 2.187 & 0.159 & $\cdots$ & $\cdots$ & 0.918 & 0.074 & $\cdots$ & $\cdots$ & $\cdots$ & $\cdots$ & $\cdots$ & $\cdots$ & \\
\hline [NeIII] 3869 & 0.103 & 0.009 & 0.125 & 0.026 & $\cdots$ & $\cdots$ & $\cdots$ & $\ldots$ & $\cdots$ & $\cdots$ & $\ldots$ & $\cdots$ & \\
\hline [OIII] 4363 & 0.009 & 0.002 & $\cdots$ & $\cdots$ & $\cdots$ & $\cdots$ & $\cdots$ & $\cdots$ & $\cdots$ & $\cdots$ & $\cdots$ & $\cdots$ & \\
\hline HeI 4471 & 0.043 & 0.003 & 0.043 & 0.004 & $\ldots$ & $\ldots$ & 0.042 & 0.018 & 0.048 & 0.005 & $\cdots$ & $\ldots$ & \\
\hline [OIII] 5007 & 2.015 & 0.092 & 2.141 & 0.027 & $<0.10$ & 0.009 & 0.049 & 0.009 & 0.068 & 0.005 & 0.069 & 0.008 & \\
\hline HeI 5876 & $\cdots$ & $\cdots$ & 0.118 & 0.002 & $\cdots$ & $\cdots$ & 0.026 & 0.003 & 0.045 & 0.003 & 0.037 & 0.006 & \\
\hline [OI] 6300 & $\cdots$ & $\cdots$ & 0.018 & 0.002 & $\cdots$ & $\cdots$ & $\cdots$ & $\cdots$ & 0.021 & 0.003 & 0.015 & 0.004 & \\
\hline [SIII] 6312 & $\cdots$ & $\cdots$ & 0.014 & 0.001 & $\cdots$ & $\ldots$ & $\cdots$ & $\cdots$ & $\cdots$ & $\ldots$ & $\ldots$ & $\cdots$ & \\
\hline [NII] 6584 & $\cdots$ & $\ldots$ & 0.265 & 0.005 & $\cdots$ & $\cdots$ & 0.360 & 0.014 & 0.495 & 0.017 & 0.482 & 0.044 & \\
\hline$[\mathrm{SII}] 6716$ & $\cdots$ & $\cdots$ & 0.221 & 0.004 & $\cdots$ & $\cdots$ & 0.479 & 0.020 & 0.487 & 0.017 & 0.470 & 0.046 & \\
\hline [SII] 6731 & $\cdots$ & $\ldots$ & $\ldots$ & $\ldots$ & $\ldots$ & $\ldots$ & 0.333 & 0.014 & $\ldots$ & 0.325 & 0.033 & $\cdots$ & \\
\hline $\mathrm{c}(\mathrm{H} \beta)$ & 0.053 & 0.058 & 0.220 & 0.062 & $\cdots$ & $\ldots$ & 0.150 & 0.044 & 0.198 & $\ldots$ & 0.182 & $\ldots$ & \\
\hline $\log (\mathrm{O} / \mathrm{H})$ & -3.51 & 0.10 & $\ldots$ & $\ldots$ & $\cdots$ & $\ldots$ & $\ldots$ & $\ldots$ & $\ldots$ & $\ldots$ & $\ldots$ & $\ldots$ & \\
\hline $\log (\mathrm{N} / \mathrm{O})$ & $\ldots$ & $\ldots$ & -1.34 & $\ldots$ & $\ldots$ & $\ldots$ & -1.20 & $\ldots$ & $\ldots$ & $\ldots$ & $\ldots$ & $\ldots$ & \\
\hline $\log (\mathrm{He} / \mathrm{H})$ & -1.06 & 0.01 & -1.06 & 0.01 & $\cdots$ & $\cdots$ & -1.07 & 0.02 & $\cdots$ & $\cdots$ & $\cdots$ & $\cdots$ & \\
\hline $\mathrm{H} \beta$ flux ${ }^{d}$ & 15.22 & $\ldots$ & 23.57 & $\ldots$ & 2.156 & $\ldots$ & 2.741 & $\ldots$ & 3.196 & $\ldots$ & 3.313 & $\cdots$ & \\
\hline
\end{tabular}

Continued on next page 


\begin{tabular}{|c|c|c|c|c|c|c|c|c|c|c|c|c|c|}
\hline & $\mathrm{L} 35 \mathrm{n} 1^{b, c}$ & Error & $\mathrm{L} 35 \mathrm{n} 1^{b, c}$ & Error & L43 & Error & L52 & Error & $\mathrm{L} 344 \mathrm{n} 1^{b}$ & Error & $\mathrm{L} 344 \mathrm{n} 2^{b}$ & Error & \\
\hline [OІі] 3726 & 1.835 & 0.173 & 1.306 & 0.136 & 1.344 & 0.101 & 1.493 & 0.061 & 1.358 & 0.121 & 1.839 & 0.135 & \\
\hline [OII] 3726 & 3.190 & $\cdots$ & 3.293 & 0.325 & 3.692 & 0.197 & 3.676 & $\cdots$ & 3.299 & $\cdots$ & 3.046 & 0.352 & \\
\hline [Оіг] 3729 & 2.572 & 0.240 & 1.884 & 0.182 & 1.949 & 0.124 & 2.200 & 0.076 & 1.941 & 0.158 & 1.207 & 0.108 & \\
\hline [NeIII] 3869 & $\ldots$ & $\ldots$ & 0.037 & 0.014 & 0.040 & 0.008 & 0.033 & 0.006 & 0.070 & 0.009 & 0.129 & 0.027 & \\
\hline [OIII] 4363 & $\ldots$ & $\ldots$ & $\ldots$ & $\ldots$ & $\ldots$ & $\ldots$ & 0.016 & 0.050 & $\ldots$ & $\cdots$ & $\ldots$ & $\ldots$ & \\
\hline HeI 4471 & 0.008 & 0.003 & 0.011 & 0.004 & 0.040 & 0.005 & 0.028 & 0.003 & 0.042 & 0.004 & 0.044 & 0.008 & \\
\hline [OIII] 5007 & 0.034 & 0.003 & $<0.03$ & $\ldots$ & 1.356 & 0.032 & 1.377 & 0.023 & 1.623 & 0.075 & 1.841 & 0.043 & \\
\hline HeI 5876 & $\ldots$ & $\ldots$ & $\ldots$ & $\ldots$ & 0.115 & 0.005 & 0.110 & 0.030 & $\ldots$ & $\cdots$ & 0.121 & 0.005 & \\
\hline [OI] 6300 & $\ldots$ & $\ldots$ & $\ldots$ & $\ldots$ & 0.034 & 0.008 & 0.014 & 0.002 & $\ldots$ & $\cdots$ & $\ldots$ & $\ldots$ & \\
\hline [SIII]6312 & $\ldots$ & $\ldots$ & $\ldots$ & $\ldots$ & 0.016 & 0.008 & 0.012 & 0.002 & $\ldots$ & $\ldots$ & 0.013 & 0.003 & \\
\hline [NII] 6584 & $\ldots$ & $\ldots$ & $\ldots$ & $\cdots$ & 0.258 & 0.011 & 0.268 & 0.005 & $\ldots$ & $\ldots$ & 0.280 & 0.010 & \\
\hline [SII] 6716 & $\ldots$ & $\ldots$ & $\ldots$ & $\ldots$ & 0.276 & 0.010 & 0.265 & 0.005 & $\ldots$ & $\cdots$ & 0.222 & 0.008 & \\
\hline [SII] 6731 & $\ldots$ & $\ldots$ & $\ldots$ & $\ldots$ & $\ldots$ & $\ldots$ & 0.181 & 0.004 & $\ldots$ & $\cdots$ & 0.151 & 0.006 & \\
\hline $\mathrm{c}(\mathrm{H} \beta)$ & 0.128 & $\cdots$ & 0.133 & $\cdots$ & 0.014 & 0.036 & 0.032 & 0.083 & 0.484 & 0.056 & 0.210 & 0.014 & \\
\hline $\log (\mathrm{O} / \mathrm{H})$ & $\ldots$ & $\ldots$ & $\ldots$ & $\ldots$ & $\ldots$ & $\ldots$ & $>-4.10$ & 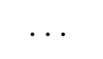 & $\ldots$ & $\ldots$ & $\ldots$ & $\ldots$ & \\
\hline $\log (\mathrm{N} / \mathrm{O})$ & $\ldots$ & $\ldots$ & $\ldots$ & $\ldots$ & -1.34 & $\cdots$ & -1.40 & $\ldots$ & $\ldots$ & $\cdots$ & -1.27 & $\cdots$ & \\
\hline $\log (\mathrm{He} / \mathrm{H})$ & $\ldots$ & $\ldots$ & $\ldots$ & $\ldots$ & -1.09 & 0.01 & -1.10 & 0.01 & $\ldots$ & $\ldots$ & -1.05 & 0.02 & \\
\hline $\mathrm{H} \beta$ flux ${ }^{d}$ & 3.080 & $\ldots$ & 2.384 & $\ldots$ & 4.644 & $\ldots$ & 13.94 & $\ldots$ & 27.81 & $\ldots$ & 15.51 & $\ldots$ & \\
\hline
\end{tabular}




\begin{tabular}{|c|c|c|c|c|c|c|c|c|c|c|c|c|c|c|}
\hline & $\mathrm{L} 345 \mathrm{n} 1^{b}$ & Error & $\mathrm{L} 345 \mathrm{n} 2^{b}$ & Error & L346 & Error & L351 & Error & L390 & Error & L394 & Error & DEM L283b & Error \\
\hline [Оіг] 3726 & 0.592 & 0.046 & 0.592 & 0.172 & 1.543 & 0.134 & 1.251 & 0.179 & 1.520 & 0.066 & 1.667 & 0.287 & 1.570 & 0.174 \\
\hline [OII] 3726 & 1.487 & $\ldots$ & 1.144 & $\cdots$ & 3.779 & 0.201 & 3.271 & 0.546 & 3.919 & 0.452 & 4.282 & 0.958 & 3.859 & 0.325 \\
\hline [Оіг] 3729 & 0.895 & 0.062 & 0.552 & 0.168 & 2.133 & 0.174 & 2.020 & 0.253 & 2.399 & 0.095 & 2.616 & 0.375 & 2.289 & 0.275 \\
\hline [NeIII] 3869 & $\cdots$ & $\cdots$ & $\cdots$ & $\ldots$ & 0.033 & 0.006 & 0.072 & 0.035 & $\cdots$ & $\ldots$ & 0.158 & 0.060 & 0.039 & 0.008 \\
\hline [Оіні] 4363 & $\cdots$ & $\ldots$ & $\cdots$ & $\ldots$ & $\ldots$ & $\ldots$ & 0.005 & 0.008 & $\ldots$ & $\ldots$ & $\ldots$ & $\ldots$ & $<0.002$ & 0.005 \\
\hline HeI 4471 & $\ldots$ & $\ldots$ & $<0.041$ & 0.015 & 0.031 & 0.004 & 0.036 & 0.008 & 0.032 & 0.003 & $\ldots$ & $\ldots$ & 0.031 & 0.006 \\
\hline [OIII] 5007 & $<0.027$ & $\ldots$ & $<0.012$ & $\ldots$ & 0.905 & 0.043 & 1.573 & 0.078 & 0.601 & 0.009 & 1.452 & 0.090 & 1.260 & 0.064 \\
\hline HeI 5876 & 0.004 & 0.002 & $\cdots$ & $\ldots$ & 0.079 & 0.005 & 0.090 & 0.015 & 0.086 & 0.003 & 0.088 & 0.011 & 0.079 & 0.007 \\
\hline [OI] 6300 & $\cdots$ & $\cdots$ & 0.009 & 0.005 & $\ldots$ & .. & $\ldots$ & $\cdots$ & 0.010 & 0.001 & 0.013 & 0.007 & $\cdots$ & $\cdots$ \\
\hline [SIII] 6312 & $\cdots$ & $\ldots$ & . & . & $\cdots$ & .. & $\cdots$ & $\ldots$ & 0.012 & 0.001 & $\cdots$ & & $\cdots$ & $\ldots$ \\
\hline [NII] 6584 & $\cdots$ & $\ldots$ & 0.295 & 0.028 & $\ldots$ & $\ldots$ & $\ldots$ & $\ldots$ & 0.265 & 0.008 & 0.242 & 0.034 & $\ldots$ & $\cdots$ \\
\hline [SII] 6716 & $\cdots$ & $\cdots$ & 0.491 & 0.043 & $\ldots$ & $\ldots$ & $\ldots$ & $\ldots$ & 0.220 & 0.007 & 0.343 & 0.041 & $\ldots$ & $\ldots$ \\
\hline [SII] 6731 & $\ldots$ & $\ldots$ & 0.349 & 0.031 & $\ldots$ & $\ldots$ & $\ldots$ & $\ldots$ & 0.157 & 0.005 & 0.231 & 0.032 & $\ldots$ & $\ldots$ \\
\hline $\mathrm{c}(\mathrm{H} \beta)$ & 0.430 & 0.048 & 0.389 & 0.099 & 0.034 & 0.295 & 0.464 & 0.099 & 0.302 & 0.037 & 0.148 & 0.063 & 0.749 & 0.102 \\
\hline $\log (\mathrm{O} / \mathrm{H})$ & $\cdots$ & $\cdots$ & .. & $\ldots$ & $\ldots$ & .. & -3.4 & 0.5 & $\ldots$ & $\cdots$ & $\ldots$ & $\cdots$ & $\ldots$ & $\ldots$ \\
\hline $\log (\mathrm{N} / \mathrm{O})$ & $\cdots$ & $\ldots$ & $\ldots$ & $\ldots$ & $\ldots$ & $\ldots$ & $\ldots$ & $\ldots$ & -1.42 & $\cdots$ & -1.5 & $\ldots$ & $\ldots$ & $\ldots$ \\
\hline $\log (\mathrm{He} / \mathrm{H})$ & $\ldots$ & $\ldots$ & $\cdots$ & $\ldots$ & -1.21 & 0.01 & -1.17 & 0.02 & -1.19 & 0.02 & -1.20 & 0.02 & -1.12 & 0.02 \\
\hline $\mathrm{H} \beta$ flux ${ }^{d}$ & 7.552 & $\ldots$ & 6.041 & $\ldots$ & 9.125 & $\ldots$ & 27.24 & $\ldots$ & 19.00 & $\ldots$ & 2.035 & $\ldots$ & 28.10 & $\ldots$ \\
\hline
\end{tabular}

Note: ${ }^{a}$ Flux measurements are relative to $\mathrm{H} \beta$.

${ }^{b}$ Objects with data taken on different nights are labeled such that n1 refers to 2008 January 29 and n2 refers to either 30 or 31 January 2008.

${ }^{c}$ There are two slit positions for MCELS L 35 from each of the two nights we observed it.

${ }^{d} \mathrm{The} \mathrm{H} \beta$ fluxes listed here are the fluxes within the $0 .{ }^{\prime \prime} 7$ slit in units of $10^{-14} \mathrm{erg} \mathrm{s}^{-1} \mathrm{~cm}^{-2}$. They are not representative of the $\mathrm{H} \beta$ flux from the entire nebula. 
We assign spectral types (SpT) to the stars from the rectified stellar spectra (Figure 4.2) following the criteria of Walborn \& Fitzpatrick (1990). The spectral type assignments are based on the independent spectral typing by four individuals and are accurate to half a spectral type. The earliest $\mathrm{SpT}$ is O5.5, and the latest is B0.5. Most of the stars are luminosity class V, but our sample also contains two B supergiants (Table 4.1).

In addition, we collect single exposures of $20 \mathrm{~s}$ and $10 \mathrm{~s}$ in filters $B$ and $V$, respectively. We obtain photometry from these images using the IRAF task apphot. Ten of our targets are in common with stars in the OGLE-III survey (Udalski et al., 2008). We compare our measured $V$ magnitudes to the OGLE-III magnitudes and find a mean difference of $-0.07 \mathrm{mag}$ with a standard deviation of $0.15 \mathrm{mag}$. We also have two nebulae, MCELS L 43 and L 52, whose stars are in common with the Massey (2002) UBVR survey of the Magellanic Clouds, and we find agreement in $V$ to 0.01 and 0.09 mag respectively. As a further check, we compare the Massey (2002) and OGLE III $V$ magnitudes for $\sim 50$ stars with $V$ between 13 and 14 mag. The mean difference $\left(V_{\text {Massey }}-V_{\text {OGLEIII }}\right)$ and standard deviation are -0.05 and 0.11 mag, respectively. From this we see that our values are bracketed by the published literature values. We obtain absolute $V$ magnitudes, $M_{V}$, after correcting our observed $V$ for the measured extinction and assuming an LMC distance modulus of 18.48 (Westerlund, 1997).

\subsubsection{OB Companions}

One important consideration is the possible contamination from $\mathrm{OB}$ companions in our sample. The binary fraction for massive stars is thought to be $40-70 \%$ (e.g., Sana et al., 2009, 2011) and possibly as high as 90\% (Kiminki \& Kobulnicky, 2012). Any additional OB stars in the nebula will both contribute to the ionizing photon budget and affect the shape of the ionizing SED. For OB stars, $Q_{0}$ changes by $0.1-0.6$ dex for each full step in SpT (Smith et al., 2002). Thus, the impact of a companion on $Q_{0}$ will not affect the results unless they are within 1-2 SpT of the primary star.

The available data put some constraints on possible companion stars. For the 
objects in our sample, the nearest resolved stars have magnitudes that are $>2$ mag fainter than our target stars, thus ruling out contributions to $Q_{0}$ from companions at large distances from the central star. However, we can still have companions on scales smaller than our spatial resolution, $1 .^{\prime \prime} 6$ which corresponds to $\sim 0.5$ pc. In these cases, the observed magnitudes will include contributions from both stars, if a companion is present. Thus, the luminosities derived from our observed magnitudes and then assigned to the atmosphere models in our simulations should be the sum of the luminosities from all stellar components.

For a binary or companion, one would expect to have spectral type diagnostics representing a mixture of the two spectral types, except in the case of an equal mass binary. None of our stellar spectra show evidence for composite SpT, implying that either any companions are more than two spectral classes later than the dominant star, or that any binary systems must be 'twins'. In the latter case, $Q_{0}$ from the atmosphere models would be around half that of $Q_{0}$ from $L(\mathrm{H} \alpha)$. We discuss $Q_{0}$ in more detail in Section 4.3.3, but we note here that we do not find support for equal mass binaries from the $Q_{0}$ comparison except for MCELS L 351. For that object, the $Q_{0}$ from the atmosphere models is about half that of $Q_{0}$ from $L(\mathrm{H} \alpha)$, and we discuss it in more detail in Section 4.3.4. We note that two of our objects, MCELS L 346 and MCELS L 390, are confirmed eclipsing binaries. However, their companion stars are either much fainter or greater than two SpT later than the primary star, as we discuss further in Section 4.3.4. Thus, while we cannot conclusively rule out binaries from the rest of our sample, the data available suggest that any binaries present will have difference in SpT greater than 1-2 types and, thus, will not significantly affect our results.

\subsubsection{Method}

To evaluate the stellar atmosphere models, we compare the HiI regions in our sample to the predictions of photoionization simulations. As discussed in the Introduction, the line emission from HiI regions depends primarily on the metallicity $(Z)$, the ionization parameter $(U)$, and the SED of the ionizing star. When $Z$ and $U$ are 
constrained, differences between the predictions from photoionization simulations and the observed HiI region spectrum can be directly linked to differences in the shape of the model SED and that of the actual ionizing star.

Photoionization calculations are performed with version 08.00 of CLOUDY (Ferland et al., 1998). To set up the CLOUDY simulations, we set the chemical abundance, inner nebular radius, density, filling factor, and dust abundance to match the nebular abundance and ionization parameter of the observed nebulae. The chemical abundances are set using observed constraints as follows. In MCELS L 28 and L 351, we detect the auroral [OIII] $\lambda 4363$ line. We use the ratio [OIII] $\lambda 4959,5007 /[\mathrm{OIII}]$ $\lambda 4363$ as input for the IRAF task temden to obtain the electron temperature, $T_{e}$. With $T_{e}$ in hand, we derive the oxygen abundance (Table 4.2) using the IRAF task ionic. For this calculation we assume $T_{e}([\mathrm{OII}])=T_{e}([\mathrm{OIII}])$. To set the abundance of S, C, Ne, Ar, Si and Fe, we use the relations from McGaugh (1991), which relate the elemental abundances to that of oxygen. For the rest of our objects, [OIII] $\lambda 4363$ falls in the gap between CCD chips. In these cases, we adopt the mean LMC abundances measured by Garnett (1999). For all our objects, except for MCELS L 346, L 351, and DEM L 283b, we calculate the nitrogen abundance from the ratio [NII]/[OII], as described in Pérez-Montero \& Díaz (2005). Those values are shown in Table 4.2. We note that except for MCELS L 345, the mean difference between the calculated $\log (\mathrm{N} / \mathrm{O})$ and the Garnett (1999) values is 0.17 dex with a standard deviation of 0.11 dex. This is comparable to the uncertainty in both the relation (Pérez-Montero \& Díaz, 2005) and in the measured abundances above.

The mean ionization parameter for constant density models is defined by

$$
U \equiv \frac{Q_{0}}{4 \pi c n_{e} R_{S}^{2}} \sim\left(Q_{0} n \epsilon^{2}\right)^{\frac{1}{3}},
$$

where $Q_{0}$ is the rate of H-ionizing photons, $c$ is the speed of light, $n_{e}$ is the electron density, $R_{S}$ is the radius of the Strömgren sphere and $\epsilon$ is the filling factor of the gas (McGaugh, 1991). Essentially, $U$ describes the ionizing photon density relative to the gas density. The ionization parameter will depend on both the type of star 
producing the ionizing radiation and the gas distribution around the star. As can be seen in Figure 4.1, the nebulae are Strömgren spheres, which means we can assume simple spherical geometry for the simulated nebulae. This assumption is further supported by the observed line ratios. Specifically, if these nebulae were face-on blister HII regions instead of Strömgren spheres, we would expect the simulations to over-predict $[\mathrm{OII}] / \mathrm{H} \beta$ and $[\mathrm{NII}] / \mathrm{H} \beta$ relative to the observed line ratios (Morisset et al., 2005; Pellegrini et al., 2012). However, we see the opposite behavior, as discussed in Section 4.3.1.

The nebular parameters that control $U$ are those that describe the radial density profile of the nebula: the initial $\left(R_{\text {inner }}\right)$ and outer $\left(R_{\text {nebula }}\right)$ radii of the cloud, hydrogen density $\left(n_{\mathrm{H}}\right)$, and $\epsilon$. We use a combination of emission-line diagnostics and the MCELS $\mathrm{H} \alpha$ images to set these values. To constrain the density distribution, we examine the MCELS images and the $\mathrm{H} \alpha$ and [OIII] $\lambda 5007$ line profiles along the slit. The [OIII $\lambda 5007$ and $\mathrm{H} \alpha$ profiles indicate that $R_{\text {inner }}$ is between $0.1 \times R_{\text {nebula }}$ and $0.5 \times R_{\text {nebula }}$. We measure $R_{\text {nebula }}$ from the $\mathrm{H} \alpha$ MCELS images of the nebulae and use those values to set the inner and outer radii in the CLOUDY simulations.

To obtain $n_{\mathrm{H}}$, we assume $n_{\mathrm{H}}=n_{e}$, since hydrogen is fully ionized in a typical HıI region. The [OII] $\lambda \lambda 3726,3729$ and [SII] $\lambda \lambda 6716,6731$ density-sensitive doublets both indicate that nearly all our objects are in the low-density regime $\left(n_{e}<100 \mathrm{~cm}^{-3}\right)$ below which these diagnostics are no longer sensitive to the density. We also estimate the density using the $\mathrm{H} \alpha$ emission measure based on the $\mathrm{H} \alpha$ photometry, and find $n_{e} \lesssim 30 \mathrm{~cm}^{-3}$, which is consistent with our limit from the [SII] and [OII] diagnostics. Based on these constraints, we use $n_{\mathrm{H}} \leq 100 \mathrm{~cm}^{-3}$ as an upper limit for the densities in all our simulations. Most of the profiles are consistent with a flat density profile, either with or without a central hole. We therefore assume a uniform density in the CLOUDY simulations. In Section 4.3.2 we explore the effects of $\epsilon<1$. Finally, the HII regions in our sample are optically thick (Pellegrini et al., 2012), and we set $n_{\mathrm{H}}$ and $\epsilon$ such that the ionizing photons are absorbed within the observed nebular size (Table 4.1).

In addition to the properties discussed above, dust will affect the nebula. In 
particular, photoelectric heating from dust can contribute as much as $30 \%$ of the total heating (van Hoof et al., 2004). Therefore, we include both graphite and silicate dust in our simulations. We adopt LMC gas distributions and a dust-to-gas ratio, $A_{V} / N(\mathrm{HI})=1.2 \times 10^{-22} \mathrm{mag} \mathrm{cm}^{2}$, consistent with Weingartner \& Draine (2001), which were introduced into CLOUDY in Pellegrini et al. (2011). Our simulated nebulae also include $5 \mathrm{~km} \mathrm{~s}^{-1}$ turbulence. Finally, since our observations are based on long slit spectroscopy, we implement the CLOUDY command slit, which predicts the line ratios that would be observed through a slit across the simulated nebula.

We run CLOUDY simulations with the same set of nebular parameters $\left(R_{\text {inner }}\right.$, $n_{e}, \epsilon, \log (\mathrm{O} / \mathrm{H})$, dust) for each stellar atmosphere model. The simulations, which run radially, are stopped when the nebular temperature drops to $1000 \mathrm{~K}$. In addition to specifying which atmosphere grid to use for the source, we must also assign the luminosity and $T_{\text {eff }}$ of the star. We calculate stellar luminosity from $M_{V}$ using

$$
\log L / L_{\odot}=0.4 \times\left(M_{\mathrm{bol}, \odot}-\left(M_{V}+\mathrm{BC}\right)\right),
$$

where $M_{\mathrm{bol}, \odot}$ is the solar bolometric magnitude and $\mathrm{BC}$ is the bolometric correction as a function of $T_{\text {eff }}$ from Martins et al. (2005), except for the case of MCELS L 345, the B0.5 Iab star, where we use the bolometric correction from Crowther et al. (2006). The SpT $-T_{\text {eff }}$ conversion is model-dependent and we leave the $T_{\text {eff }}$ as a free parameter in our simulations within a range motivated by the $\mathrm{SpT}$ of the star. We note that since the $\mathrm{BC}$ becomes increasingly negative with increasing $T_{\text {eff }}$, the input stellar luminosity is recalculated for each input $T_{\text {eff }}$ to maintain consistency with the observed $M_{V}$. For all our dwarf stars, we assume $\log (g)=4.0$.

We determine the best $T_{\text {eff }}$ for each star to be the one that produces the correct balance of ionizing flux at both high and low energies. We accomplish this task by plotting the predicted emission-line intensity as a function of ionization potential of the ion species. An example is shown in Figure 4.4, in which we plot the results of six simulations that differ in $T_{\text {eff }}$. As expected, as $T_{\text {eff }}$ decreases from $43,000 \mathrm{~K}$ to $35,000 \mathrm{~K}$, the predictions for $[\mathrm{OII}] / \mathrm{H} \beta$ and $[\mathrm{NII}] / \mathrm{H} \beta$ increase, while the predictions 


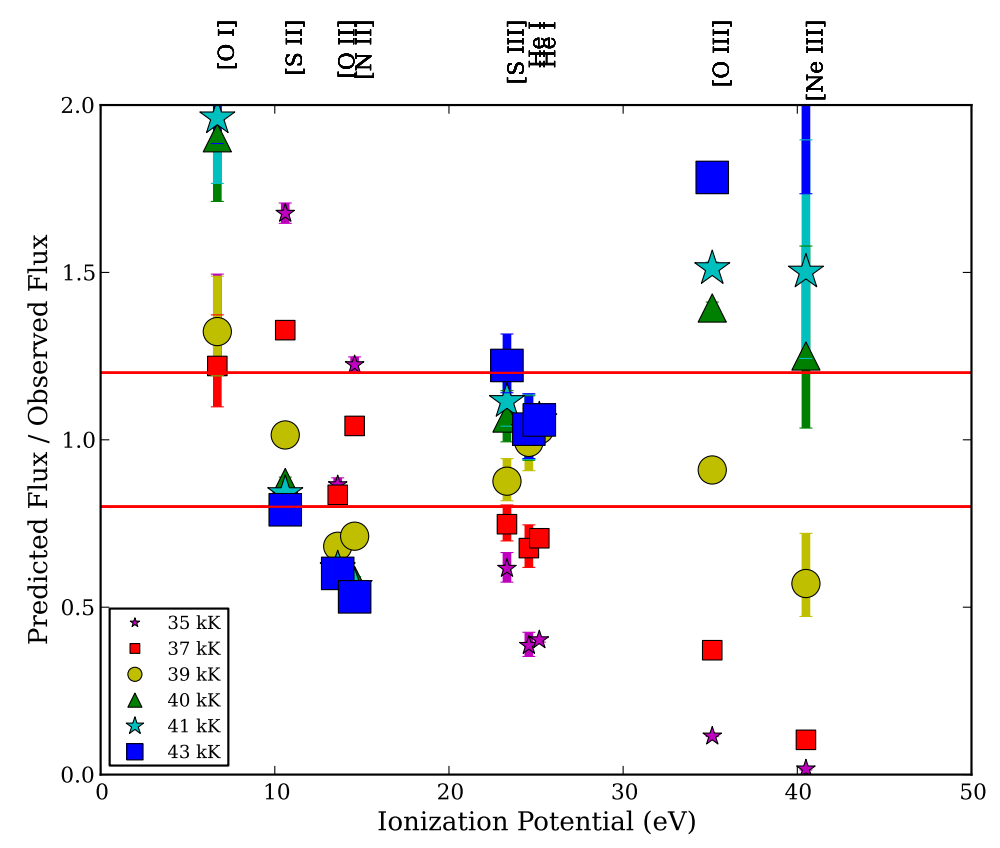

Figure 4.4. Ratio between the predicted and observed emission-line intensities for MCELS L 28. The simulations shown above are ionized by WM-basic atmosphere models ranging from $T_{\text {eff }}=$ 35,000 to $43,000 \mathrm{~K}$. The red horizontal lines are at $\pm 20 \%$ and are representative of the observational variance. The best-fitting model is defined as the one with the flattest overall slope, with most points lying between the red lines. In this case, $T_{\text {eff }}=39,000 \mathrm{~K}$ (yellow circles) has the best fit.

for $[\mathrm{OIII}] / \mathrm{H} \beta$ and $[\mathrm{NeIII}] / \mathrm{H} \beta$ decrease. Thus, the overall slope of the points decreases with decreasing $T_{\text {eff }}$. We define the optimal model at the turnover $T_{\text {eff }}$, where the slope is flat. For the models shown in Figure 4.4, this would be around $T_{\text {eff }}=39,000$ $\mathrm{K}$ (yellow circles). We also prioritize models that match the observed $\mathrm{HeI} / \mathrm{H} \beta$ lines in our spectrum. The ratio of the HeI to Hi recombination lines reflects the level of He ionization in the nebula. As long as He remains partially ionized, this ratio will be primarily dependent on the SED of the ionizing source (Kennicutt et al., 2000). We note that we do not detect HeII $\lambda 4686$ in any of our spectra. Therefore, we can set an upper limit on $T_{\text {eff }}$ by requiring a non-detection of that line in the simulations as well. 


\subsection{Photoionization Models}

\subsubsection{Uniform Density Models}

Figure 4.1 shows that our HII regions, to first approximation, are simple Strömgren spheres. We use the method described in Section 4.2.3 and the models described in Section 4.1.1 to first generate a grid of model Hir regions with $\epsilon=1$. Figures 4.5 and 4.6 show the results using this prescription for MCELS L 28 and L 43, which are ionized by an $\mathrm{O} 5.5 \mathrm{~V}$ and an $\mathrm{O} 8 \mathrm{~V}$ star, respectively. These figures are representative of the results from the rest of the sample. The left panels of the figures show $[\mathrm{OIII}] \lambda 5007 / \mathrm{H} \beta$ versus $[\mathrm{OII}] \lambda 3727 / \mathrm{H} \beta$, which reflects variations in $U$. In nebulae with high $U$ there will be more [OIII] relative to [OII], and the points will occupy the top left of the plot. As $U$ decreases, [OII] becomes stronger relative to [OIII], and the points will move toward the lower right. The right panels show [OII] $\lambda 3727 / \mathrm{H} \beta$ versus HeI $\lambda 5876 / \mathrm{H} \beta$. This panel illustrates how well [OII] is predicted at the appropriate $T_{\text {eff }}$, which is traced by HeI $/ \mathrm{H} \beta$ (Kennicutt et al., 2000). The solid and dotted lines show how the line ratios change when the $R_{\text {inner }}$ and $T_{\text {eff }}$ are changed, respectively.

It is immediately apparent that these models do not reproduce the observed emission-line ratios. In the simulations using CoStar and WM-basic atmosphere models, the simulation tracks generally run below and to the left of the observed values (Figure 4.5; left panel). This indicates that not enough [OII] is produced when [OIII] is well predicted and suggests that $U$ is too high. Furthermore, from the righthand plots, we see that simply changing the $T_{\text {eff }}$ is not a viable solution; the predicted [OII] flux does not match the observations for the entire range in $T_{\text {eff }}$. This is the case for all our objects except for MCELS L 43 (Figure 4.6) and MCELS L 344 (not shown). In contrast, the simulated nebulae ionized by TLUSTY atmospheres have lower ionization parameters. The softer TLUSTY SED is able to reproduce the observed [OIII]/H $\beta$ and [OII]/H $\beta$ line ratios in MCELS L 28 (Figure 4.5), MCELS L 351, and MCELS L 394. However, it does not reproduce the line ratios for the other emission lines in the spectrum. For the rest of the objects, the TLUSTY SED is too soft, and the simulations have an ionization parameter that is too low (Figure 4.6). 


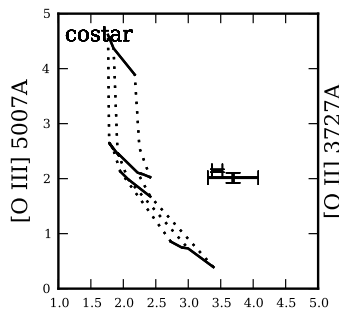

[O II] 3727A

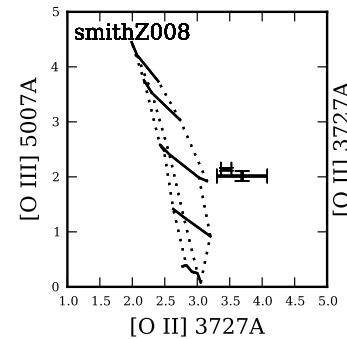

[O II] 3727A

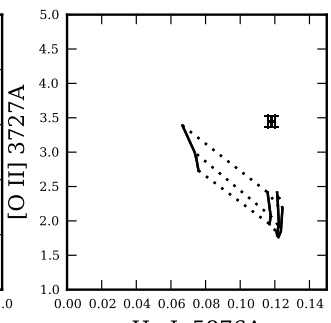

He I $5876 \mathrm{~A}$

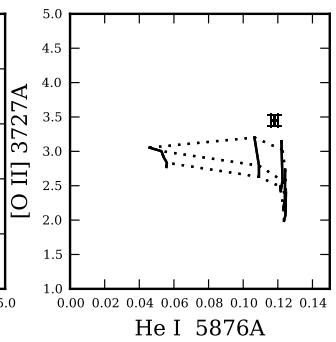

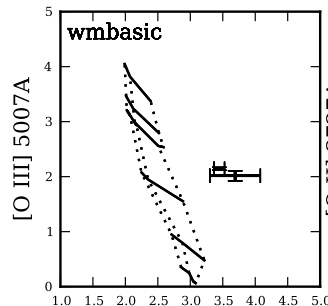

[O II] 3727A

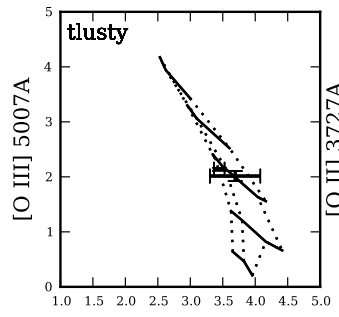

[O II] 3727A

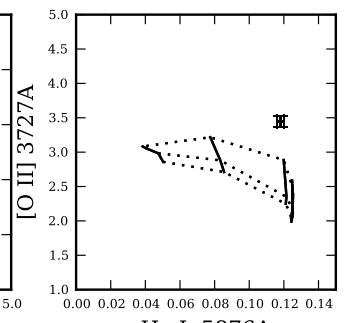

He I $5876 \mathrm{~A}$

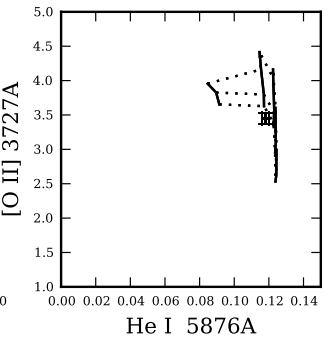

Figure 4.5. Predicted line strengths of [OIII] vs. [OII] and [OII] vs. HeI for MCELS L 28 (O5.5 $\mathrm{V})$. Line strengths are plotted relative to $\mathrm{H} \beta$. The solid lines represent models with the same $T_{\text {eff }}$, but changing $R_{\text {inner }}$. The effective temperatures shown are $T_{\text {eff }}=35,37,39,41$, and $43 \mathrm{kK}$ and increase from the bottom towards the top and from left to right, for [OIII] vs. [OII] and [OII] vs. HeI, respectively. Note, the CoStar plot only goes up to $T_{\text {eff }}=41 \mathrm{kK}$. Dotted lines denote models with the same $R_{\text {inner }}$ but changing $T_{\text {eff }}$. These lines correspond to $0.10,0.25$, and $0.50 R_{\text {nebula }}$, with the radius increasing towards the right in [OIII] vs. [OII] and toward the top in HeI vs. [OII]. For comparison, the observed value is shown by the black point.

We rule out the adopted metallicity as the source of the discrepancy between the simulations and observations, as follows. The metallicity of the gas strongly affects the observed line ratios. As the metallicity decreases, there are fewer metals to provide cooling, and the nebula is hotter. Thus, the under-prediction of the low ions seen above could indicate that the adopted metallicities are too low. To explore how this might affect our results, we generate a CLOUDY grid for MCELS L 28 in which we change the metallicity from $\log (\mathrm{O} / \mathrm{H})=-3.4$ to $\log (\mathrm{O} / \mathrm{H})=-3.9$. The results from this grid for the $[\mathrm{OII}] / \mathrm{H} \beta$ and $[\mathrm{OIII}] / \mathrm{H} \beta$ ratios are shown in Figure 4.7. The measured $\log (\mathrm{O} / \mathrm{H})=-3.51$ for MCELS L 28.

We find that changing the metallicity will not reconcile the discrepancy in the low ions. Although increasing the metallicity does decrease the predicted [OIII]/[OII] ratio for a given $T_{\text {eff }}$ (Figure 4.7(a)), it does so by decreasing [OIII]/H $\beta$ (Figure 4.7(b)) Meanwhile, $[\mathrm{OII}] / \mathrm{H} \beta$ remains under-predicted for the whole range of metallicities (Figure $4.7(\mathrm{c})$ ). Therefore, even though changing the metallicity might bring the overall $[\mathrm{OIII}] /[\mathrm{OII}]$ closer to the observed, it cannot account for the discrepancies 


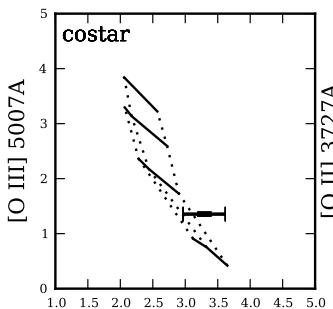

[O II] 3727A
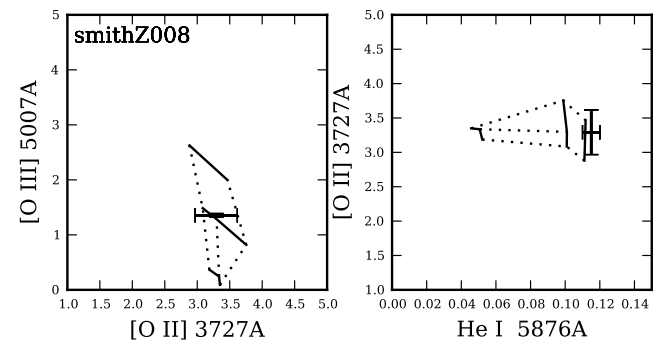

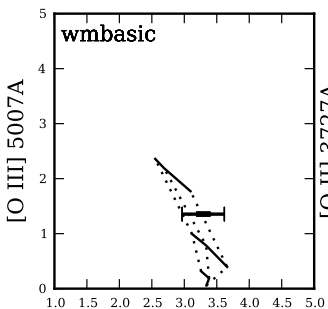

[O II] 3727A

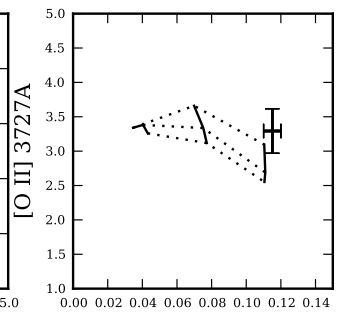

He I 5876A

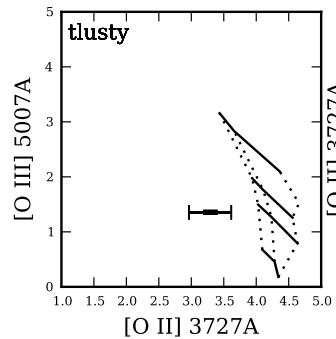

[O II] 3727A

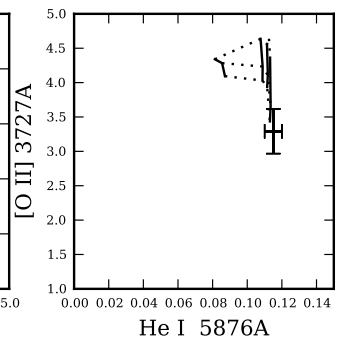

Figure 4.6. Same as Figure 4.5 but for MCELS L 43 (O8 V). The inner radii shown are 0.10, 0.25, and $0.50 R_{\text {nebula }}$. The effective temperatures are $35,37,39,40 \mathrm{kK}$.

between the simulations and the observations for the individual lines. We note that decreasing $\log (\mathrm{O} / \mathrm{H})$ by 0.1 dex, from the observed to the mean LMC value, will change the $T_{\text {eff }}$ required to reproduce the observed emission-line fluxes by $500 \mathrm{~K}$. This is comparable to the $250-500 \mathrm{~K}$ uncertainty in determining $T_{\text {eff }}$ using the emissionline ratios. Since we do not have measured metallicities for most of the objects in our sample, this $500 \mathrm{~K}$ is included in the error on the best fitting $T_{\text {eff }}$, discussed below.

Another possible cause of the general under-prediction of the low ions is the nebular structure. In this case, there is not enough dense gas in our simulations at large radii to receive a diluted radiation field, which is needed to reproduce the emission from lines with low ionization potential. This could be because $R_{\text {inner }}$ is set too close to the star, or because the nebula has a clumpy distribution of denser gas. An increase of $R_{\text {inner }}$ increases the [OII] and [NII] flux, while it simultaneously suppresses the [OIII] and [NeIII] flux. At face value, this would move the predictions closer to the observations. However, even if we vary the inner radius from 0.1 to $0.5 R_{\text {nebula }}$, we still are not able to simultaneously reproduce the two sets of lines (Figure 4.5). Given that the inner radius is constrained to be $\lesssim 0.25 R_{\text {nebula }}$ in all our objects, except DEM L 283b, $R_{\text {inner }}$ set too close to the star cannot explain the discrepancy in the low ions. Therefore, neither the metallicity nor $R_{\text {inner }}$ can explain the discrepancy 


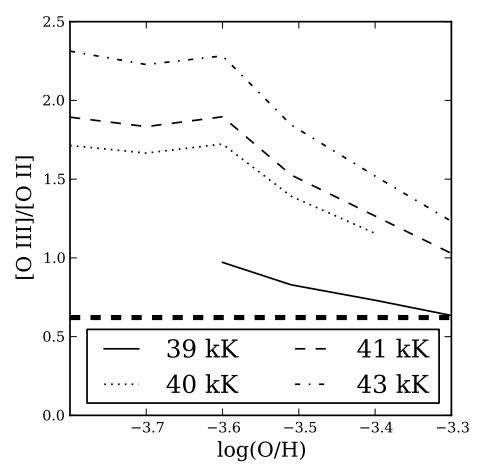

(a)

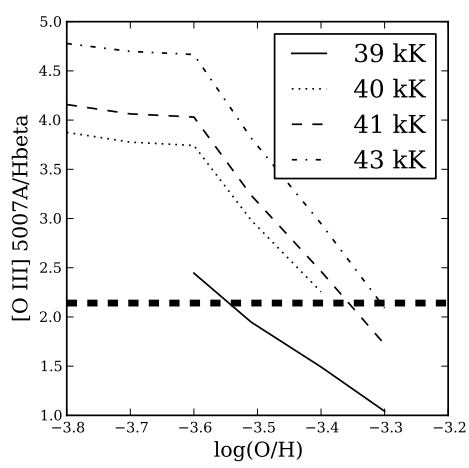

(b)

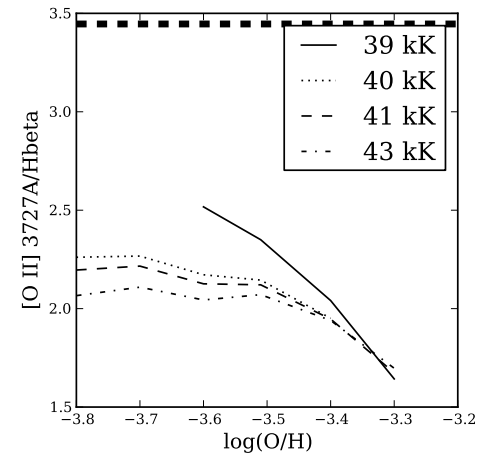

(c)

Figure 4.7. Predicted emission-line ratios of $[\mathrm{OIII}] \lambda 5007 / \mathrm{H} \beta$ and $[\mathrm{OII}] \lambda 3727 / \mathrm{H} \beta$ as a function of metallicity are shown for simulations of MCELS L 28 ionized by WM-basic atmospheres with $T_{\text {eff }}=$ $39-43 \mathrm{kK}$ (thin lines). The nebular parameters for these models are: $n_{e}=30 \mathrm{~cm}^{-3}, \epsilon=1.00$, and $R_{\text {inner }}=0.10 R_{\text {nebula }}$. These are compared to the observed values for MCELS L 28 (thick dashed line) for which the observed metallicity is $\log (\mathrm{O} / \mathrm{H})=-3.51$.

in these simple Strömgren sphere models. In the next section, we explore the effects of a clumpy gas distribution.

\subsubsection{Density Clumps}

In Hir regions, non-uniform gas distributions are often invoked to explain the observed properties. In fact, direct imaging of HiI regions reveals complex morphology, which includes density gradients and/or clumps and filaments with high density contrast. Non-uniform gas density has long been invoked to explain observed parameters (e.g., Osterbrock \& Flather, 1959; Viegas \& Clegg, 1994; Williams, 1992; Liu \& Danziger, 1993). One example is the long-standing problem reconciling the discrepant values for $T_{e}$ that result from different diagnostics. This has been of particular concern 


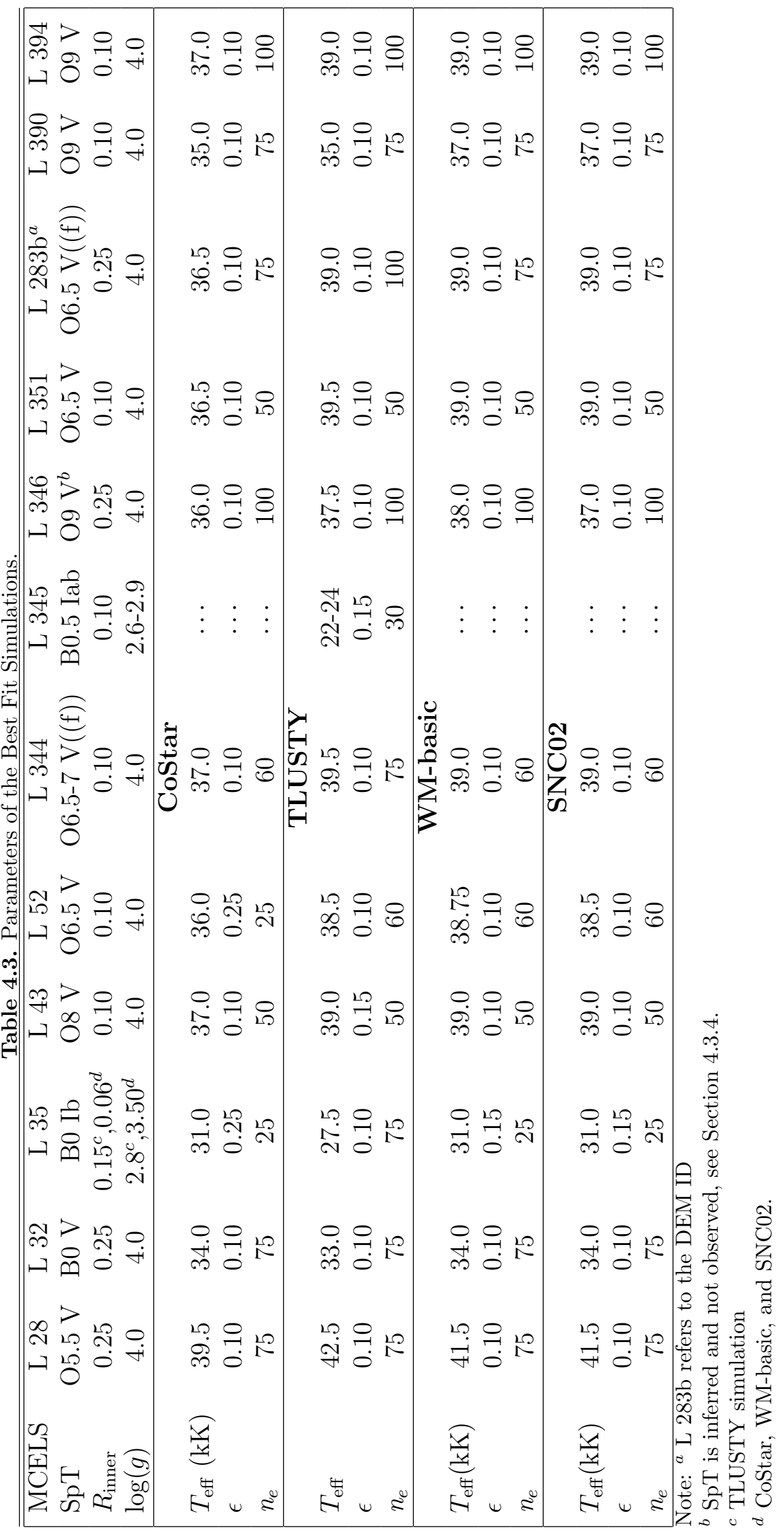


because the uncertainty in $T_{e}$ translates into significant uncertainty in the derived abundances. One suggestion is that this problem arrives from temperature fluctuations in the ionized gas, which are described by the $t^{2}$ parameter (Peimbert, 1967). These fluctuations could arise from small clumps of dense gas within the nebulae (e.g., Viegas \& Clegg, 1994; Williams, 1992; Liu \& Danziger, 1993) or from fluctuations in chemical abundance rather than density (Rubin, 1989; Kingdon \& Ferland, 1995; Giammanco et al., 2004; Tsamis \& Péquignot, 2005).

In HiI regions, non-uniform gas distributions are often invoked to explain the observed properties. In fact, direct imaging of Hiı regions reveals complex morphology, which includes density gradients and/or clumps and filaments with high density contrast. Non-uniform gas density has long been invoked to explain observed parameters (e.g., Osterbrock \& Flather, 1959; Viegas \& Clegg, 1994; Williams, 1992; Liu \& Danziger, 1993). We therefore explore how changing the density structure affects line emission by considering a clumpy medium. For our analysis here, we assume that dense clumps of gas are uniformly spread throughout the nebula, with a vacuum between them (Osterbrock \& Flather, 1959). In reality, the clumps will be interspersed with lower density, diffuse gas. However, the emission measure is proportional to $n_{e}^{2}$. Therefore, most of the observed flux is coming from the dense clumps, and we can treat the inter-clump space as though it were a vacuum. In the simulations, these clumps are treated in a statistical sense; both the volume emissivity of the gas and the optical depth along the line of sight are decreased by $\epsilon$ in each zone calculated by the code (Ferland et al., 1998). This effectively means that the volume of the emitting material in a given zone is decreased by $\epsilon$ and that there is a factor of $\epsilon$ less material available to absorb the incident radiation.

In our final simulation grid, we vary $T_{\text {eff }}$ and $\epsilon$, with the density chosen as discussed above. A decrease in the filling factor of the gas necessitates an increase in the density of the gas to maintain the nebular radius (Equation 4.2). In this scenario, dense gas is present at small radii to experience a strong radiation field, as required for the lines with high ionization potential. At the same time, more dense gas is present at larger radii from the star that receives a diluted radiation field to reproduce ions with low 
ionization potential. Our constraints on the sizes of the simulated nebulae, combined with our upper limit on the density, effectively provide us with lower limits for $n_{\mathrm{H}}$ and $\epsilon$. We cannot decrease the densities past the values that lead to correctly sized nebulae when $\epsilon=1.0$, and we cannot decrease $\epsilon$ below the value that corresponds to $n_{\mathrm{H}}=100 \mathrm{~cm}^{-3}$. We note that the filling factor set by the density limit, $\epsilon \sim 0.10$ in most of our objects, is consistent with the $10^{-1}-10^{-3}$ commonly used in the literature (Kennicutt, 1984; Giammanco et al., 2004; Hunt \& Hirashita, 2009). Furthermore, the nebulae in our sample exhibit substructure (Figure 4.1) that is consistent with this degree of clumping.

The simulations using $\epsilon<1$ are able to reproduce ions with both low and high ionization potential simultaneously. Table 4.3 shows the parameters of the best fitting model for each nebula. These parameters include $R_{\text {inner }}$ and $\log (g)$ for each nebula and star, as well as the $T_{\text {eff }}, \epsilon$, and $n_{\mathrm{H}}$ that correspond to the best simulation for each atmosphere model. Figure 4.8 shows the results of these simulations. In this Figure, we plot the ratio of predicted emission-line strengths, relative to $\mathrm{H} \beta$, to the observed, $F_{\text {pre }} / F_{\text {obs }}$, as a function of the ionization potential of the emission line. Each model atmosphere corresponds to a different color and symbol. The error bars represent the observational error, which includes the line measurement, reddening correction and flux calibration errors. As discussed in Section 4.2.3, these simulations are selected by finding the combination of free parameters $\left(\epsilon, T_{\text {eff }}\right)$ that reproduces the observed HeI emission-lines, and yields the flattest slope with most points lying within $\pm 20 \%$ of unity, denoted by the red horizontal lines. The $20 \%$ takes into account the observational variance, which is estimated from MCELS L 28, L 32, L 35 and L 344, for which we have multiple nights of data and/or multiple slit positions (Figures 4.8(a), 4.10(a), 4.10(b), and 4.9(a)). The additive error on our selected $T_{\text {eff }}$ and $\epsilon$ for a given $R_{\text {inner }}$ is $\sim 500-1000 \mathrm{~K}$ and 0.1 , respectively. The error for $T_{\text {eff }}$ includes the contribution based on the uncertainty in our metallicity and density assumptions, as discussed in Section 4.2.3 and Section 4.3.1.

Thus far, we have considered a density profile that is constant with radius, while including density clumps. However, three objects, MCELS L 032, MCELS L 052, and 
MCELS L 390, show evidence for significant density gradients from their azimuthally averaged $\mathrm{H} \alpha$ surface brightness profiles. To explore the effect this has on our results, we model MCELS L 052, the object with the steepest density gradient, with a radial density profile tailored to match the azimuthally averaged $\mathrm{H} \alpha$ surface brightness profile. On average, the simulated emission lines that are predicted using the radial density profile agree with the observations to within $15 \%$. This is the consistent with the constant density simulation, except for [NeIII] intensities, which are underpredicted by $10 \%$ in the radial density model and overpredicted by the same amount in the constant density models (Figure 4.8(b)). The best fitting simulations to the observed emission lines in the radial density grid have $\epsilon$ and $T_{\text {eff }}$ that are within $3 \%$ and $\sim 500 \mathrm{~K}$ of the results from the constant density models, which is within the error for both those properties. The agreement between the two nebular treatments confirms that fitting the observed, integrated emission-line spectra indeed yield a robust estimate of the ionizing $T_{\text {eff }}$. The mean observed properties are necessarily representative of the nebular conditions, and so the mean gas density and mean nebular excitation yield the appropriate characteristic $T_{\text {eff }}$ exciting the nebula.

On the other hand, [OI] $\lambda 6300$ and [SII] $\lambda 6716,6731$ are not good diagnostics for distinguishing between the SEDs, since they are primarily dependent on nebular density and morphology, and less so on the particular atmosphere chosen. This can be seen in Figure 4.11, where we have plotted the predictions for HeI $\lambda 5876 / \mathrm{H} \beta$ against those of $[\mathrm{OI}] / \mathrm{H} \beta$ and $[\mathrm{SII}] / \mathrm{H} \beta$ from a simulation grid that varies $\epsilon$ and $T_{\text {eff }}$ for MCELS L 28. Given the same nebular conditions, [OI] and [SII] are insensitive to different ionizing SEDs. This is particularly true for $[\mathrm{SII}] / \mathrm{H} \beta$, which changes by up to $25 \%$ for a large range in $T_{\text {eff }}$. A similar behavior is observed for $[\mathrm{OI}] / \mathrm{H} \beta$, although we do see significant changes in $[\mathrm{OI}] / \mathrm{H} \beta$ at high $T_{\text {eff }}$. This might be explained by their relevant excitation mechanisms. In the case of [SII], the ionization potential of this line is $10.60 \mathrm{eV}$, which is below that of $\mathrm{H}$. In this regime, the differences between atmosphere models are small, and so the line will be more sensitive to changes in the gas density and morphology. [OI] $\lambda 6300$, on the other hand, is produced via chargeexchange and, in the conditions of a typical nebula, depends critically on the density 
of $\mathrm{H}^{+}$(Osterbrock \& Ferland, 2006).

\subsubsection{Rate of Ionizing Photons}

One important comparison is between the rate of ionizing photons inferred from the observed nebulae, $Q_{0, \mathrm{H} \alpha}$, and that of the best fitting atmosphere model, $Q_{0, \mathrm{SED}}$. To calculate $Q_{0, \mathrm{H} \alpha}$, we obtain $\mathrm{H} \alpha$ photometry from the MCELS, $\mathrm{H} \alpha$ emission-line image (Table 4.1). These values are corrected for reddening using the reddening corrections from Table 4.2. The rate of ionizing photons calculated from this photometry is listed in Column 2 of Table 4.4. Since the objects in our sample are optically thick, $Q_{0, \mathrm{H} \alpha}$ is a good representation of the $Q_{0}$ emitted by the star. We note that the MCELS narrowband images are not continuum subtracted. As a result, the error on $Q_{0, \mathrm{H} \alpha} \sim 20 \%$ (Pellegrini et al., 2012). Columns 3-6 show the ratio of $Q_{0, \mathrm{SED}}$ and the measured $Q_{0, \mathrm{H} \alpha}$.

It is immediately apparent that the $Q_{0, \mathrm{SED}}$ for dwarf stars is offset from $Q_{0, \mathrm{H} \alpha}$ by $+5 \%,-12 \%,-12 \%$, and $-37 \%$ on average for TLUSTY, WM-basic, SNC02, and CoStar, respectively. This excludes MCELS L394, for which $Q_{0, \text { SED }}$ is $3-5$ times higher than $Q_{0, \mathrm{H} \alpha}$ (see Section 4.3.4), and the two supergiants. From this diagnostic, TLUSTY, best represents the ionizing source. However, both WM-basic and SNC02 also do well, particularly within the $20 \%$ error on the $\mathrm{H} \alpha$ photometry. We note that the offset does depend on atmosphere model, but we find no trend with spectral type or $A_{V}$.

One important factor that affects this comparison is the assumed gravity. In our simulations, we use $\log (g)=4.0$ for all luminosity class V stars. However, these stars may have $\log (g)$ in the range from 3.8 to 4.1 (e.g., Herrero et al., 1999; Massey et al., 2005; Martins et al., 2005). Decreasing $\log (g)$ by just 0.1 dex can increase $Q_{0, \text { SED }}$ by as much as a factor of 2 . This change more than compensates for any discrepancy shown in Table 4.4, and supports that $Q_{0, \mathrm{SED}}$ is consistent with $Q_{0, \mathrm{H} \alpha}$, within the uncertainty.

We note that the chosen $T_{\text {eff }}$ will affect $Q_{0, \mathrm{SED}}$. Both the bolometric correction and the shape of the SED play a role in determining $Q_{0}$, and both properties depend 
on $T_{\text {eff }}$. As noted in Section 4.3.2, the error on $T_{\text {eff }}$ is between 500 and $1000 \mathrm{~K}$. If our chosen $T_{\text {eff }}$ is too low by $1000 \mathrm{~K}$, the appropriate $Q_{0, \text { SED }}$ should be $10 \%-15 \%$ higher than the values from Table 4.4, through a combination of an increased BC and a harder SED. We note that this effect will have a much smaller impact than uncertainty in $\log (g)$, and that a change in $T_{\text {eff }}$ of $1000 \mathrm{~K}$ significantly affects the ionization structure of the nebula, which worsens many of the fits shown in Figure 4.8. Thus, uncertainty in $T_{\text {eff }}$ should not significantly contribute to any offset in $Q_{0, \mathrm{SED}} / Q_{0, \mathrm{H} \alpha}$.

In addition to the general offsets in $Q_{0, \mathrm{SED}} / Q_{0, \mathrm{H} \alpha}$, we also find differences between model grids. In general, TLUSTY has the highest $Q_{0, \mathrm{SED}} / Q_{0, \mathrm{H} \alpha}$, with most values near unity, and CoStar has the lowest, with many values near 0.6. WM-basic and SNC02 fall between them. The trend between atmosphere models reflects the important role that the relative hardness of the SED plays in the temperature selection process, which in turn affects $Q_{0}$. For a given effective temperature, different atmosphere models will produce different $Q_{0}$ (e.g., Voges et al., 2008; Simón-Díaz \& Stasińska, 2008). On top of that, we are sensitive to the shape of the ionizing SED because our atmosphere-model selection criteria depend on matching the emissionline ratios. This means that for a softer atmosphere model (e.g., TLUSTY), we select simulations with higher effective temperatures than we would for simulations using harder atmosphere models (e.g., CoStar). This effect leads to the trend seen above: the best-fitting models for TLUSTY have higher $Q_{0}$ than those of CoStar. Therefore, while the systematic offset in $Q_{0}$ is most likely caused by uncertainty in $\log (g)$, it may also suggest that the WM-basic, SNC02, and CoStar atmosphere models are generally harder than the observed stars. However, we note that this is opposite from what we find based on the $[\mathrm{NeIII}] / \mathrm{H} \beta$ line ratio, as we discuss in Section 4.4.1.

\subsubsection{Individual Objects}

In this section we briefly comment on the individual objects in our sample. Unless otherwise noted, we assume the mean LMC abundances from Garnett (1999). Table 4.2 lists the Helium abundances, which we calculate from the HeI lines using the 
Table 4.4. $Q_{0}$ Comparison.

\begin{tabular}{|ccc|cccc|}
\hline & & \multicolumn{4}{c|}{$Q_{0, \mathrm{SED}} / Q_{0, \mathrm{H} \alpha}$} \\
MCELS & SpT & $Q_{0, \mathrm{H} \alpha}$ & CoStar & TLUSTY & SNC02 & WM-basic \\
\hline L 28 & O5.5 V & 48.94 & 0.51 & 0.95 & 0.67 & 0.71 \\
L 32 & B0 V & 47.91 & 1.01 & 1.27 & 1.08 & 0.84 \\
L 35 & B0 Ib & 48.20 & 0.95 & 2.40 & 1.62 & 0.95 \\
L 43 & O8 V & 48.47 & 0.53 & 0.97 & 0.79 & 0.74 \\
L 52 & O6.5 V & 48.90 & 0.39 & 0.80 & 0.64 & 0.62 \\
L 344 & O7-6.5 V((f)) & 49.02 & 0.54 & 0.99 & 0.80 & 0.75 \\
L 345 & B0.5 Iab & 48.18 & $\ldots$ & $0.16-0.47$ & $\ldots$ & $\ldots$ \\
L 346 & O9 V & 48.48 & 1.06 & 1.84 & 1.27 & 1.36 \\
L 351 & O6.5 V & 49.37 & 0.27 & 0.55 & 0.42 & 0.39 \\
L 390 & O9 V & 48.65 & 0.66 & 1.01 & 1.16 & 1.60 \\
L 394 & O9 V & 47.99 & 2.94 & 5.36 & 4.37 & 4.08 \\
L 283b & O6.5 V((f)) & 48.51 & 0.70 & 1.44 & 1.10 & 1.02 \\
\hline
\end{tabular}

Note: ${ }^{a}$ Eclipsing binary for which the SpT of the ionizing source inferred and not observed, see Section 4.3.4.

${ }^{b}$ Refers to the DEM catalog number.

relations from Benjamin et al. (2002). As a reference, Table 4.3 shows the parameters of our best fitting models.

MCELS L 28 is ionized by OGLE-III 8225 (Udalski et al., 2008), which is the earliest spectral type in our sample, an O5.5 V star (Figures 4.1(a) and 4.8(a)). In this nebula, we detect the auroral [OIII] $\lambda 4363$ emission line, from which we derive $\log (\mathrm{O} / \mathrm{H})=-3.51$. The simulations with the best fit have $\epsilon=0.10$ and $n_{\mathrm{H}}=75 \mathrm{~cm}^{-3}$. As noted in Section 4.3.1, based only on the oxygen lines, the TLUSTY simulation shows a good fit using $\epsilon=1.0$ and $n_{\mathrm{H}}=15 \mathrm{~cm}^{-3}$. However, for that simulation, both [NeIII] and [NII] are under-predicted. Figure 4.8(a) clearly shows this is not the case in the $\epsilon=0.10$ models. If we had selected the $\epsilon=1$ model as our final best fit, the corresponding $T_{\text {eff }}$ would be $39,000 \mathrm{~K}$. This is $3,500 \mathrm{~K}$ cooler than the adopted $T_{\text {eff }}=42,500 \mathrm{~K}$ selected with the lower filling factor. This $T_{\text {eff }}$ difference highlights the importance of using as many emission-line diagnostics as available to determine the fits. The best fitting $T_{\text {eff }}$ are 39,500, 42,500, 41,500, and 41,500 $\mathrm{K}$ for CoStar, TLUSTY, WM-basic, and SNC02, respectively.

MCELS L 52 is ionized by M2002 19696 (Massey, 2002), an O6.5 V star (Figures 
4.1(f) and 4.8(b)). We find excellent agreement for lines with ionization potential (IP) below $40 \mathrm{eV}$, and all the atmosphere models produce consistent results. For [NeIII], whose IP $=40.96$, this agreement does not hold. The simulation using CoStar, the hardest atmosphere, over-predicts [NeIII], and TLUSTY under-predicts it. Interestingly, we find somewhat different nebular parameters produce the best fitting simulations between CoStar and the other atmosphere models. For CoStar, a slightly higher filling factor and lower density, 0.25 and $25 \mathrm{~cm}^{-3}$, respectively, work best. Whereas, $\epsilon=0.10$ and $n_{\mathrm{H}}=60 \mathrm{~cm}^{-3}$ produce better fits with the other atmosphere models. We note that this difference is close to our error in determining $\epsilon$ of 0.10 , and, thus, might not be significant. The best fitting $T_{\text {eff }}$ are $36,000,38,500,38,750$, and 38,500 K for CoStar, TLUSTY, WM-basic, and SNC02, respectively.

MCELS L 351 is ionized by OGLE-III 43846, which is also an O6.5 V star and, with a radius of $11 \mathrm{pc}$, it is the largest nebula in our sample (Figures 4.1(b) and 4.8(c)). We detect [OIII] $\lambda 4363$ in this object, from which we calculate $T_{e}$ and $\log (\mathrm{O} / \mathrm{H})($ Table 4.2). However, our [OIII] $\lambda 4363$ detection is at a very poor signalto-noise, and our result is consistent with the mean LMC metallicity. Therefore, we use the mean LMC metallicity in the CLOUDY simulations. As with MCELS L 28, the TLUSTY model atmosphere with $\epsilon=1$ is able to reproduce the [OIII]/[OII] ratio, although for that simulation the other emission lines are not well predicted. For the $\epsilon=0.1$ simulations, we find excellent agreement between simulations and observations for emission lines with IP $<40 \mathrm{eV}$. We note that the $Q_{0, \mathrm{SED}} / Q_{0, \mathrm{H} \alpha}$ comparison (Table 4.4) shows that $Q_{0, \mathrm{SED}}$ is less than half that of $Q_{0, \mathrm{H} \alpha}$, which is suggestive of a binary. This is supported by the fact that the observed $M_{V}$ is brighter than that for other stars of similar spectral type. However, the rectified stellar spectrum does not show features of multiple spectral types, which indicates that if this nebula is ionized by a binary system, it most likely an equal mass binary. In this case, the shapes of the SEDs will be similar, and, to a first order approximation, we can treat the nebula as a single star system. The best fitting $T_{\text {eff }}$ are $36,500,39,500,39,000$, and $39,000 \mathrm{~K}$ for CoStar, TLUSTY, WM-basic, and SNC02, respectively.

DEM L 283b is ionized by OGLE-III 44989, an O6.5 V((f)) star (Figures 4.1(c) 
and $4.8(\mathrm{~d})$ ). Since we do not have IMACS photometry for this object, we use the OGLE-III $V$-band magnitude to calculate the stellar luminosity. From the [OIII] $\lambda 5007$ line profile, we assign $R_{\text {inner }}=0.25 R_{\text {nebula }}$. Overall, we find good fits for all atmosphere models for lines with IP $<40 \mathrm{eV}$ and $\epsilon=0.10$. The best fitting $T_{\text {eff }}$ are 36,500, 39,000, 39,000, and 39,000 K for CoStar, TLUSTY, WM-basic, and SNC02, respectively.

MCELS L 344 is ionized by OGLE-III 45830, an O6.5-7 V((f)) star (Figures 4.1(d) and 4.9(a)). We note that the nebular structure diverges from a Strömgren sphere to the east in Figure 4.1(d). However, our slit position runs N-S and covers the regular portion of the nebula. Overall we find excellent fits for models where IP $<40 \mathrm{eV}$. The measured [NeIII] flux shows a $45 \%$ difference between the spectra from 2008 January 29 and 31, which results in a large scatter for [NeIII]/H $\beta$ in Figure 4.9(a). This apparent discrepancy is caused by the large observational uncertainty. Interestingly, for January 31, the simulation using CoStar is the only one to match the observations, and the others under-predict [NeIII] by $>50 \%$. The best fitting $T_{\text {eff }}$ are 37,000, 39,500, 39,000, and 39,000 K for CoStar, TLUSTY, WM-basic, and SNC02, respectively.

MCELS L 43 is ionized by M2002 17251, an O8 V star (Figures 4.1(e) and 4.9(b)). As noted in Section 4.3.1, MCELS L 43 is one of the few objects for which [OIII]/[OII] is well predicted by simulations using $\epsilon=1$ with atmosphere models from WMbasic, SNC02 and CoStar. However, the right panel of Figure 4.6 shows that these models cannot simultaneously match the $\mathrm{HeI} / \mathrm{H} \beta$ line ratios, which are a more reliable $T_{\text {eff }}$ indicator. At the temperatures required to match HeI intensity, [OIII] $\lambda 5007$ is over predicted by a factor of $2-3$. The fit improves in all emission lines when we decrease the filling factor to 0.10 (Figure 4.9(b)). The best fitting $T_{\text {eff }}$ are 37,000, 39,000, 39,000, and 39,000 K for CoStar, TLUSTY, WM-basic, and SNC02, respectively.

MCELS L 390 is ionized by OGLE-III 28307, an O9 V star (Figures 4.1(g) and $4.9(\mathrm{c}))$. For this object, the simulation using CoStar reproduces the relative amounts of [OIII] and [NeIII], while the others do not. This is in contrast to the rest 
of our sample, for which the CoStar models are too hard. This star is an eclipsing binary, according to the OGLE-III catalog of eclipsing binaries (Graczyk et al., 2011). However, the second star only contributes $10 \%$ of the $I$ band flux. Furthermore, Table 4.4 shows agreement between $Q_{0, \mathrm{SED}}$ and $Q_{0, \mathrm{H} \alpha}$. Therefore, the second star is not significant. The best fitting $T_{\text {eff }}$ are $35,000,35,000,37,000$, and 37,000 $\mathrm{K}$ for CoStar, TLUSTY, WM-basic, and SNC02, respectively.

MCELS L 394 is ionized by OGLE-III 28239, a star whose spectral type is between an $\mathrm{O} 9$ and a B1.5 V, based on the noisy stellar spectrum (Figures 4.1(g) and $4.9(\mathrm{~d})$ ). However, we assign this star the earlier $\mathrm{SpT}$ in this range because the observed [NeIII] rules out stars with SpT of B0 V or later. As with MCELS L 28, the oxygen lines are well fitted by TLUSTY simulations with $\epsilon=1$, but the other emission lines are not adequately reproduced in those simulations. Even with $\epsilon=$ 0.10 , it is still challenging to reproduce the $[\mathrm{SII}] / \mathrm{H} \beta,[\mathrm{OII}] / \mathrm{H} \beta$ and $[\mathrm{NII}] / \mathrm{H} \beta$ line ratios. As seen in Figure 4.9(d) the predictions lie at the edge of our $20 \%$ tolerance limit. Additionally, all the atmosphere models, except for CoStar, do not produce enough flux at the energies needed to reproduce the observed [NeIII]/H $\beta$ emission-line ratio (Figure 4.9(d)). The best fitting $T_{\text {eff }}$ are 37,000, 39,000, 39,000, and 39,000 $\mathrm{K}$ for CoStar, TLUSTY, WM-basic, and SNC02, respectively.

Unlike the other objects, $Q_{0, \mathrm{H} \alpha}$ in MCELS L 394 is a factor of a few less than $Q_{0, \text { SED }}$ (Table 4.4). This could indicate that the nebula is optically thin. If the nebula were optically thin, we expect to observe less [OII] and [SII] than if the nebula were optically thick. Based on our modeling, however, we see the opposite. We observe too much [OII] and [SII] relative to the optically thick model. Therefore, while the $\mathrm{H} \alpha$ photometry may suggest an optically thin nebula, it is clear that this does not adequately explain all the observations.

MCELS L 32 is ionized by OGLE-III 8229, a B0 V star (Figure 4.1(a) and 4.10(a)). [NeIII] is not detected in this nebula, and thus provides a firm upper limit for the $T_{\text {eff }}$ of the ionizing star. Both of the grids generated with the WM-basic code include atmosphere models down to $T_{\text {eff }}=30,000 \mathrm{~K}$, which is adequate for this $\mathrm{B} 0$ star. However, the CoStar grid in CLOUDY does not have dwarf B star models. The 
coolest CoStar dwarf, $T_{\text {eff }}=34,000 \mathrm{~K}$, is still a bit too hard to match the observed emission lines. We optimized the $T_{\text {eff }}$ to reproduce the $\mathrm{HeI} / \mathrm{H} \beta$ line ratios, with the result that $[\mathrm{OIII}] / \mathrm{H} \beta$ is under-predicted. The best fitting $T_{\text {eff }}$ are $34,000,33,000$, 34,000, and 34,000 K for CoStar, TLUSTY, WM-basic, and SNC02, respectively.

MCELS L 35 is ionized by OGLE-III 8203, one of our two supergiants, a B0 Ib star (Figures 4.1(a) and 4.10(b)). In addition to varying the $T_{\text {eff }}$ and $\epsilon$, we vary the surface gravity between $\log (g)=2.5--3.5$, as appropriate for supergiants (Crowther et al., 2006; Trundle et al., 2004). TLUSTY is the only atmosphere code which includes this whole range of gravities and temperatures. The lowest gravity in the available grids for WM-basic and SNC02 are 3.0 and 2.95, respectively. In the case of CoStar, the lowest available gravity for $T_{\text {eff }}>26,000 \mathrm{~K}$ is $\log (g)=2.86$. For TLUSTY, we find that simulations using $\log (g)=2.6-3.0$ all yield good fits, although at different temperatures. For example, for $\log (g)=2.6$, the best fitting $T_{\text {eff }}=27,250$ $\mathrm{K}$, whereas for $\log (g)=3.0, T_{\text {eff }}=28,500 \mathrm{~K}$. For WM-basic, SNC02 and CoStar we use $\log (g)=3.5$ and find $T_{\text {eff }}=31,000 \mathrm{~K}$ for all three atmospheres.

MCELS L 345 is ionized by OGLE-III 50093, the other supergiant in our sample, a B0.5 Iab star (Figures 4.1(d) and 4.10(c)). Based on the [NII]/[OII] ratio, this object has a much higher nitrogen abundance than the rest of our sample, $\log (\mathrm{N} / \mathrm{O})=-0.77$ compared to the mean LMC value of $\log (\mathrm{N} / \mathrm{O})=-1.5$. This higher abundance is confirmed by the simulations because those that use the mean value are unable to reproduce the $[\mathrm{NII}] /[\mathrm{OII}]$ line ratio. [OIII] $\lambda 5007$ is not detected in this object, which places an upper limit of $T_{\text {eff }}=26,000 \mathrm{~K}$. As discussed for MCELS L 32, we are only able to test the TLUSTY atmosphere models for this object. Note, the points along the bottom of Figure 4.10(c) are lines for which we only have upper limits on the line strength, and the simulations are consistent with the data. We find the best fitting $T_{\text {eff }}$ falls in the range of $22,000-24,000 \mathrm{~K}$.

MCELS L 346 is ionized by an eclipsing binary, MACHO 81.9725.16 (Alcock et al., 1997). The spectral type determined from our observed data is B1-1.5 V (Figure 4.2). However, we do detect [NeIII] in this object, despite the late SpT. The strengths of the [NeIII] and [OIII] lines suggest that this nebula is ionized by a star of much 
earlier spectral type. Based on the $[\mathrm{OIII}] / \mathrm{H} \beta$ and $[\mathrm{NeIII}] / \mathrm{H} \beta$ line ratios compared to the rest of our sample, the ionizing star in this nebula likely has an SpT between $\mathrm{O} 8$ $\mathrm{V}$ and $09.5 \mathrm{~V}$. This analysis is further supported by the measured $\log \left(Q_{0, \mathrm{H} \alpha}\right)=48.48$, which falls into the [47.99-48.65] range spanned by the O8-O9 V stars in our sample. Considering the short period of this binary, 1.2 days, it is possible that we observed the nebula when the earlier type star was eclipsed by a later type companion. Since the early type star will dominate the ionizing SED, we model this object with a single atmosphere model.

We note that one side of this nebula has an irregular morphology, while the other is more Strömgren-like (Figures 4.1(h)). Therefore, we only use the emission-line ratios from the portion of the slit that lies along the regular half of the nebula. The best fitting models are shown in Figure 4.10(d). These models have $R_{\text {inner }}=0.25 R_{\text {nebula }}$, $\epsilon=0.10$, and $n_{\mathrm{H}}=100 \mathrm{~cm}^{-3}$. We find excellent fits for WM-basic, TLUSTY, and SNC02 except at [NeIII] $\lambda 3869$, while CoStar over-predicts both [OIII] and [NeIII]. The best fitting $T_{\text {eff }}$ are 36,000, 37,500,38,000, and 37,000 $\mathrm{K}$ for CoStar, TLUSTY, WM-basic, and SNC02, respectively.

\subsection{Discussion}

\subsubsection{Comparison of Different Atmosphere Models}

In the previous section we discussed the simulations for each object individually. However, the question at hand is: how well do the atmosphere models reproduce the ionizing population, in general? In this section, we discuss the trends we find across our sample. To look at the results collectively, we plot $F_{\text {pre }} / F_{\text {obs }}$ for individual emission lines as a function of the spectral type of the ionizing star. These plots are shown in Figure 4.12.

With the exception of [NeIII] $\lambda 3869$ (Figure 4.12(d)), HiI region simulations using CoStar are consistent with the other atmosphere models and the observations (Figure 4.12). For high ionization potential lines, such as [NeIII] shown in Figure 4.12(d), simulations with the CoStar atmospheres over-produce the line emission. Other stud- 
ies have similarly found that the CoStar SED is too hard at high energies (e.g., Oey et al., 2000; Morisset et al., 2004; Mokiem et al., 2004). Additionally, we find that a small range of $T_{\text {eff }}$ reproduces the observed nebular emission for most of our range in SpT (Figure 4.14). This implies that the SEDs generated by CoStar are less sensitive to changes in the atmospheres between stars of different SpT.

As with CoStar, the TLUSTY models reproduce most of the emission lines up to the ionization potential of $\mathrm{Ne}^{+2}$. At the IP of $\mathrm{Ne}^{+2}, 41 \mathrm{eV}$, the modeled HiI region spectrum under-predicts the line emission, and it does so to a greater degree than the other atmosphere models. This suggests that the TLUSTY atmosphere is not producing enough flux at high energies and reflects differences in the incident SEDs, as shown in Figure 4.13. Figure 4.13 compares the SEDs produced by different stellar atmosphere codes for two temperatures, $T_{\text {eff }}=35,000 \mathrm{~K}$ and $T_{\text {eff }}=41,000 \mathrm{~K}$. At both $T_{\text {eff }}$, the high energy slope of the TLUSTY SED is steeper than the other atmosphere models. Similarly, Simón-Díaz \& Stasińska (2008) show that for ionizing SEDs with $30,000 \leq T_{\text {eff }} \leq 40,000 \mathrm{~K}$, TLUSTY produces less flux at high energies than model atmospheres with expanding winds. Using IR emission lines, Morisset et al. (2004) also find that plane-parallel model atmospheres, TLUSTY included, are too soft between $27 \mathrm{eV}$ and $41 \mathrm{eV}$ to reproduce the observed nebular emission. This confirms that the treatment of the expanding wind plays an important role in reproducing the flux of ionizing photons with higher energies. Gabler et al. (1989) showed that selfconsistent treatment of the wind was particularly important for the continuum near the HeII ionizing edge at $54.4 \mathrm{eV}$. It was also shown by Sellmaier et al. (1996) that the [NeIII] problem, in which photoionization models consistently under-predicted [NeIII] $\lambda 3869$ (e.g., Simpson et al., 1995, 1986; Mathis et al., 1985; Rubin et al., 1991), is not an issue in simulations using non-LTE atmospheres that have a wind extension. We note that while there is a general trend for $[\mathrm{NeIII}] / \mathrm{H} \beta$ to be under predicted, the scatter is large and the degree to which $[\mathrm{NeIII}] / \mathrm{H} \beta$ is under predicted in our TLUSTY simulations is much less than that seen for earlier generations of stellar atmosphere models.

At the opposite energy regime, simulations using TLUSTY yield more [OII] emis- 
sion than the other atmosphere models, and typically over-predict [OII] $3727 \AA / \mathrm{H} \beta$ by 15\%-30\% (Figure 4.12(a)). While TLUSTY is softer (Simón-Díaz \& Stasińska, 2008), it is surprising that this trend is not seen in the other lines with similar ionization potential, such as [NII] $\lambda 6584$ (Figure 4.12(b)). One explanation for this could be the relative shapes of the SEDs above the ionization potentials of $\mathrm{N}^{+2}(29.6 \mathrm{eV})$ and $\mathrm{O}^{+2}$ $(35.1 \mathrm{eV})$, since the flux in that energy range controls the ionization from $\mathrm{O}^{+}$to $\mathrm{O}^{+2}$ and $\mathrm{N}^{+}$to $\mathrm{N}^{+2}$. For energies greater than $35 \mathrm{eV}$, the luminosity of the TLUSTY SED drops relative to other models, at least for $T_{\text {eff }}<40,000$ K (Simón-Díaz \& Stasińska, 2008). Thus, the slight excess of [OII] may result from the softer SED.

Simulations using atmospheres generated with the WM-basic code are the closest to the observed values for most emission lines (Figure 4.12a-c), and thus best represent the ionizing stars. However, Figure 4.12(d) shows that the predictions for [NeIII] $\lambda 3869 / \mathrm{H} \beta$ have more scatter than what is seen for the emission lines of lower IP. The ratio of predicted and observed flux for $[\mathrm{NeIII}] / \mathrm{H} \beta$ ranges from 0.7 to 1.7, with most points under-predicted by $20 \%-30 \%$. As expected, the atmosphere models

presented in the SNC02 grid are consistent with the WM-basic grid implemented in CLOUDY, although we find that they occasionally require a slightly different $T_{\text {eff }}$. This is likely due to the different stellar properties (stellar radius, mass loss rate and wind terminal velocity) used in generating the atmosphere model grid, as discussed in Section 4.1.1. We note that the temperature differences, when they occur, are in the range of 250-750 K. Although the predictions from the two grids are close, the WM-basic grid does slightly better than the $\mathrm{SNC02}$ grid in reproducing [OIII]/H $\beta$ for late SpT stars.

\subsubsection{Calibration between $\mathrm{SpT}$ and $T_{\text {eff }}$}

The calibration between spectral type and effective temperature is an important consequence of the atmosphere modeling. Ordinarily, the calibration is based on careful fitting of photospheric absorption lines in model SEDs to reproduce highresolution stellar spectra. Therefore, the $\mathrm{SpT}-T_{\text {eff }}$ calibration depends on the stellar atmosphere models used in the fitting. This bias is especially apparent when one 
compares the calibrations based on atmospheres codes without line blanketing (e.g., Vacca et al., 1996) to calibrations based on atmospheres with line blanketing (e.g., Martins et al., 2005; Massey et al., 2005). The latter calibrations assign effective temperatures that are several thousand degrees cooler than the previous calibrations.

In contrast to this method, the effective temperatures from Table 4.3 depend on reproducing the ionization balance in the nebula that the star ionizes. The points in Figure 4.14 show the effective temperatures that we generate as a function of the SpTs from Table 4.1. For comparison, the lines show calibrations from the literature for dwarf stars. The typical error on the literature calibrations is $\sim 1000 \mathrm{~K}$ (Martins et al., 2005). Figure 4.14 does not show MCELS L 346 since the SpT of the ionizing star is uncertain. MCELS L 394, for which the stellar spectrum is noisy, is placed at $\mathrm{SpT}=09.5$ with a large error bar. Based on the nebular spectrum, however, this object is probably at the upper end of the shown error bar.

We find that, in general, WM-basic, SNC02, and TLUSTY predict similar $T_{\text {eff }}$, while CoStar predicts cooler $T_{\text {eff }}$. This trend is consistent with our findings in Section 4.3.3; the CoStar SEDs are harder than the other atmosphere models, and therefore require a lower $T_{\text {eff }}$ to reproduce the observed emission-line spectra. For the earliest stars, the best fitting $T_{\text {eff }}$ selected for TLUSTY is slightly hotter than that of WM-basic and SNC02. At late SpT we see a trend toward the opposite. Since the treatment of the winds is the biggest difference between the TLUSTY and WM-basic atmospheres, this may reflect changes in the wind properties toward late SpT stars.

We note that the two supergiants in our sample have $T_{\text {eff }}$ between $24 \mathrm{kK}$ and 31 $\mathrm{kK}$. This large range spans, and is consistent with, the expected $T_{\text {eff }}$ for Magellanic class I stars 25-32 kK (e.g., Mokiem et al., 2007; Trundle et al., 2004).

The effective temperatures presented in Figure 4.14 are consistent with the relations in the literature, within the errors. However, we note that the points appear to follow a shallower slope. This is primarily caused by the stars of later spectral type. The O5.5 - O7 V stars follow the LMC calibration of Massey et al. (2005, ; thick-dashed line), but the later spectral types prefer the hotter temperatures of the Vacca et al. (1996) calibration (dotted line). 
One contributing factor to the $T_{\text {eff }}$ trend is the assumed $\log (g)$. We calculate an expected variation in $\log (g)$ for dwarf stars across our spectral range of 0.05 dex, based on the modeled stellar parameters from Schaerer et al. (1993). If there is an error in our spectroscopically determined luminosity class, it will affect the best fitting $T_{\text {eff }}$ selected. Specifically, if any of the stars classified as dwarfs are actually giants or supergiants, the $T_{\text {eff }}$ will be 1000-3000 K cooler than the $T_{\text {eff }}$ chosen for a dwarf star. The difference between the $\log (g)$ appropriate for dwarfs and supergiants increases for later SpT. Therefore, changes to the SED caused by uncertainty in $\log (g)$ will be strongest for stars of late spectral type (e.g., Martins et al., 2005). We find 0-1000 $\mathrm{K}$ decrease in $T_{\text {eff }}$ for MCELS L 28, an O5.5 V, going from $\log (g)=4.0$ to $\log (g)=3.0$, but a 2000-3000 K decrease in $T_{\text {eff }}$ for MCELS L 20, an O8 V.

Another consideration is the metallicity for which these $\mathrm{SpT}-T_{\text {eff }}$ calibrations are valid, since lower metallicity stars will have higher $T_{\text {eff }}$ (Massey et al., 2005; Mokiem et al., 2004). All of the literature calibrations, except for the thick-dashed line, are calibrations that use solar metallicity stars. Thus, we expect most of these calibrations to lie at cooler $T_{\text {eff }}$ than the points in Figure 4.14. While this is the case for the calibrations based on line blanketed models, which we see by comparing the thickdashed line to the thin-dashed and solid lines, we note the scatter between the points is larger than the difference in $T_{\text {eff }}$ between the LMC and the Milky Way calibrations. Furthermore, the scatter is comparable to the difference between calibrations based on atmospheres with and without line blanketing.

The conventional approach to obtaining the SpT- $T_{\text {eff }}$ calibration is based on photospheric lines and is sensitive to the SED at the photosphere. Our method, however, depends on the ionization balance of the nebula and is also sensitive to changes in the SED due to layers outside the photosphere. Thus, SpT $-T_{\text {eff }}$ relations derived from nebular data have the potential to probe factors in the upper layers of the atmosphere models that do not match the conditions in the ionizing stars. Our sample is small, and as seen earlier, there are a variety of uncertainties, limiting its usefulness in this regard, but with larger samples and better data, this approach promises an alternative and important way to refine SpT- $T_{\text {eff }}$ calibrations. 


\subsection{Conclusions}

Diagnostics from Hiı region emission-line spectra are used extensively to determine the physical conditions of the ISM of galaxies, as well as understand their stellar populations and their chemical evolution. The shape of the ionizing SED is one of the primary properties that determines the HII region emission-line spectrum. However, the intrinsic shapes of massive star SEDs are uncertain, and we depend on atmosphere models to describe them. It is therefore crucial to understand and quantify how well massive star atmosphere models represent the ionizing sources.

In this study, we compare a sample of single-star HiI regions from the nearby LMC to photoionization simulations ionized by atmosphere models from widely-available CoStar, TLUSTY, and WM-basic atmosphere grids. We select our sample of small, Strömgren sphere-like HII regions from narrowband MCELS images. We obtain spectra and photometry of this sample using IMACS on the Magellan Baade Telescope at Las Campanas Observatory. We assign spectral types to the ionizing stars in our sample and find a range from $\mathrm{O} 5.5 \mathrm{~V}$ to B0 V with 10 of our $12 \mathrm{HII}$ regions ionized by dwarf stars. While we choose Hiı regions that are likely to be ionized by a single star, contamination by OB companions cannot be entirely ruled out. Two of the objects in our sample, MCELS L 346 and MCELS L 390, are confirmed eclipsing binaries. MCELS L 351 may contain a nearly equal mass binary, based on comparing $Q_{0, \mathrm{SED}}$ and $Q_{0, \mathrm{H} \alpha}$. The rest of our sample show no obvious signs of OB companions, such as composite spectral types or large discrepancies between $Q_{0, \mathrm{H} \alpha}$ and $Q_{0, \mathrm{SED}}$, and as discussed in Section 4.2.2 the binary status will not affect our results.

We evaluate the stellar atmosphere models by matching the emission-line spectra predicted by CLOUDY photoionization simulations with that observed in the nebulae. Our nebular simulations show that uniformly filled Strömgren spheres cannot reproduce the observations. In these cases, $[\mathrm{SII}] / \mathrm{H} \beta,[\mathrm{OII}] / \mathrm{H} \beta$ and $[\mathrm{NII}] / \mathrm{H} \beta$ are under-predicted, which suggests that the ionization parameter is too high in these simulations. We explore the effects of changing $R_{\text {inner }}$ and $Z$, but find that neither parameter can resolve this issue. Additionally, we find that $[\mathrm{SII}] / \mathrm{H} \beta$ and $[\mathrm{OI}] / \mathrm{H} \beta$ 
depend strongly on the nebular parameters, but only weakly on the particular SED used in the simulation. In contrast to the uniformly filled Strömgren spheres, simulations that assume a clumpy medium reproduce the observed data. The clumpy medium is described by specifying a filling factor of dense gas and assuming that the remaining volume is a vacuum. In general, we find that a filling factor of $\sim 0.10$ produces simulations that agree with observations.

We compare the rate of ionizing photons predicted by the best fitting atmosphere, assuming a constant $\log (g)$, with the rate calculated from $L(\mathrm{H} \alpha)$. Within the errors due to uncertainty in $\log (g)$, which can be as high as a factor of two, we find that the two values are consistent. However, we do find a systematic offset between the rate of ionizing photons predicted by different atmosphere models. TLUSTY produces the highest $Q_{0}$, while WM-basic and SNC02 have slightly lower rates. In contrast, the $Q_{0}$ from CoStar is a factor of two lower than the other atmosphere models. The hardness of the CoStar SED can explain this offset. We select the best-fitting atmosphere models by matching the ionization balance in the nebula. Since the CoStar models have harder SEDs, the best-fitting CoStar atmospheres are a few thousand degrees cooler and produce fewer ionizing photons as a result.

We evaluate the stellar atmosphere models by comparing the predicted to observed emission line ratios. For emission lines with IP $\leq 36 \mathrm{eV}$, we find that simulations ionized by WM-basic and SNC02 atmospheres, reproduce the observations. Simulations using TLUSTY are also consistent, with the exception of [OII] $\lambda 3727$. For this line, TLUSTY over-predicts the amount of $[\mathrm{OII}] \lambda 3727 / \mathrm{H} \beta$ in the nebula. Surprisingly, despite having the most approximate treatment of line blanketing, simulations using CoStar reproduce the emission lines up to [OIII], at $35.1 \mathrm{eV}$, but they do so at lower $T_{\text {eff }}$ than the other model atmospheres.

At energies $>36 \mathrm{eV}$, the predictions from different atmosphere models diverge from each other and the observations. We find significant scatter between the predictions and observations of $[\mathrm{NeIII}] \lambda 3869 / \mathrm{H} \beta$ from all atmosphere models. CoStar, the hardest atmosphere over-predicts the $[\mathrm{NeIII}] / \mathrm{H} \beta$ line ratio by more than a factor of two in most of our objects. In contrast, the other atmosphere models range from 
over- to under-predicting $[\mathrm{NeII}] / \mathrm{H} \beta$ by $70 \%$. Simulations ionized by TLUSTY, with its softer SED and plane-parallel geometry, systematically produce less $[\mathrm{NeIII}] / \mathrm{H} \beta$ than simulations using WM-basic and SNC02.

Finally, we compare the SpT- $T_{\text {eff }}$ calibrations in the literature with the best fitting $T_{\text {eff }}$ from the simulations in this study. Our results for stars with SpT earlier than O8 V fall along the LMC calibration of Massey et al. (2005), while stars later than O8 V seem to be hotter than the calibrations predict. The SpT- $T_{\text {eff }}$ calibrations from the literature are based on photospheric lines, while the effective temperatures obtained from this work depend the ionization balance of the nebulae. The comparison of these two methods has the potential to reflect differences between the outer atmospheres of the models and the actual ionizing stars. However, there are a variety of uncertainties and our sample is small, which limits its usefulness in this regard. Further study with a larger sample and detailed stellar modeling is needed to determine if the deviations at late $\mathrm{SpT}$ are significant.

This work uses single-star HII regions to test the predictions of stellar atmosphere models. By using single-star Strömgren spheres, we dramatically reduce the free parameters involved in evaluating atmosphere models with observed nebulae. Future directions that could improve on the groundwork presented here include leveraging a larger range in $T_{\text {eff }}$ by including stars earlier than $05.5 \mathrm{~V}$, using a detailed fit to high resolution stellar spectra to generate the input model SEDs, and evaluating model atmospheres at different metallicities. 


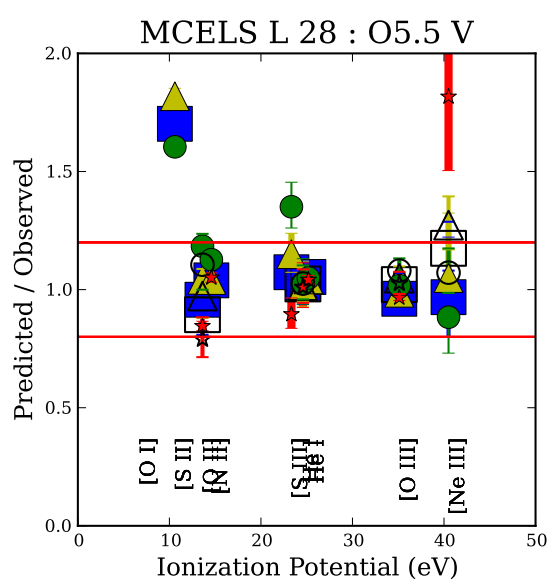

(a) MCELS L 28

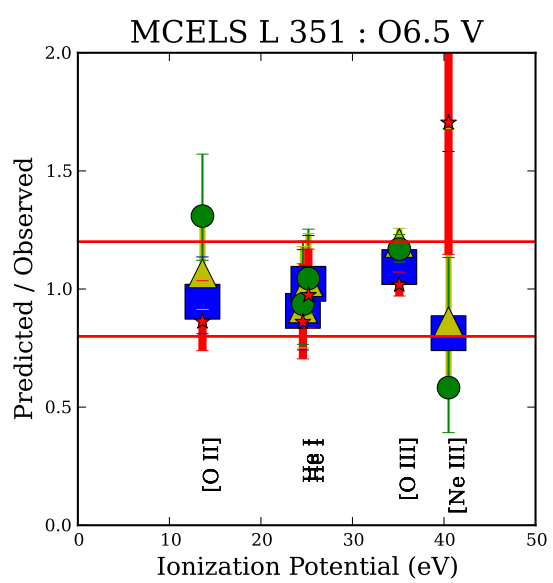

(c) MCELS L 351

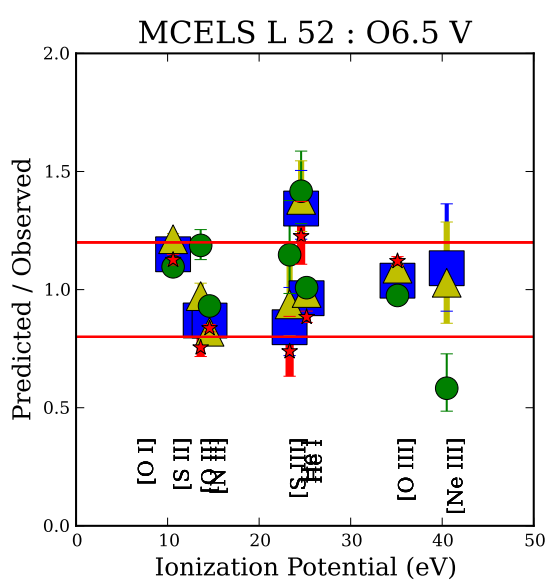

(b) MCELS L 52

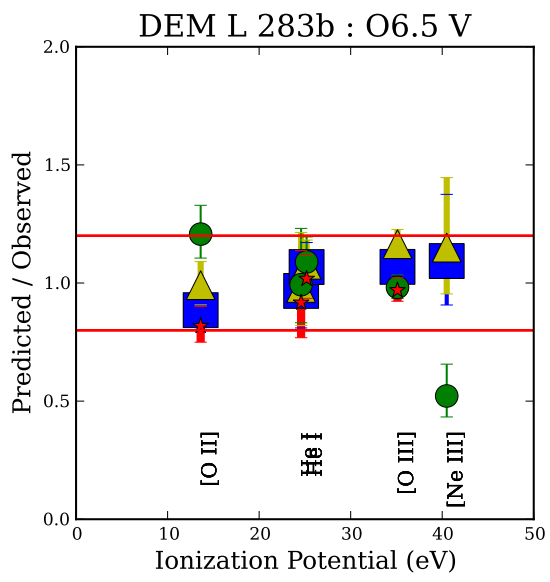

(d) DEM L 283b

Figure 4.8. The ratio of the predicted to observed emission-line intensities is plotted as a function of the ionization potential of the emission line for the simulation that has the closest match to our observations. The different symbols correspond to CLOUDY simulations that are ionized by different atmosphere models: CoStar, TLUSTY, WM-basic, and SNC02 are denoted by the red stars, green circles, blue squares, and yellow triangles, respectively. The filled data points use the observed flux from the night of 2008 January 30 or 31, while the hollow data points are from 2008 January 29. 


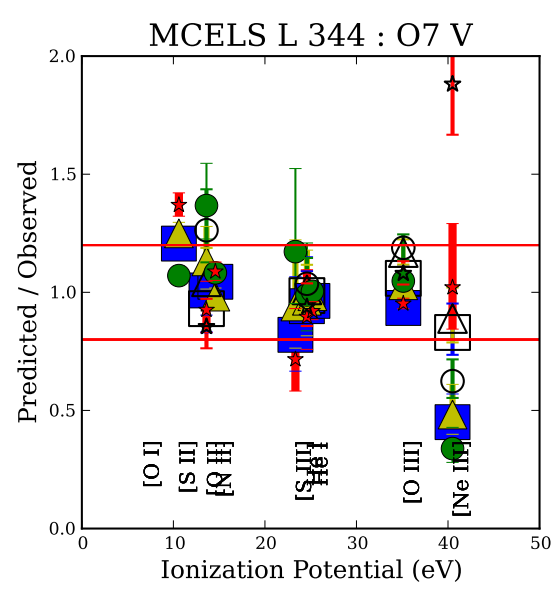

(a) MCELS L 344

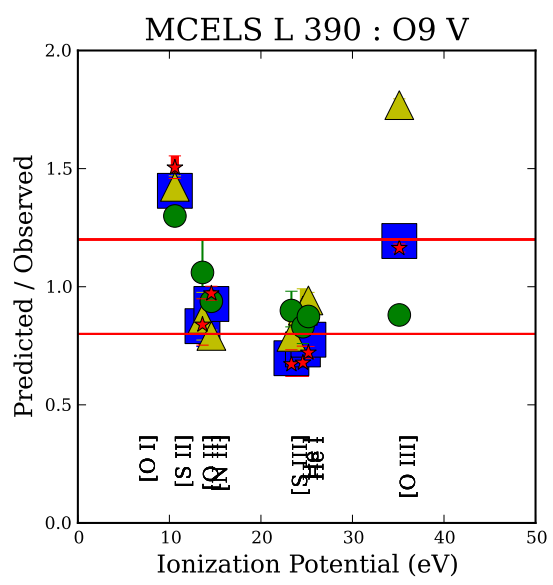

(c) MCELS L 390

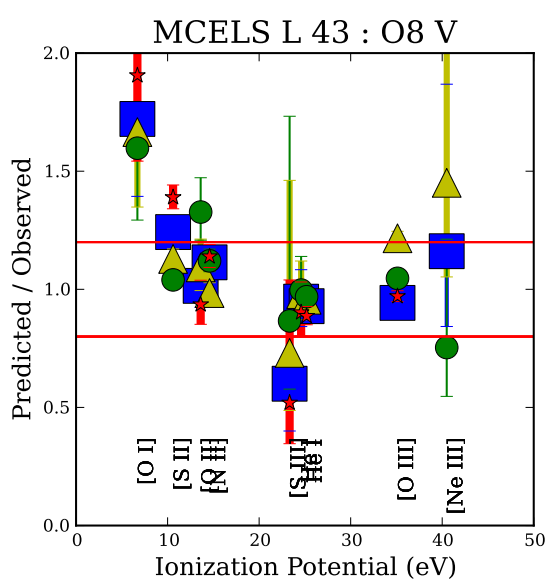

(b) MCELS L 43

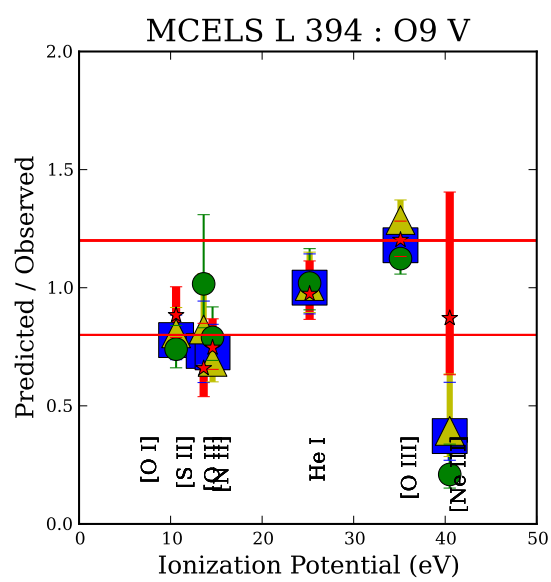

(d) MCELS L 394

Figure 4.9. Same as Figure 4.8, but for MCELS L 344, MCELS L 43, MCELS L 390, and MCELS L 394. The different symbols correspond to CLOUDY simulations that are ionized by different atmosphere models: CoStar, TLUSTY, WM-basic, and SNC02 are denoted by the red stars, green circles, blue squares, and yellow triangles, respectively. The filled data points use the observed flux from the night of 2008 January 30 or 31, while the hollow data points are from 2008 January 29. 


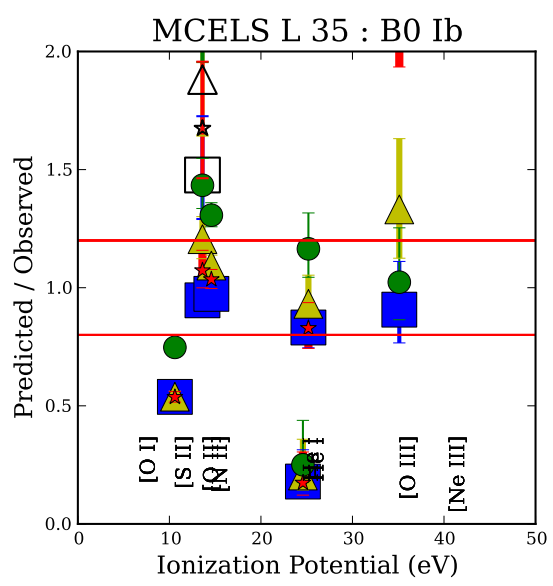

(a) MCELS L 35

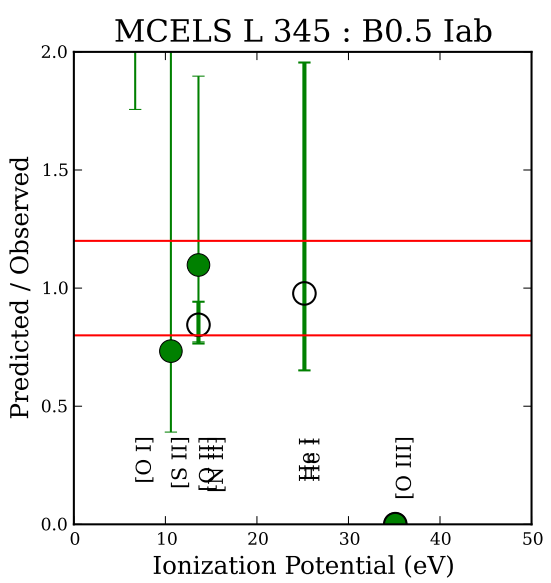

(c) MCELS L 345

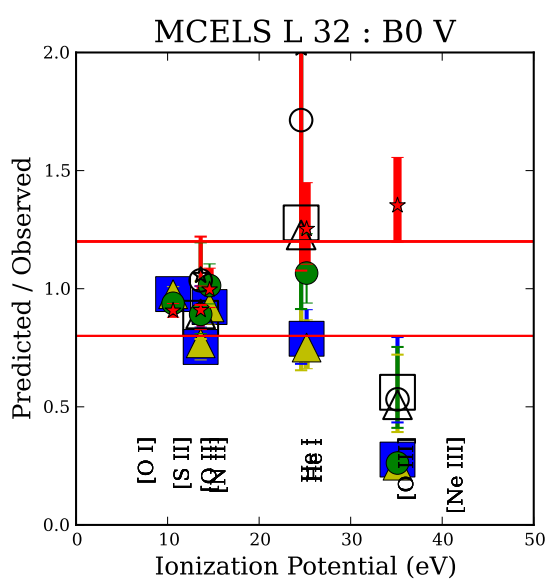

(b) MCELS L 32

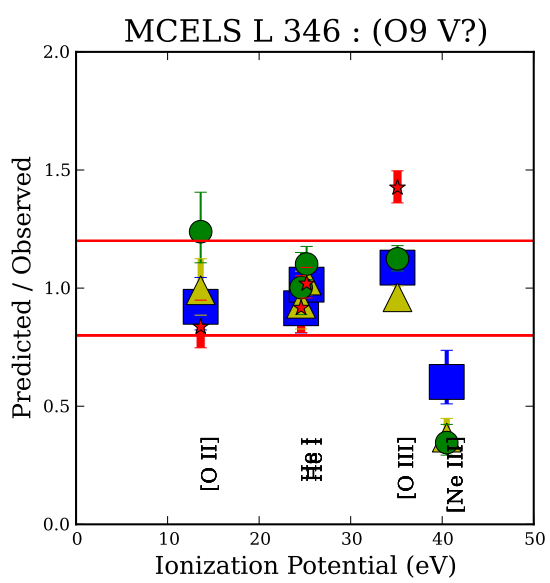

(d) MCELS L 346

Figure 4.10. Same as Figure 4.8, but for MCELS L 032, MCELS L 035, MCELS L 345, and MCELS L 346. 

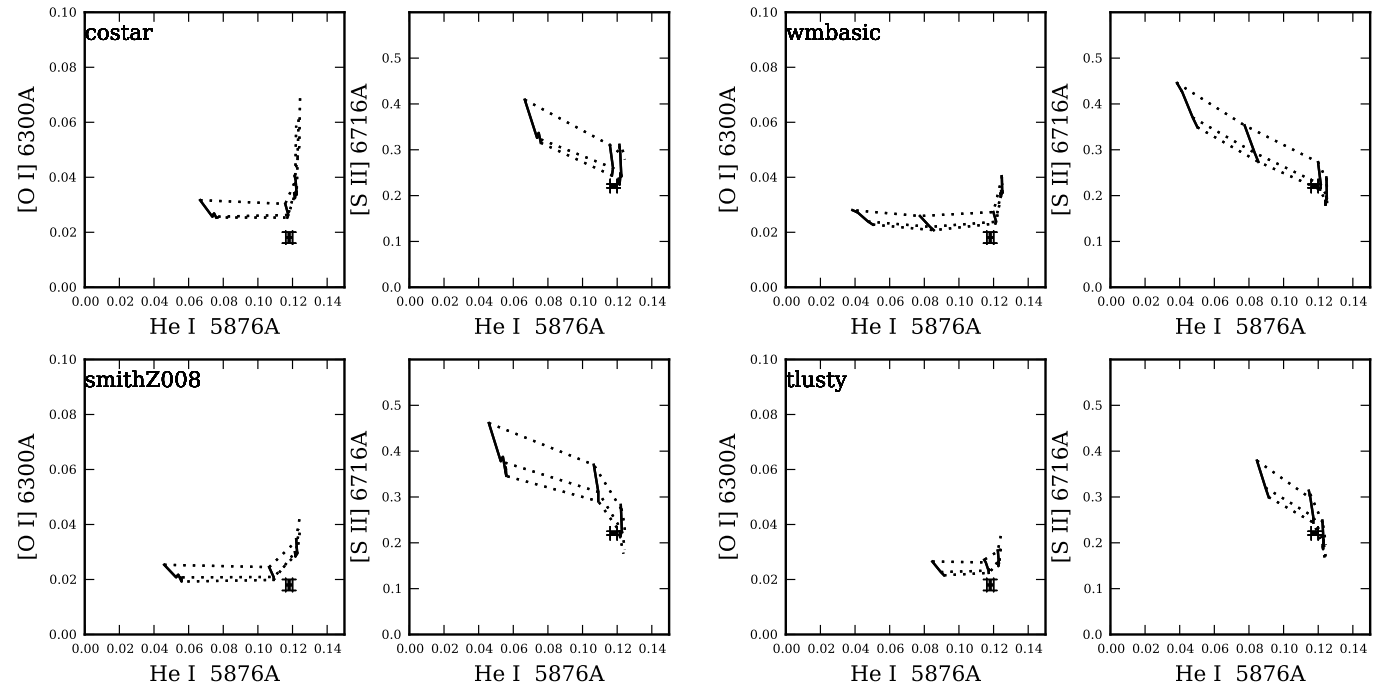

Figure 4.11. [OI] $\lambda 6300 / \mathrm{H} \beta$ and $[\mathrm{SII}] \lambda 6716 / \mathrm{H} \beta$ vs. HeI $\lambda 6876 / \mathrm{H} \beta$ for MCELS L 28. Dotted lines are models with constant $R_{\text {inner }}=0.10,0.25$, and $0.50 R_{\text {nebula }}$ and solid lines have constant $T_{\text {eff }}=35$, $37,39 \mathrm{kK}$, except WM-basic, which also includes $T_{\text {eff }}=40 \mathrm{kK}$. The values of $R_{\text {inner }}$ increase going upward and $T_{\text {eff }}$ increases from left to right. 


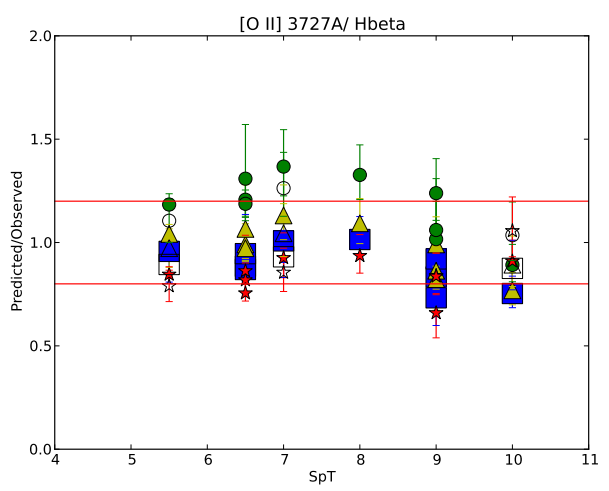

(a) [OII] $3727 \AA$

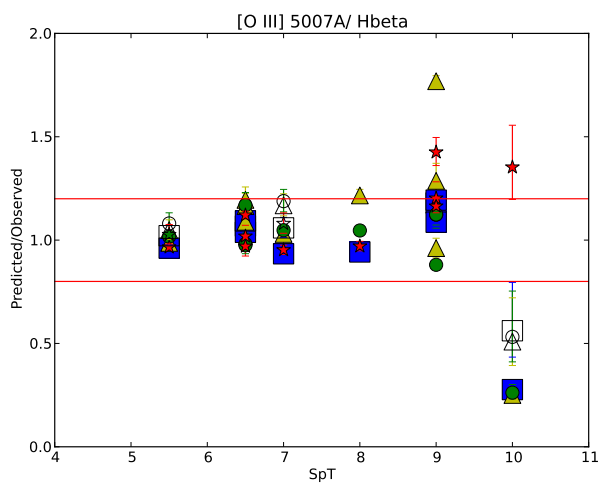

(c) $[$ OIII $] 5007 \AA$

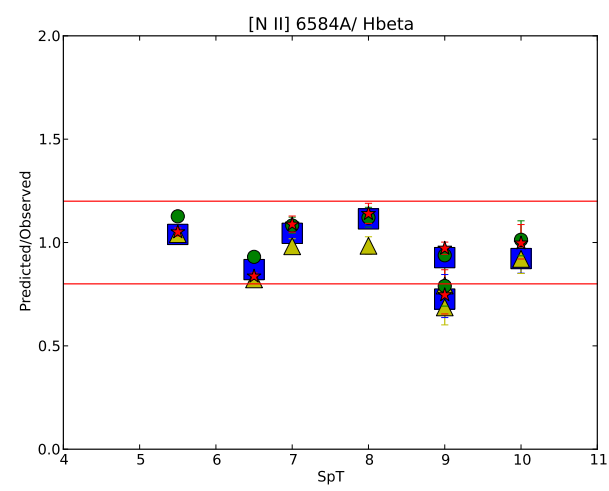

(b) $[\mathrm{NII}] 6584 \AA$

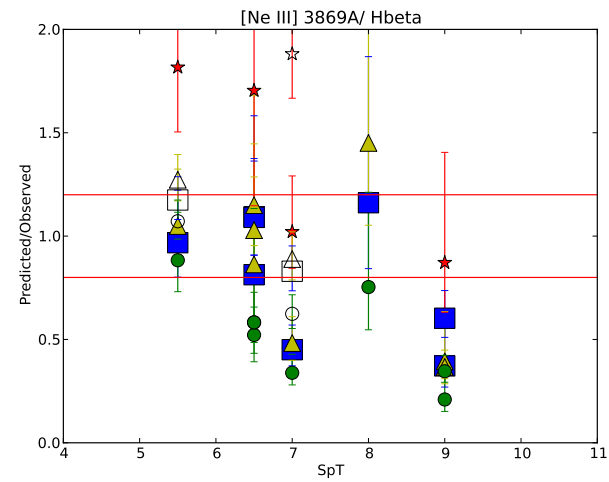

(d) $[\mathrm{NeIII}] 3869 \AA$

Figure 4.12. $F_{\text {pre }} / F_{\text {obs }}$ as a function of spectral type for [OII] $\lambda 3727,[\mathrm{NII}] \lambda 6584$, [OIII] $\lambda 5007$ and [NeIII] $\lambda 3869$ in panels $a, b, c$ and $d$, respectively. The different symbols correspond to CLOUDY simulations that are ionized by different atmosphere models: CoStar, TLUSTY, WM-basic, and SNC02 are denoted by the red stars, green circles, blue squares, and yellow triangles, respectively. The filled data points use the observed flux from the night of 2008 January 30 or 31, while the hollow data points are from 2008 January 29. Numbers $<10$ refer to $\mathrm{O}$ type stars, and numbers $\geq 10$ refer to B type stars, where 10 corresponds to B0. Colors and symbols are as for Figure 4.8. 


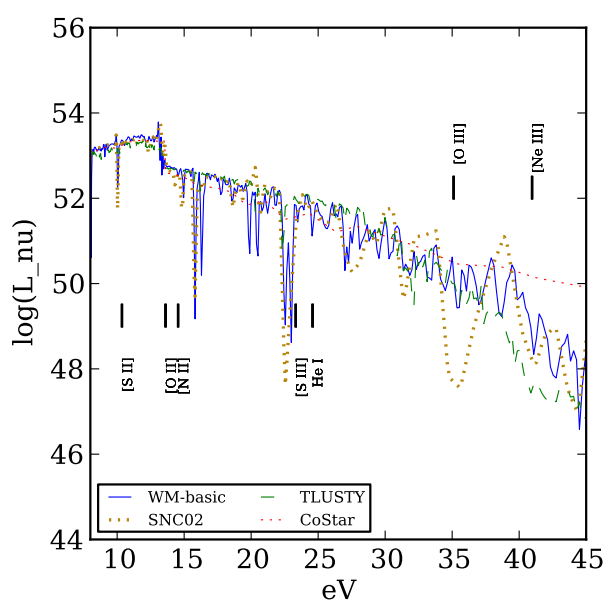

(a) $T_{\text {eff }}=41,000 \mathrm{~K}$

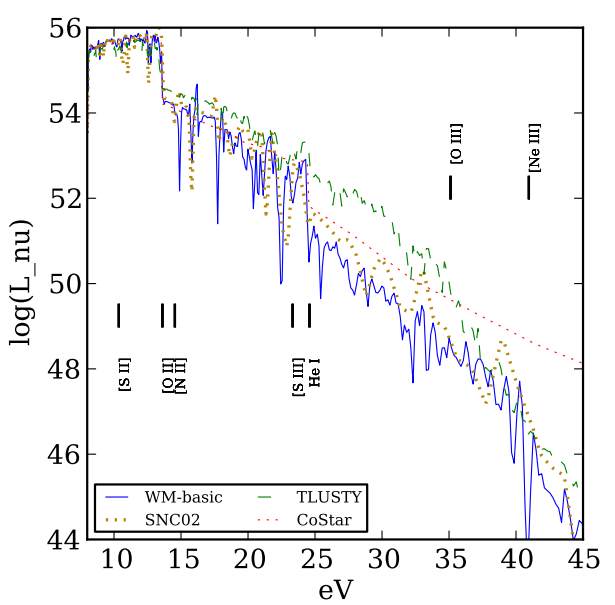

(b) $T_{\text {eff }}=35,000 \mathrm{~K}$

Figure 4.13. SEDs for stars with $T_{\text {eff }}=41,000 \mathrm{~K}$ and $\log \left(L_{\mathrm{bol}}\right)=37.09$ (top) and $T_{\text {eff }}=35,000 \mathrm{~K}$ and $\log \left(L_{\mathrm{bol}}\right)=37.78$ (bottom) for each stellar atmosphere model. The different colors correspond different atmosphere models: CoStar, TLUSTY, WM-basic, and SNC02 are denoted by the red, green, blue, and yellow lines, respectively. 


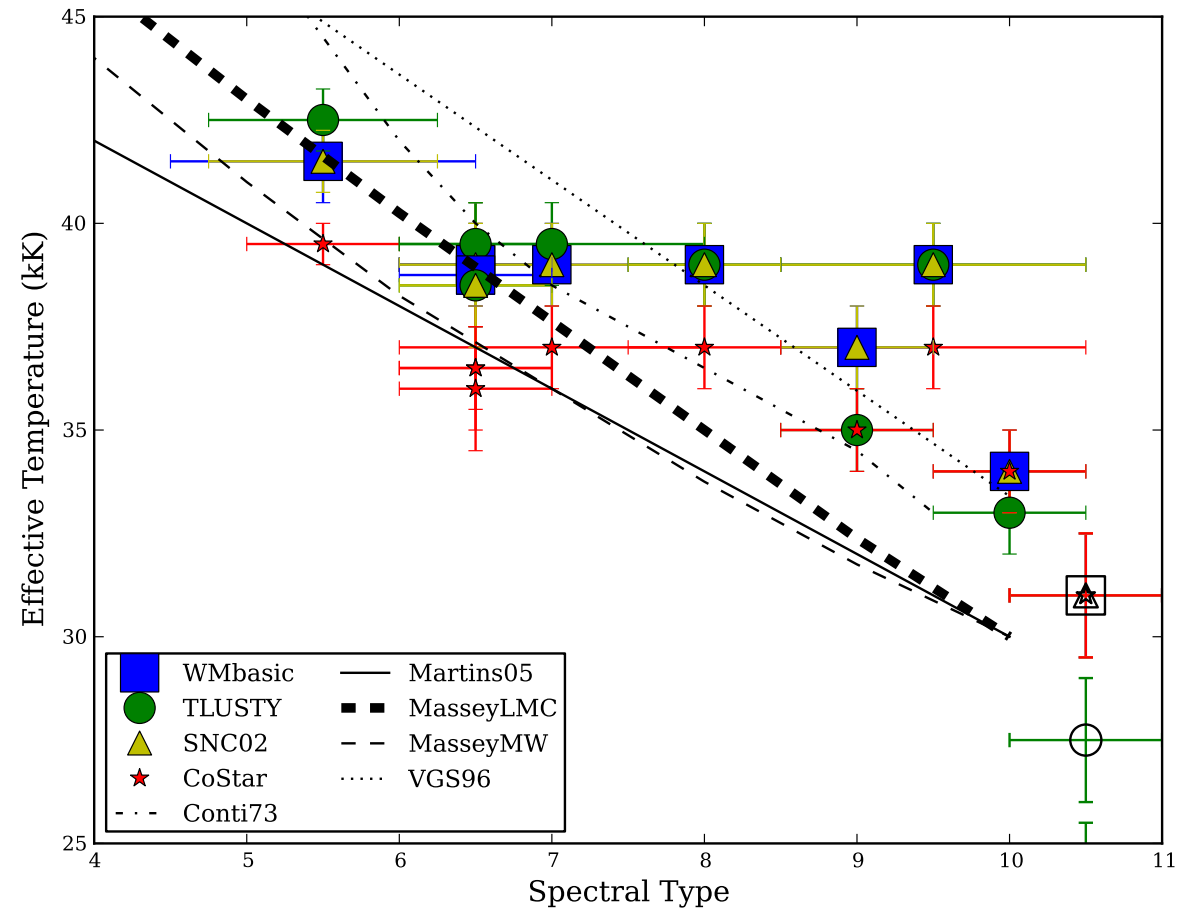

Figure 4.14. A comparison of the $T_{\text {eff }}$ from this paper to different $S p T-T_{\text {eff }}$ calibrations. Symbols represent temperatures derived from this work, while the lines are SpT- $T_{\text {eff }}$ calibrations for dwarf stars in the literature. Dash-dotted, solid, thin- and thick-dashed, and dotted correspond to calibrations from Conti (1973), Martins et al. (2005), Massey et al. (2005), and Vacca et al. (1996) respectively. We note that the thick dashed line is a calibration for the LMC while the rest are derived from Galactic stars. The hollow symbols correspond to the luminosity class I stars. The different symbols correspond to CLOUDY simulations that are ionized by different atmosphere models: CoStar, TLUSTY, WM-basic, and SNC02 are denoted by the red stars, green circles, blue squares, and yellow triangles, respectively. In this figure, numbers $<10$ refer to $\mathrm{O}$ type stars, and numbers $\geq 10$ refer to B type stars, where 10 corresponds to B0. 


\section{CHAPTER 5}

\section{Conclusions}

Ionizing radiation from massive stars plays a key role in our cosmic history, shaping the interstellar medium of galaxies, and emission-line based diagnostics. In this work we used spectra and narrowband images of photoionized gas to investigate the galactic properties that influence the escape of ionizing radiation from starbursts and to evaluate the spectral energy distributions (SED) predicted by stellar atmosphere models. We demonstrated two under-utilized methods for evaluating the effects of the ionizing radiation from massive stars: ionization parameter mapping (Pellegrini et al., 2012) and photoionization modeling of single-star HII regions. While our samples for the studies in this thesis were small, they still provided valuable insight on the ionizing radiation emitted by massive stars and its interaction with the interstellar medium (ISM).

In chapters 2 and 3, we investigated the processes and galaxy properties that influence the escape of ionizing radiation from the ISM of a galaxy. To accomplish this task, we used the technique of ionization parameter mapping (Pellegrini et al., 2012) to reveal the ionization structure in the extended ionized gas of seven nearby starbursts. For nebulae that absorb all the incident ionizing radiation, a transition from high to low ions is present at the interface of the nebula and the neutral environ-

ment. A lack of such transition indicates that ionizing radiation escapes beyond the observed nebulae (Pellegrini et al., 2012). [SIII] and [SII] narrowband images taken with the MMTF on the Baäde telescope at Las Campanas Observatory formed the basis for these maps.

From the ionization parameter maps, we discovered that NGC 5253 and NGC 3125 have optically thin ionization cones, which suggests that ionizing radiation may be 
escaping from these galaxies. The two ionization cones are quite narrow, with estimated opening angles of $40-70^{\circ}$. This morphology indicates that the passageways through which the ionizing photos travel are narrow as well. These results provide observational support for the 'picket-fence' method of escape and emphasizes the importance of the ISM morphology in controlling the fraction of ionizing radiation that escapes $\left(f_{\text {esc }}\right)$ (Fernandez \& Shull, 2011). If we assume axisymmetry, these opening angles imply solid angles of just $4-9 \%$ of $4 \pi$ steradians. Thus, the covering factor for $f_{\text {esc }}$ is much less than unity. Narrowband Lyman continuum studies of $z \sim 2-3$ starbursts show that when Lyman continuum is detected, it covers a smaller region on the sky than the non-ionizing UV continuum (e.g., Nestor et al., 2011). Not only is this consistent with the implied covering fractions in our sample, but it further links the ionization cones observed in NGC 5253 and NGC 3125 to the galaxies at high redshift that do have confirmed $f_{\text {esc }}$. If these ionization cones represent the general mode by which ionizing radiation escapes from starbursts, then an orientation bias can, at least partially, explain the low detection rate of escaping Lyman continuum.

In addition to depending on the orientation of a galaxy, our data suggest an optimal age for detecting escaping ionizing radiation. Both of the optically thin ionization cones are ionized by stellar populations with ages of 3-5 Myr. This age may represent the balance between maintaining a young population with a high $Q_{0}$ and the time it takes for bubbles to rupture and break out of the ISM. In contrast, NGC 1705, which shows evidence for being optically thick, is primarily ionized by older (10-15 Myr) stars. This older population may not generate enough ionizing photons to escape ISM of the galaxy. In addition, both galaxies have evidence for previous star forming episodes within the last 100 Myr. This suggests that prior episodes of star formation may have cleared passageways through which ionizing radiation from the current burst can escape. As modeled by Fujita et al. (2003), this scenario may result in a higher instantaneous $f_{\text {esc }}$. This young age supplements growing observational evidence in the literature for high $f_{\text {esc }}$ in galaxies with young stellar populations (e.g., Jaskot \& Oey, 2013; Bergvall et al., 2013).

Thus far, we have discussed the radiative transfer in starburst galaxies from a mor- 
phological perspective; where is the photoionized gas, and what is the morphology of the spatial changes to $[\mathrm{SIII}] /[\mathrm{SII}]$ ?. However, the shape of the ionizing spectral energy distribution (SED) is one key assumption that goes into both the interpretation of the ionization parameter maps and the calculations of $f_{\text {esc }}$ in Lyman continuum studies of starbursts. For example, if the ionizing is very soft, as it would be for a population that is dominated by B stars, then an ionized region may be optically thin and still exhibit a transition zone in ionization parameter maps (Pellegrini et al., 2012). Furthermore, the calculation of $f_{\text {esc }}$ from intermediate redshift galaxies includes the ratio of ionizing to non-ionizing UV emission (e.g., Siana et al., 2007; Iwata et al., 2009). This fraction will depend on the assumed properties of the ionizing population and the shape of the ionizing SED. However, the shapes of the SEDs of massive stars are uncertain, and we depend on stellar atmosphere models to describe them. These models use different algorithms to incorporate complicated stellar physics, such as non-LTE conditions, line blocking and blanketing, and wind blanketing. An ongoing, critical issue is understanding which of these model atmospheres best represent the shape of the massive-star ionizing SED (e.g., Simón-Díaz \& Stasińska, 2008; Oey et al., 2000; Crowther et al., 1999; Esteban et al., 1993).

In chapter 4, we addressed this issue using a sample of single-star Hı regions in the LMC. By comparing the predicted emission-line spectra from tailored CLOUDY photoionization simulations to the observed spectra, we determined which model atmosphere best represented the ionizing stars in our sample. We found that uniformly filled Strömgren spheres cannot reproduce the observations. These simulations consistently under-predicted [SII], [OII], and [NII], which suggests that the simulated ionization parameter is too high. Neither changing the inner radii nor the metallicity resolved the discrepancy. Instead, we found that Strömgren spheres with a clumpy gas distribution, described by a filling factor, $\epsilon \sim 0.1$, reproduced both high and low ions simultaneously. In addition, we found that [SII] and [OI] depend strongly on the nebular parameters and weakly on the particular SED. This behavior indicates that these lines are better diagnostics for the nebular environment than the ionizing star.

In general, the SEDs predicted by all families of atmosphere codes fairly repre- 
sented the ionizing $\mathrm{OB}$ stars for energies $<35 \mathrm{eV}$. In contrast to the behavior at lower ionization potential, we started to see differences between the atmosphere models and the observations for [NeIII]. For WM-basic, TLUSTY, and SNC02, we saw significant scatter between the observations and the model predictions, with the predicted line strengths ranging from over- to under-predicting [NeIII] by $\sim 70 \%$. TLUSTY, which is a plane-parallel model, produced model HiI regions that consistently had less [NeIII] and more [OII] than the other atmosphere models. This behavior is consistent with the softer SEDs of plane-parallel models (Morisset et al., 2004). Meanwhile, the CLOUDY models ionized by CoStar model atmospheres, which have the hardest SEDs, consistently over-predicted [NeIII] by greater than a factor of two. The discrepancies at these higher ionization potential lines may reflect variations between the treatment of winds and the actual winds in the stars (Gabler et al., 1989). This scatter between the predictions and the observations should be considered when using these lines as diagnostics. Taking into account both low and high ions we found the WM-basic atmosphere models, with detailed treatment of both wind- and lineblanketing, best represent the ionizing stars.

Finally, we compared the effective temperatures derived from our nebular modeling to those expected from SpT- $T_{\text {eff }}$ calibrations in the literature. Literature calibrations determine $T_{\text {eff }}$ by matching photospheric absorption lines in model SEDs to those of high resolution stellar spectra. In contrast, nebular modeling would be additionally sensitive to any changes to the SED from the outer layers of the atmosphere above the photosphere. Our $T_{\text {eff }}$ are broadly consistent with those expected from literature calibrations. However, the uncertainty in $\log (g)$ in our sample translates to uncertainty in $T_{\text {eff }}$ of up to a few thousand degrees. To fully capitalize on this diagnostic requires a sample with higher resolution stellar spectra.

In this thesis we used two under-utilized methods to investigate radiative feedback from massive stars. Using ionization parameter mapping, we discovered optically thin ionization cones in two starburst galaxies. This unprecedented view of the morphology of escaping radiation suggests that an orientation bias contributes to the low detection rate of escaping Lyman continuum. An ionization parameter mapping 
study, with more local starbursts, would further establish which galactic properties and processes contribute to the formation of these cones, and the escape of ionizing radiation. Another avenue is to model the expected distribution of Lyman continuum detections, assuming random orientation and a small opening angle for escape. This modeled distribution could be compared to the statistics from recent narrowband imaging campaigns, such as that of Iwata et al. (2009); Nestor et al. (2011).

The second technique we used involved photoionization simulations of single-star HiI regions. Even though we used general grids of stellar atmospheres for the ionizing sources, our method clearly distinguished between the shapes of massive-star SEDs predicted by different atmosphere models. Future work using a larger sample that includes stars with $\mathrm{SpT}$ earlier that $\mathrm{O} 5.5 \mathrm{~V}$ would improve on the groundwork laid by this study. Another valuable avenue would be to expand the sample to other metallicities, since lower metallicity stars have weaker winds. If the treatment of winds causes the discrepancy at [NeIII], then one might expect the scatter to be lower in a sample of stars with lower metallicity. In fact, Lamb et al. (in prep) has already identified a complete sample of field OB stars in the SMC from which a sample could be drawn. Another logical next step would be to tailor fit the stellar atmosphere models to the ionizing stars (e.g., Simón-Díaz et al., 2011), and use those SEDs as the ionizing source in the photoionization simulations. 


\section{BIBLIOGRAPHY}

Alcock, C., et al. 1997, AJ, 114, 326

Allen, D. A., Wright, A. E., \& Goss, W. M. 1976, MNRAS, 177, 91

Allen, M. G., Groves, B. A., Dopita, M. A., Sutherland, R. S., \& Kewley, L. J. 2008, ApJS, 178, 20

Alonso-Herrero, A., Takagi, T., Baker, A. J., Rieke, G. H., Rieke, M. J., Imanishi, M., \& Scoville, N. Z. 2004, ApJ, 612, 222

Alton, P. B., Draper, P. W., Gledhill, T. M., Stockdale, D. P., Scarrott, S. M., \& Wolstencroft, R. D. 1994, MNRAS, 270, 238

Annibali, F., Greggio, L., Tosi, M., Aloisi, A., \& Leitherer, C. 2003, AJ, 126, 2752

Annibali, F., Tosi, M., Monelli, M., Sirianni, M., Montegriffo, P., Aloisi, A., \& Greggio, L. 2009, AJ, 138, 169

Baldwin, J. A., Phillips, M. M., \& Terlevich, R. 1981, PASP, 93, 5

Beck, S. C., Turner, J. L., Ho, P. T. P., Lacy, J. H., \& Kelly, D. M. 1996, ApJ, 457, 610

Bell, E. F., McIntosh, D. H., Katz, N., \& Weinberg, M. D. 2003, ApJS, 149, 289

Benjamin, R. A., Skillman, E. D., \& Smits, D. P. 2002, ApJ, 569, 288

Benson, A., Venkatesan, A., \& Shull, J. M. 2012, ArXiv e-prints

Bergvall, N., Leitet, E., Zackrisson, E., \& Marquart, T. 2013, ArXiv e-prints

Bouwens, R. J., et al. 2012, ApJ, 752, L5

Bouwens, R. J., et al. 2010, ApJ, 708, L69

Bresolin, F., Kennicutt, R. C., \& Garnett, D. R. 1999, ApJ, 510, 104

Bridge, C. R., et al. 2010, ApJ, 720, 465

Burstein, D. \& Heiles, C. 1982, AJ, 87, 1165

Caldwell, N. \& Phillips, M. M. 1989, ApJ, 338, 789 
Calzetti, D., Conselice, C. J., Gallagher, J. S., \& Kinney, A. L. 1999, AJ, 118, 797

Calzetti, D., Meurer, G. R., Bohlin, R. C., Garnett, D. R., Kinney, A. L., Leitherer, C., \& Storchi-Bergmann, T. 1997, AJ, 114, 1834

Calzetti, D., et al. 2010, ApJ, 714, 1256

Cardelli, J. A., Clayton, G. C., \& Mathis, J. S. 1989, ApJ, 345, 245

Chandar, R., Leitherer, C., Tremonti, C. A., Calzetti, D., Aloisi, A., Meurer, G. R., \& de Mello, D. 2005, ApJ, 628, 210

Ciardi, B., Bianchi, S., \& Ferrara, A. 2002, MNRAS, 331, 463

Clarke, C. \& Oey, M. S. 2002, MNRAS, 337, 1299

Conti, P. S. 1973, ApJ, 179, 181

Copetti, M. V. F., Pastoriza, M. G., \& Dottori, H. A. 1986, A\&A, 156, 111

Cowie, L. L., Barger, A. J., \& Trouille, L. 2009, ApJ, 692, 1476

Cresci, G., Vanzi, L., \& Sauvage, M. 2005, A\&A, 433, 447

Crowther, P. A., Lennon, D. J., \& Walborn, N. R. 2006, A\&A, 446, 279

Crowther, P. A., Pasquali, A., De Marco, O., Schmutz, W., Hillier, D. J., \& de Koter, A. 1999, A\&A, 350, 1007

Davies, R. D., Elliott, K. H., \& Meaburn, J. 1976, MmRAS, 81, 89

de Vaucouleurs, G., de Vaucouleurs, A., Corwin, H. G., Buta, R. J., Paturel, G., \& Fouqué, P. 1991, Third Reference Catalogue of Bright Galaxies. Volume I: Explanations and references. Volume II: Data for galaxies between $0^{h}$ and $12^{h}$. Volume III: Data for galaxies between $12^{h}$ and $24^{h}$.

Dors, O. L. \& Copetti, M. V. F. 2003, A\&A, 404, 969

Dove, J. B., Shull, J. M., \& Ferrara, A. 2000, ApJ, 531, 846

Dufour, R. J. 1975, ApJ, 195, 315

Edmunds, M. G. \& Pagel, B. E. J. 1984, MNRAS, 211, 507

Elson, E. C., de Blok, W. J. G., \& Kraan-Korteweg, R. C. 2013, MNRAS, 429, 2550

Ercolano, B., Bastian, N., \& Stasińska, G. 2007, MNRAS, 379, 945

Esteban, C., Smith, L. J., Vilchez, J. M., \& Clegg, R. E. S. 1993, A\&A, 272, 299

Fabbiano, G., Kim, D.-W., \& Trinchieri, G. 1992, ApJS, 80, 531 
Fan, X., Carilli, C. L., \& Keating, B. 2006, ARA\&A, 44, 415

Fan, X., et al. 2001, AJ, 122, 2833

Ferland, G. J., Korista, K. T., Verner, D. A., Ferguson, J. W., Kingdon, J. B., \& Verner, E. M. 1998, PASP, 110, 761

Fernandez, E. R. \& Shull, J. M. 2011, ApJ, 731, 20

Fujita, A., Martin, C. L., Mac Low, M.-M., \& Abel, T. 2003, ApJ, 599, 50

Gabler, R., Gabler, A., Kudritzki, R. P., Puls, J., \& Pauldrach, A. 1989, A\&A, 226, 162

Garnett, D. R. 1999, in IAU Symposium, Vol. 190, New Views of the Magellanic Clouds, ed. Y.-H. Chu, N. Suntzeff, J. Hesser, \& D. Bohlender, 266

Giammanco, C., Beckman, J. E., Zurita, A., \& Relaño, M. 2004, A\&A, 424, 877

Gil de Paz, A., Madore, B. F., \& Pevunova, O. 2003, ApJS, 147, 29

Giveon, U., Sternberg, A., Lutz, D., Feuchtgruber, H., \& Pauldrach, A. W. A. 2002, ApJ, 566, 880

Gnedin, N. Y., Kravtsov, A. V., \& Chen, H.-W. 2008, ApJ, 672, 765

Gonzalez-Delgado, R. M., et al. 1994, ApJ, 437, 239

Gordon, K. D., Clayton, G. C., Misselt, K. A., Landolt, A. U., \& Wolff, M. J. 2003, ApJ, 594, 279

Gorjian, V., Turner, J. L., \& Beck, S. C. 2001, ApJ, 554, L29

Graczyk, D., Soszyński, I., Poleski, R., Pietrzyński, G., Udalski, A., Szymański, M. K., Kubiak, M., Wyrzykowski, Ł., \& Ulaczyk, K. 2011, AcA, 61, 103

Grimes, J. P., Heckman, T., Aloisi, A., Calzetti, D., Leitherer, C., Martin, C. L., Meurer, G., Sembach, K., \& Strickland, D. 2009, ApJS, 181, 272

Hadfield, L. J. \& Crowther, P. A. 2006, MNRAS, 368, 1822

Haffner, L. M., Reynolds, R. J., \& Tufte, S. L. 1999, ApJ, 523, 223

Hameed, S. \& Devereux, N. 1999, AJ, 118, 730

Hamuy, M., Suntzeff, N. B., Heathcote, S. R., Walker, A. R., Gigoux, P., \& Phillips, M. M. 1994, PASP, 106, 566

Heckman, T. M., Armus, L., \& Miley, G. K. 1990, ApJS, 74, 833

Heckman, T. M., et al. 2011, ApJ, 730, 5 
Heckman, T. M. \& Leitherer, C. 1997, AJ, 114, 69

Heckman, T. M., Sembach, K. R., Meurer, G. R., Leitherer, C., Calzetti, D., \& Martin, C. L. 2001, ApJ, 558, 56

Henize, K. G. 1956, ApJS, 2, 315

Herrero, A., Corral, L. J., Villamariz, M. R., \& Martín, E. L. 1999, A\&A, 348, 542

Ho, L. C. \& Filippenko, A. V. 1996, ApJ, 472, 600

Ho, L. C., Li, Z.-Y., Barth, A. J., Seigar, M. S., \& Peng, C. Y. 2011, ApJS, 197, 21

Hota, A. \& Saikia, D. J. 2005, MNRAS, 356, 998

Hubeny, I. \& Lanz, T. 1995, ApJ, 439, 875

Hunt, L. K. \& Hirashita, H. 2009, A\&A, 507, 1327

Hunter, D. A. \& Massey, P. 1990, AJ, 99, 846

Iwata, I., et al. 2009, ApJ, 692, 1287

Jaskot, A. E. \& Oey, M. S. 2013, ApJ, 766, 91

Johnson, K. E. \& Kobulnicky, H. A. 2003, ApJ, 597, 923

Johnson, K. E., Leitherer, C., Vacca, W. D., \& Conti, P. S. 2000, AJ, 120, 1273

Kaler, J. B. 1978, ApJ, 220, 887

Kennicutt, R. C., et al. 2011, PASP, 123, 1347

Kennicutt, R. C. 1983, ApJ, 272, 54

Kennicutt, R. C. 1984, ApJ, 287, 116

Kennicutt, R. C., Bresolin, F., French, H., \& Martin, P. 2000, ApJ, 537, 589

Kennicutt, R. C., Hao, C.-N., Calzetti, D., Moustakas, J., Dale, D. A., Bendo, G., Engelbracht, C. W., Johnson, B. D., \& Lee, J. C. 2009, ApJ, 703, 1672

Kennicutt, R. C., Lee, J. C., Funes, S. J., Sakai, S., \& Akiyama, S. 2008, ApJS, 178, 247

Kewley, L. J. \& Dopita, M. A. 2002, ApJS, 142, 35

Kim, J.-h., Krumholz, M. R., Wise, J. H., Turk, M. J., Goldbaum, N. J., \& Abel, T. 2012, ArXiv e-prints

Kiminki, D. C. \& Kobulnicky, H. A. 2012, ApJ, 751, 4 
Kingdon, J. B. \& Ferland, G. J. 1995, ApJ, 450, 691

Kobulnicky, H. A., Dickey, J. M., Sargent, A. I., Hogg, D. E., \& Conti, P. S. 1995, AJ, 110, 116

Kobulnicky, H. A. \& Skillman, E. D. 2008, AJ, 135, 527

Koribalski, B. S., et al. 2004, AJ, 128, 16

Kreimeyer, K. \& Veilleux, S. 2013, ApJ, 772, L11

Kudritzki, R. P. \& Hummer, D. G. 1990, ARA\&A, 28, 303

Kudritzki, R.-P. \& Puls, J. 2000, ARA\&A, 38, 613

Lamers, H. J. G. L. M., Snow, T. P., \& Lindholm, D. M. 1995, ApJ, 455, 269

Lanz, T. \& Hubeny, I. 2003, ApJS, 146, 417

Lee, H. \& Skillman, E. D. 2004, ApJ, 614, 698

Leitet, E., Bergvall, N., Hayes, M., Linné, S., \& Zackrisson, E. 2013, A\&A, 553, A106

Leitet, E., Bergvall, N., Piskunov, N., \& Andersson, B.-G. 2011, A\&A, 532, A107

Leitherer, C., Ferguson, H. C., Heckman, T. M., \& Lowenthal, J. D. 1995, ApJ, 454, L19

Leitherer, C., Schaerer, D., Goldader, J. D., González Delgado, R. M., Robert, C., Kune, D. F., de Mello, D. F., Devost, D., \& Heckman, T. M. 1999, ApJS, 123, 3

Liu, X.-W. \& Danziger, J. 1993, MNRAS, 263, 256

López-Sánchez, Á. R., Koribalski, B. S., van Eymeren, J., Esteban, C., Kirby, E., Jerjen, H., \& Lonsdale, N. 2012, MNRAS, 419, 1051

Mac Low, M.-M. \& McCray, R. 1988, ApJ, 324, 776

Madau, P., Haardt, F., \& Rees, M. J. 1999, ApJ, 514, 648

Marlowe, A. T., Heckman, T. M., Wyse, R. F. G., \& Schommer, R. 1995, ApJ, 438, 563

Martin, C. L. \& Kennicutt, R. C. 1995, ApJ, 447, 171

Martín-Hernández, N. L., Vermeij, R., Tielens, A. G. G. M., van der Hulst, J. M., \& Peeters, E. 2002, A\&A, 389, 286

Martins, F., Schaerer, D., \& Hillier, D. J. 2005, A\&A, 436, 1049

Massey, P. 2002, ApJS, 141, 81 
Massey, P., Puls, J., Pauldrach, A. W. A., Bresolin, F., Kudritzki, R. P., \& Simon, T. 2005, ApJ, 627, 477

Mathis, J. S., Chu, Y.-H., \& Peterson, D. E. 1985, ApJ, 292, 155

McGaugh, S. S. 1991, ApJ, 380, 140

Meier, D. S., Turner, J. L., \& Beck, S. C. 2002, AJ, 124, 877

Meiksin, A. 2005, MNRAS, 356, 596

Melnick, J., Moles, M., \& Terlevich, R. 1985, A\&A, 149, L24

Méndez, D. I., Esteban, C., Filipović , M. D., Ehle, M., Haberl, F., Pietsch, W., \& Haynes, R. F. 1999, A\&A, 349, 801

Meurer, G. R., Freeman, K. C., Dopita, M. A., \& Cacciari, C. 1992, AJ, 103, 60

Meurer, G. R., et al. 2006, ApJS, 165, 307

Meurer, G. R., Staveley-Smith, L., \& Killeen, N. E. B. 1998, MNRAS, 300, 705

Mokiem, M. R., et al. 2007, A\&A, 465, 1003

Mokiem, M. R., Martín-Hernández, N. L., Lenorzer, A., de Koter, A., \& Tielens, A. G. G. M. 2004, A\&A, 419, 319

Morisset, C., Schaerer, D., Bouret, J.-C., \& Martins, F. 2004, A\&A, 415, 577

Morisset, C., Schaerer, D., Martín-Hernández, N. L., Peeters, E., Damour, F., Baluteau, J.-P., Cox, P., \& Roelfsema, P. 2002, A\&A, 386, 558

Morisset, C., Stasińska, G., \& Peña, M. 2005, MNRAS, 360, 499

Moustakas, J., Kennicutt, R. C., Tremonti, C. A., Dale, D. A., Smith, J.-D. T., \& Calzetti, D. 2010, ApJS, 190, 233

Murray, N., Ménard, B., \& Thompson, T. A. 2011, ApJ, 735, 66

Nestor, D. B., Shapley, A. E., Steidel, C. C., \& Siana, B. 2011, ApJ, 736, 18

Nordgren, T. E., Chengalur, J. N., Salpeter, E. E., \& Terzian, Y. 1997, AJ, 114, 913

O’Connell, R. W., Gallagher, J. S., \& Hunter, D. A. 1994, ApJ, 433, 65

Oey, M. S., Dopita, M. A., Shields, J. C., \& Smith, R. C. 2000, ApJS, 128, 511

Oey, M. S., et al. 2007, ApJ, 661, 801

Omar, A. \& Dwarakanath, K. S. 2005, Journal of Astrophysics and Astronomy, 26, 1

Osterbrock, D. \& Flather, E. 1959, ApJ, 129, 26 
Osterbrock, D. E. \& Ferland, G. J. 2006, Astrophysics of gaseous nebulae and active galactic nuclei

Ott, J., Walter, F., \& Brinks, E. 2005, MNRAS, 358, 1423

Paardekooper, J.-P., Pelupessy, F. I., Altay, G., \& Kruip, C. J. H. 2011, A\&A, 530, A 87

Pauldrach, A. W. A., Hoffmann, T. L., \& Lennon, M. 2001, A\&A, 375, 161

Pauldrach, A. W. A., Lennon, M., Hoffmann, T. L., Sellmaier, F., Kudritzki, R.-P., \& Puls, J. 1998, in Astronomical Society of the Pacific Conference Series, Vol. 131, Properties of Hot Luminous Stars, ed. I. Howarth, 258

Peimbert, M. 1967, ApJ, 150, 825

Pellegrini, E. W., Baldwin, J. A., \& Ferland, G. J. 2011, ApJ, 738, 34

Pellegrini, E. W., Oey, M. S., Winkler, P. F., Points, S. D., Smith, R. C., Jaskot, A. E., \& Zastrow, J. 2012, ApJ, 755, 40

Pérez-Montero, E. \& Díaz, A. I. 2005, MNRAS, 361, 1063

Pogge, R. W. 1988, ApJ, 328, 519

Prinja, R. K., Barlow, M. J., \& Howarth, I. D. 1990, ApJ, 361, 607

Raimann, D., Bica, E., Storchi-Bergmann, T., Melnick, J., \& Schmitt, H. 2000, MNRAS, 314, 295

Rand, R. J. 1998, ApJ, 501, 137

Razoumov, A. O. \& Sommer-Larsen, J. 2010, ApJ, 710, 1239

Reines, A. E., Sivakoff, G. R., Johnson, K. E., \& Brogan, C. L. 2011, Nature, 470, 66

Rigby, J. R. \& Rieke, G. H. 2004, ApJ, 606, 237

Rubin, R. H. 1989, ApJS, 69, 897

Rubin, R. H., Simpson, J. P., Haas, M. R., \& Erickson, E. F. 1991, ApJ, 374, 564

Sakai, S., Ferrarese, L., Kennicutt, R. C., \& Saha, A. 2004, ApJ, 608, 42

Sana, H., Gosset, E., \& Evans, C. J. 2009, MNRAS, 400, 1479

Sana, H., James, G., \& Gosset, E. 2011, MNRAS, 416, 817

Sauvage, M., Thuan, T. X., \& Lagage, P. O. 1997, A\&A, 325, 98

Schaerer, D., Contini, T., \& Pindao, M. 1999, A\&AS, 136, 35 
Schaerer, D. \& de Koter, A. 1997, A\&A, 322, 598

Schaerer, D., Meynet, G., Maeder, A., \& Schaller, G. 1993, A\&AS, 98, 523

Schaerer, D. \& Schmutz, W. 1994, A\&A, 288, 231

Sellmaier, F. H., Yamamoto, T., Pauldrach, A. W. A., \& Rubin, R. H. 1996, A\&A, 305, L37

Shankar, F. \& Mathur, S. 2007, ApJ, 660, 1051

Shapley, A. E., Steidel, C. C., Pettini, M., Adelberger, K. L., \& Erb, D. K. 2006, ApJ, 651, 688

Sharp, R. G. \& Bland-Hawthorn, J. 2010, ApJ, 711, 818

Shopbell, P. L. \& Bland-Hawthorn, J. 1998, ApJ, 493, 129

Shull, J. M., Harness, A., Trenti, M., \& Smith, B. D. 2012, ApJ, 747, 100

Siana, B., et al. 2007, ApJ, 668, 62

Siana, B., et al. 2010, ApJ, 723, 241

Simón-Díaz, S., García-Rojas, J., Esteban, C., Stasińska, G., López-Sánchez, A. R., \& Morisset, C. 2011, A\&A, 530, A57

Simón-Díaz, S. \& Stasińska, G. 2008, MNRAS, 389, 1009

Simpson, J. P., Colgan, S. W. J., Rubin, R. H., Erickson, E. F., \& Haas, M. R. 1995, ApJ, 444, 721

Simpson, J. P., Rubin, R. H., Erickson, E. F., \& Haas, M. R. 1986, ApJ, 311, 895

Skrutskie, M. F., et al. 2003, VizieR Online Data Catalog, 7233, 0

Smith, B. J., Struck, C., Appleton, P. N., Charmandaris, V., \& Reach, W. 2005, in Bulletin of the American Astronomical Society, Vol. 37, American Astronomical Society Meeting Abstracts \#206, 449

Smith, L. J., Norris, R. P. F., \& Crowther, P. A. 2002, MNRAS, 337, 1309

Stasińska, G. \& Leitherer, C. 1996, ApJS, 107, 661

Stasińska, G. \& Schaerer, D. 1997, A\&A, 322, 615

Steidel, C. C., Pettini, M., \& Adelberger, K. L. 2001, ApJ, 546, 665

Storchi-Bergmann, T., Calzetti, D., \& Kinney, A. L. 1994, ApJ, 429, 572

Stoy, R. H. 1933, MNRAS, 93, 588 
Strickland, D. K., Heckman, T. M., Colbert, E. J. M., Hoopes, C. G., \& Weaver, K. A. 2004, ApJS, 151, 193

Summers, L. K., Stevens, I. R., Strickland, D. K., \& Heckman, T. M. 2004, MNRAS, 351,1

Taylor, V. A., Jansen, R. A., Windhorst, R. A., Odewahn, S. C., \& Hibbard, J. E. 2005, ApJ, 630, 784

Tosi, M., Sabbi, E., Bellazzini, M., Aloisi, A., Greggio, L., Leitherer, C., \& Montegriffo, P. 2001, AJ, 122, 1271

Trundle, C., Lennon, D. J., Puls, J., \& Dufton, P. L. 2004, A\&A, 417, 217

Tsamis, Y. G. \& Péquignot, D. 2005, MNRAS, 364, 687

Turner, J. L. \& Beck, S. C. 2004, ApJ, 602, L85

Turner, J. L., Beck, S. C., Crosthwaite, L. P., Larkin, J. E., McLean, I. S., \& Meier, D. S. 2003, Nature, 423, 621

Turner, J. L., Beck, S. C., \& Ho, P. T. P. 2000, ApJ, 532, L109

Turner, J. L., Ho, P. T. P., \& Beck, S. C. 1998, AJ, 116, 1212

Udalski, A., Soszynski, I., Szymanski, M. K., Kubiak, M., Pietrzynski, G., Wyrzykowski, L., Szewczyk, O., Ulaczyk, K., \& Poleski, R. 2008, AcA, 58, 89

Vacca, W. D. \& Conti, P. S. 1992, ApJ, 401, 543

Vacca, W. D., Garmany, C. D., \& Shull, J. M. 1996, ApJ, 460, 914

Vagshette, N. D., Pandge, M. B., Pandey, S. K., \& Patil, M. K. 2012, NewA, 17, 524

van Hoof, P. A. M., Weingartner, J. C., Martin, P. G., Volk, K., \& Ferland, G. J. 2004, in Astronomical Society of the Pacific Conference Series, Vol. 313, Asymmetrical Planetary Nebulae III: Winds, Structure and the Thunderbird, ed. M. Meixner, J. H. Kastner, B. Balick, \& N. Soker, 380

Vanzella, E., et al. 2010, ApJ, 725, 1011

Vanzella, E., et al. 2012, ApJ, 751, 70

Vanzi, L., Cresci, G., Sauvage, M., \& Thompson, R. 2011, A\&A, 534, A70

Veilleux, S., Cecil, G., \& Bland-Hawthorn, J. 2005, ARA\&A, 43, 769

Veilleux, S. \& Rupke, D. S. 2002, ApJ, 565, L63

Veilleux, S., Shopbell, P. L., Rupke, D. S., Bland-Hawthorn, J., \& Cecil, G. 2003, AJ, 126, 2185 
Veilleux, S., et al. 2010, AJ, 139, 145

Viegas, S. M. \& Clegg, R. E. S. 1994, MNRAS, 271, 993

Vilchez, J. M. \& Pagel, B. E. J. 1988, MNRAS, 231, 257

Voges, E. S., Oey, M. S., Walterbos, R. A. M., \& Wilkinson, T. M. 2008, AJ, 135, 1291

Walborn, N. R. \& Fitzpatrick, E. L. 1990, PASP, 102, 379

Walsh, J. R. \& Roy, J.-R. 1989, MNRAS, 239, 297

Weingartner, J. C. \& Draine, B. T. 2001, ApJ, 548, 296

Westera, P., Cuisinier, F., Telles, E., \& Kehrig, C. 2004, A\&A, 423, 133

Westerlund, B. E. 1997, The Magellanic Clouds

Williams, R. E. 1992, ApJ, 392, 99

Wise, J. H. \& Cen, R. 2009, ApJ, 693, 984

Yajima, H., Choi, J.-H., \& Nagamine, K. 2011, MNRAS, 412, 411

Yajima, H., Li, Y., Zhu, Q., Abel, T., Gronwall, C., \& Ciardullo, R. 2012, ArXiv e-prints

Zanstra, H. 1927, ApJ, 65, 50

Zastrow, J., Oey, M. S., \& Pellegrini, E. W. 2013, ApJ, 769, 94

Zastrow, J., Oey, M. S., Veilleux, S., McDonald, M., \& Martin, C. L. 2011, ApJ, 741, L17 